\title{
NAGATA COMPACTIFICATION FOR ALGEBRAIC SPACES
}

\author{
BRIAN CONRAD, MAX LIEBLICH, AND MARTIN OLSSON
}

\begin{abstract}
We prove the Nagata compactification theorem for any separated map of finite type between quasi-compact and quasi-separated algebraic spaces, generalizing earlier results of Raoult. Along the way we also prove (and use) absolute noetherian approximation for such algebraic spaces, generalizing earlier results in the case of schemes.
\end{abstract}

To the memory of Masayoshi Nagata

\section{INTRODUCTION}

1.1. Motivation. The Nagata compactification theorem for schemes is a very useful and fundamental result. It says that if $S$ is a quasi-compact and quasi-separated scheme (e.g., any noetherian scheme) and if $f: X \rightarrow S$ is a separated map of finite type from a scheme $X$ then $f$ fits into a commutative diagram of schemes

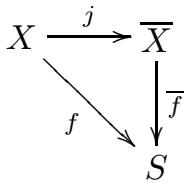

with $j$ an open immersion and $\bar{f}$ proper; we call such an $\bar{X}$ an $S$-compactification of $X$.

Nagata's papers ([N1], [N2]) focused on the case of noetherian schemes and unfortunately are difficult to read nowadays (due to the use of an older style of algebraic geometry), but there are several available proofs in modern language. The proof by Lütkebohmert $[\mathrm{L}$ applies in the noetherian case, and the proof of Deligne $([\mathrm{D}, \mathrm{C} 2])$ is a modern interpretation of Nagata's method which applies in the general scheme case. The preprint [Vo] by Vojta gives an exposition of Deligne's approach in the noetherian case. Temkin has recently introduced some new valuation-theoretic ideas that give yet another proof in the general scheme case. The noetherian case is the essential one for proving the theorem because it implies the general case via approximation arguments $[\mathrm{C} 2$, Thm. 4.3].

An important application of the Nagata compactification theorem for schemes is in the definition of étale cohomology with proper supports for any separated map of finite type $f: X \rightarrow S$ between arbitrary schemes. Since any algebraic space is étale-locally a scheme, the main obstacle to having a similar construction of such a theory for étale cohomology of algebraic spaces is the availability of a version of Nagata's theorem for algebraic spaces. Strictly speaking, it is possible to develop the full "six operations" formalism even for nonseparated Artin stacks ([LO1, [LO2]) despite the lack of a compactification theorem in such cases. However, the availability of a form of Nagata's theorem simplifies matters tremendously, and there are cohomological applications for which the approach through compactifications seems essential, such as the proof of Fujiwara's theorem for algebraic spaces [Va] (from which one can deduce the result for Deligne-Mumford stacks via the

Date: October 25, 2010.

1991 Mathematics Subject Classification. Primary 14A15; Secondary 14E99.

Key words and phrases. compactification, blow-up.

BC was partially supported by NSF grant DMS-0917686, ML was partially supported by NSF grant DMS-0758391 and the Sloan Foundation, and MO was partially supported by NSF grants DMS-0714086 and DMS-0748718 and the Sloan Foundation. The authors are grateful to the Mathematical Institute at Oberwolfach and MSRI for their hospitality and atmosphere, and to Johan de Jong and especially Ofer Gabber for helpful suggestions at the start of this project. We also received numerous helpful comments from David Rydh on an early draft of this paper. We are also grateful to Rydh for obtaining and forwarding a copy of the unpublished thesis of Raoult [R3], after the first version of this paper was written. 
use of coarse spaces). The existence of compactifications is useful in many non-cohomological contexts as well.

1.2. Main results. In his (unpublished) thesis [R3], Raoult proved the Nagata compactification theorem in the following situations:

(1) $X$ is a normal algebraic space and $S$ is noetherian [R3, Proposition, page 2];

(2) $X$ is arbitrary and $S$ is the spectrum of $\mathbf{Z}$, a field $k$, or a complete local noetherian ring [R3, Theorem, page 11].

A sketch of the second case when $S$ is the spectrum of a field is contained in [R2]. One can deduce from the second case that compactifications exist when $S$ is separated and of finite type over $\mathbf{Z}$, a field, or a complete local noetherian ring. Raoult's argument was conditional on an unpublished result of Deligne concerning the existence and properties of quotients by finite group actions on separated algebraic spaces. This result of Deligne is a consequence of subsequent general results on the existence of coarse moduli spaces associated to Artin stacks, which we shall review later (and it is also addressed in [Ry1, Thm. 5.4] in a stronger form).

Following a sketch suggested to us by Gabber, we handle an essentially arbitrary base $S$ :

Theorem 1.2.1. Let $f: X \rightarrow S$ be a separated map of finite type between algebraic spaces, with $S$ quasicompact and quasi-separated. There exists an open immersion $j: X \hookrightarrow \bar{X}$ over $S$ into an algebraic space $\bar{X}$ that is proper over $S$. If $f$ is finitely presented then $\bar{X}$ may be taken to be finitely presented over $S$.

The proof of Theorem 1.2.1 consists of two rather separate parts: technical approximation arguments reduce the proof to the case when $S$ is of finite presentation over $\operatorname{Spec} \mathbf{Z}$ (or an excellent noetherian scheme), and geometric arguments handle this special case by reducing to the known normal case. (We include a proof in the normal case, so there is no logical dependence on the unpublished [R3].) We became aware of Raoult's work only after working out our proof in general, and our basic strategy (after reducing to $S$ of finite presentation over $\mathbf{Z}$ ) is similar to Raoult's. The reader who is only interested in the case that $S$ is of finite presentation over $\mathbf{Z}$ or a field (or more generally, an excellent noetherian scheme) may go immediately to $\$ 2$ and can ignore the rest of this paper. Theorem 1.2.1 has also been announced by K. Fujiwara and F. Kato, to appear in a book in progress, as well as by D. Rydh (who has also announced progress in the case of Deligne-Mumford stacks).

The approximation part of our proof contains some results which are useful for eliminating noetherian hypotheses more generally, so we now make some remarks on this feature of the present work. Limit methods of Grothendieck [EGA, $\left.\mathrm{IV}_{3}, \S 8-\S 11\right]$ are a standard technique for avoiding noetherian hypotheses, and a very useful supplement to these methods is the remarkable [TT, App. C]. The key innovation there going beyond [EGA is an absolute noetherian approximation property [TT, Thm. C.9]: any quasi-compact and quasiseparated scheme $S$ admits the form $S \simeq \lim S_{\lambda}$ where $\left\{S_{\lambda}\right\}$ is an inverse system of $\mathbf{Z}$-schemes of finite type, with affine transition maps $S_{\lambda^{\prime}} \rightarrow S_{\lambda}$ for $\lambda^{\prime} \geq \lambda$. (Conversely, any such limit scheme is obviously affine over any $S_{\lambda_{0}}$ and so is necessarily quasi-compact and quasi-separated.) The crux of the matter is that every quasi-compact and quasi-separated scheme $S$ is affine over a $\mathbf{Z}$-scheme of finite type. This appromxation result was used to avoid noetherian hypotheses in Nagata's theorem for schemes in [C2, and we likewise need a version of it for algebraic spaces. This is of interest in its own right, so we state it here (and prove it in 93 . We note that D. Rydh has a different proof of this result that also works for certain algebraic stacks Ry2.

Theorem 1.2.2. Let $S$ be a quasi-compact and quasi-separated algebraic space. There exists an inverse system $\left\{S_{\lambda}\right\}$ of algebraic spaces of finite presentation over $\mathbf{Z}$ such that the transition maps $S_{\lambda^{\prime}} \rightarrow S_{\lambda}$ are affine for $\lambda^{\prime} \geq \lambda$ and $S \simeq \lim _{\longleftrightarrow} S_{\lambda}$. Moreover, $S$ is separated if and only if $S_{\lambda}$ is separated for sufficiently large $\lambda$.

Remark 1.2.3. The limit algebraic space $\lim S_{\lambda}$ is defined étale-locally over any single $S_{\lambda_{0}}$ by using the analogous well-known limit construction in the case of schemes. By working étale-locally it is easy to check that such an algebraic space has the universal property of an inverse limit in the category of algebraic spaces. Also, since $\lim _{\longleftarrow} S_{\lambda}$ is affine over any $S_{\lambda_{0}}$, it is quasi-compact and quasi-separated. 
We now briefly outline the paper. We first consider Theorem 1.2.1 when $S$ is of finite presentation over an excellent noetherian scheme ( $\operatorname{such}$ as $\operatorname{Spec} \mathbf{Z}$ ). This case is the focus of our efforts in $₫ 2$, and the base $S$ is fixed throughout most this section but we progressively simplify $X$. In 2.1 we use the cotangent complex to reduce to the case when $X$ is reduced. Then in $\$ 2.2$ we use a contraction result of Artin [A, Thm. 6.1] and various results of Raynaud-Gruson [RG, I, §5.7] to reduce to the case when $X$ is normal. The case of normal $X$ is handled in $\$ 2.4$ by using a group quotient argument to reduce to the known case (whose proof we also provide, for the convenience of the reader) when $X$ is normal and $S$ is an excellent noetherian scheme. Note that this settles Theorem 1.2.1 for all "practical" cases, but not yet the general noetherian case.

The passage to the general case of Theorem 1.2 .1 is the aim of 93 , via various approximation methods. In $\$ 3.1$ we use stratification techniques of Raynaud-Gruson for algebraic spaces to prove Theorem 1.2 .2 by reducing it to the known case of schemes, and then in $\$ 3.2$ we reduce to the proof of Theorem 1.2.1 in general to the case when $f: X \rightarrow S$ is finitely presented (not just finite type). An application of Theorem 1.2.2 then allows us to reduce Theorem 1.2.1 to the proved case when $S$ is of finite presentation over $\mathbf{Z}$. It is only at this point that the general noetherian case is also settled.

The appendices provide some foundational facts we need concerning algebraic spaces; in 11.3 we offer some "justification" for the appendices. Much of what is in the appendices may be known to some experts, but we did not know of a reference in the literature for the results discussed there. The reader who is content with taking $S$ to be finitely presented over an excellent scheme in Theorem 1.2.1 can ignore $\$ 3$ and almost all of the appendices, and other readers should probably only consult the appendices when they are cited in the main text.

New ideas are needed to prove a general version of Nagata's theorem for Deligne-Mumford stacks.

1.3. Terminology and conventions. We write qcqs as shorthand for "quasi-compact and quasi-separated" (for schemes, algebraic spaces, or morphisms between them), and we freely identify any scheme with the corresponding sheaf of sets that it represents on the étale site of the category of schemes.

The reader who wishes to understand the proof of Theorem 1.2.1 for general noetherian $S$ (or anything beyond the case of $S$ of finite presentation over an excellent noetherian scheme) will need to read 93 , for which the following comments should be helpful. Although quasi-separatedness is required in the definition of an algebraic space in $[\mathrm{K}$, there are natural reasons for wanting to avoid such foundational restrictions. We need to use several kinds of pushout and gluing constructions with algebraic spaces, and the construction and study of these pushouts becomes unpleasant if we cannot postpone the consideration of quasi-separatedness properties until after the construction has been carried out. It is a remarkable fact that quasi-separatedness is not necessary in the foundations of the theory of algebraic spaces; this was known to some experts long ago, but seems to not be as widely known as it should be.

We define an algebraic space $X$ to be an algebraic space over Spec $\mathbf{Z}$ as in [RG, I, 5.7.1]: it is an étale sheaf on the category of schemes such that it is isomorphic to a quotient sheaf $U / R$ for an étale equivalence relation in schemes $R \rightrightarrows U$; there are no quasi-compactness hypotheses in this definition. The key point is that by using the method of proof of [RG, I, 5.7.2], it can be proved that for any such $X=U / R$, the fiber product $V \times{ }_{X} W$ is a scheme for any pair of maps $V \rightarrow X$ and $W \rightarrow X$ with schemes $V$ and $W$. Such representability was proved in $[\mathrm{K}]$ under quasi-separatedness hypotheses, and is one of the main reasons that quasi-separatedness pervades that work. For the convenience of the reader, we include a proof of this general representability result in A.1, where we also show (without quasi-separatedness hypotheses) that quotients by étale equivalence relations in algebraic spaces are always algebraic spaces.

The avoidance of quasi-separatedness simplifies the discussion of a number of gluing constructions. Beware that if one removes quasi-separatedness from the definition of an algebraic space then some strange things can happen, such as non-quasi-separated algebraic spaces admitting an étale cover by the affine line over a field and unusual behavior for generic points. For this reason, when working with algebraic spaces over a noetherian scheme it is stronger to say "finite presentation" (i.e., finite type and quasi-separated) than "finite type" (even though for schemes there is no distinction over a noetherian base).

Whenever we use a result from $[\mathrm{K}]$ we are either already working with quasi-separated algebraic spaces or it is trivial to reduce the desired assertion to the case of quasi-separated algebraic spaces (such as by 
working étale-locally on the base). Note also that the concept of "algebraic space over a scheme $S$ " in the sense defined in [RG, I, 5.7.1] is the same thing as an algebraic space (as defined above) equipped with a map to $S$.

\section{The EXCELlent CASE}

In this section we prove Theorem 1.2.1 when the algebraic space $S$ is of finite presentation over an excellent noetherian scheme (such as Spec $\mathbf{Z}$ ). This case will be used to deduce the general case in 93 .

We will proceed by noetherian induction on $X$, so first we use deformation theory to show that the result for $X_{\text {red }} \rightarrow S$ implies the result for $X \rightarrow S$. Then we will be in position to begin the induction. The base space $S$ will remain fixed throughout the induction process.

2.1. Reduction to the reduced case. Suppose that Theorem 1.2.1 is proved for $X_{\text {red }} \rightarrow S$ with a fixed noetherian algebraic space $S$. Let us deduce the result for $X \rightarrow S$. We induct on the order of nilpotence of the nilradical of $X$, so we may assume that there is a square-zero coherent ideal sheaf $\mathscr{J}$ on $X$ such that the closed subspace $X_{0} \hookrightarrow X$ defined by killing $\mathscr{J}$ admits an $S$-compactification, say $\sigma: X_{0} \hookrightarrow \bar{X}_{0}$. Let $f_{0}: X_{0} \rightarrow S$ and $f: X \rightarrow S$ be the structure maps.

By blowing up the noetherian $\bar{X}_{0}$ along a closed subspace structure on $\bar{X}_{0}-X_{0}$ (such as the reduced structure) we can arrange that $\bar{X}_{0}-X_{0}$ admits a structure of effective Cartier divisor, so $\sigma$ is an affine morphism. Let us check that it suffices to construct a cartesian diagram of algebraic spaces

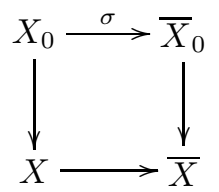

over $S$ in which the bottom arrow is an open immersion and the right vertical arrow is a square-zero closed immersion defined by a quasi-coherent ideal sheaf of $\mathscr{O}_{\bar{X}}$ whose natural $\mathscr{O}_{\bar{X}_{0}}$-module structure is coherent. In such a situation, since the square-zero ideal sheaf $\operatorname{ker}\left(\mathscr{O}_{\bar{X}} \rightarrow \mathscr{O}_{\bar{X}_{0}}\right)$ on $\bar{X}$ is coherent as an $\mathscr{O}_{\bar{X}_{0}}$-module, $\bar{X}$ is necessarily of finite type over $S$ and thus is $S$-proper (since $\bar{X}_{0}$ is $S$-proper). We would therefore be done.

Rather than construct the geometric object $\bar{X}$ directly, we will construct its structure sheaf $\mathscr{O}_{\bar{X}}$ as a square-zero thickening of $\mathscr{O}_{\bar{X}_{0}}$ in a manner that restricts over the open $X_{0} \subseteq \bar{X}_{0}$ to $\mathscr{O}_{X}$ viewed as a squarezero thickening of $\mathscr{O}_{X_{0}}$. The sufficiency of such a sheaf-theoretic approach is provided by an application of Theorem A.4.1 in the noetherian case (also see Remark A.4.2), according to which the existence of diagram (2.1.1) is equivalent to extending the square-zero extension of $f_{0}^{-1}\left(\mathscr{O}_{S}\right)$-algebras

$$
0 \rightarrow \mathscr{J} \rightarrow \mathscr{O}_{X} \rightarrow \mathscr{O}_{X_{0}} \rightarrow 0
$$

on $\left(X_{0}\right)$ ét to a square-zero extension of $\bar{f}_{0}^{-1}\left(\mathscr{O}_{S}\right)$-algebras

$$
0 \rightarrow \overline{\mathscr{J}} \rightarrow \mathscr{A} \rightarrow \mathscr{O}_{\bar{X}_{0}} \rightarrow 0
$$

on $\left(\bar{X}_{0}\right)_{\text {ét }}$ in which the kernel $\overline{\mathscr{J}}$ is coherent as an $\mathscr{O}_{\bar{X}_{0}}$-module.

Since $\sigma$ is affine we have $\mathrm{R}^{1} \sigma_{*, \text { ét }}(\mathscr{J})=0$, so applying $\sigma_{*}$ to (2.1.2) gives a square-zero extension of $\bar{f}_{0}^{-1}\left(\mathscr{O}_{S}\right)$-algebras

$$
0 \rightarrow \sigma_{*}(\mathscr{J}) \rightarrow \sigma_{*}\left(\mathscr{O}_{X}\right) \rightarrow \sigma_{*}\left(\mathscr{O}_{X_{0}}\right) \rightarrow 0
$$

on $\left(\bar{X}_{0}\right)$ ét whose pullback along $\mathscr{O}_{\bar{X}_{0}} \rightarrow \sigma_{*}\left(\mathscr{O}_{X_{0}}\right)$ is a square-zero extension of $\bar{f}_{0}^{-1}\left(\mathscr{O}_{S}\right)$-algebras

$$
0 \rightarrow \sigma_{*}(\mathscr{J}) \rightarrow \mathscr{B} \rightarrow \mathscr{O}_{\bar{X}_{0}} \rightarrow 0
$$

in which the kernel $\sigma_{*}(\mathscr{J})$ is only quasi-coherent as an $\mathscr{O}_{\bar{X}_{0}}$-module. 
By [K, III, Thm. 1.1, Cor. 1.2], we know that $\sigma_{*}(\mathscr{J})=\lim _{\longrightarrow} \overline{\mathscr{J}}_{\alpha}$ where $\overline{\mathscr{J}}_{\alpha}$ ranges through the directed system of $\mathscr{O}_{\bar{X}_{0}}$-coherent subsheaves of $\sigma_{*}(\mathscr{J})$ satisfying $\left.\overline{\mathscr{J}}_{\alpha}\right|_{\left(X_{0}\right)_{\text {ét }}}=\mathscr{J}$. Hence, our problem is reduced to proving bijectivity of the natural map

$$
\stackrel{\lim }{\longrightarrow} \operatorname{Exal}_{\mathscr{O}_{S}}\left(\mathscr{O}_{\bar{X}_{0}}, \mathscr{M}_{i}\right) \rightarrow \operatorname{Exal}_{\mathscr{O}_{S}}\left(\mathscr{O}_{\bar{X}_{0}}, \lim _{\longrightarrow} \mathscr{M}_{i}\right)
$$

for any directed system $\mathscr{M}_{i}$ of quasi-coherent $\mathscr{O}_{\bar{X}_{0}}$-modules. Here, as usual, we let Exal $\mathscr{O}_{S}(\mathscr{A}, \mathscr{M})$ denote the set of isomorphism classes of square-zero extensions of an $\bar{f}_{0}^{-1}\left(\mathscr{O}_{S}\right)$-algebra $\mathscr{A}$ by an $\mathscr{A}$-module $\mathscr{M}$ on $\left(\bar{X}_{0}\right)_{\text {ét }}$ (see for example [EGA, $\left.0_{\mathrm{IV}}, \S 18\right]$ and [I, III.1.1]).

By [I. Thm. III.1.2.3] (applied to the ringed topos $\left(\left(\bar{X}_{0}\right)_{\text {ét }}, \bar{f}_{0}^{-1}\left(\mathscr{O}_{S}\right)\right)$ ), the cotangent complex $\mathrm{L}_{\bar{X}_{0} / S}$ of $\mathscr{O}_{\bar{X}_{0}}$-modules is bounded above and satisfies

$$
\operatorname{Exal}_{\mathscr{O}_{S}}\left(\mathscr{O}_{\bar{X}_{0}}, \mathscr{M}\right) \simeq \operatorname{Ext}_{\mathscr{O}_{\bar{X}_{0}}}^{1}\left(\mathrm{~L}_{\bar{X}_{0} / S}, \mathscr{M}\right)
$$

naturally in any $\mathscr{O}_{\bar{X}_{0}}$-module $\mathscr{M}$. Moreover, by [I, Cor. II.2.3.7] the complex $\mathrm{L}_{\bar{X}_{0} / S}$ has coherent homology modules.

We are now reduced to showing that if $Z$ is any noetherian algebraic space (such as $\bar{X}_{0}$ ) and $\mathscr{F} \bullet$ is any bounded-above complex of $\mathscr{O}_{Z}$-modules with coherent homology modules (such as $\mathrm{L}_{\bar{X}_{0} / S}$ ) then the functor $\operatorname{Ext}_{\mathscr{O}_{Z}}^{j}(\mathscr{F} \bullet, \cdot)$ on quasi-coherent $\mathscr{O}_{Z}$-modules commutes with the formation of direct limits for every $j \in \mathbf{Z}$. This is a standard fact: one reduces first to the case that $\mathscr{F} \bullet=\mathscr{F}[0]$ for a coherent sheaf $\mathscr{F}$ on $Z$, and then uses the the local-to-global Ext spectral sequence and the compatibility of étale cohomology on the qcqs $Z$ with direct limits to reduce to the case of affine $Z$ with the Zariski topology, which is handled by degree-shifting in $j$.

2.2. Reduction to normal case. Now take $S$ to be an algebraic space of finite presentation over an excellent noetherian scheme. In this section we prove the following result.

Proposition 2.2.1. For our fixed $S$, if Theorem 1.2.1 holds whenever $X$ is normal then it holds in general.

Proof. By noetherian induction on $X$ (with its fixed $S$-structure), to prove Theorem 1.2 .1 for $X \rightarrow S$ we may assume that every proper closed subspace of $X$ admits an $S$-compactification. If $X$ is not reduced then 2.1 may be used to conclude the argument, so we may assume that $X$ is reduced.

Let $\pi: \widetilde{X} \rightarrow X$ be the finite surjective normalization, and let $Z \hookrightarrow X$ the closed subspace cut out by the coherent conductor ideal $\operatorname{Ann}_{X}\left(\pi_{*}\left(\mathscr{O}_{\widetilde{X}}\right) / \mathscr{O}_{X}\right)$ of $\widetilde{X}$ over $X$. The open complement $X-Z$ is the maximal open subspace of $X$ over which $\pi$ is an isomorphism. We have $Z \neq X$ since $X$ is reduced (though $Z$ may be non-reduced). By the noetherian induction hypothesis, the separated finite type map $Z \rightarrow S$ admits an $S$-compactification $\bar{Z} \rightarrow S$. Assuming that Theorem 1.2.1 is proved for all normal $S$-separated algebraic spaces of finite type over $S$, so $\widetilde{X}$ admits a compactification $\widetilde{X}^{-}$over $S$, let us see how to construct an $S$-compactification for $X$.

The idea is to reconstruct $X$ from $\widetilde{X}$ via a contraction along the finite surjective map $\pi^{-1}(Z) \rightarrow Z$, and to then apply an analogous such contraction to the $S$-compactification $\widetilde{X}^{-}$of $\widetilde{X}$ (using the $S$-compactification $\bar{Z}$ in place of $Z$ ) to construct an $S$-compactification of $X$. We first record a refinement of a contraction theorem of Artin.

Theorem 2.2.2 (Artin). Let $S$ be an algebraic space of finite presentation over an excellent noetherian scheme, and let

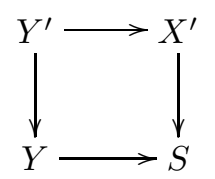

be a commutative diagram of quasi-separated algebraic spaces locally of finite type over $S$, with $Y^{\prime} \rightarrow X^{\prime}$ a closed immersion and $Y^{\prime} \rightarrow Y$ a finite surjective map. 
(1) The pushout $X=Y \coprod_{Y^{\prime}} X^{\prime}$ exists in the category of algebraic spaces, it is quasi-separated and locally of finite type over $S$, and the pushout diagram

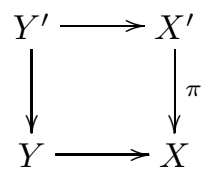

is cartesian, with $Y \rightarrow X$ a closed immersion and $X^{\prime} \rightarrow X$ a finite surjection. If $X^{\prime}$ is $S$-separated (resp. of finite presentation over $S$, resp. $S$-proper) then so is $X$.

(2) The formation of this diagram (as a pushout) commutes with any flat base change on $X$ in the sense that if $X_{1} \rightarrow X$ is a flat map of algebraic spaces then the cartesian diagram

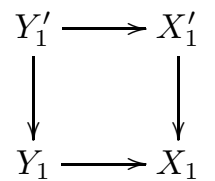

obtained after base change is a pushout diagram in the category of algebraic spaces. In particular, the formation of (2.2.2.1) commutes with étale base change on $X$.

Before we prove Theorem 2.2.2, we make some remarks.

Remark 2.2.3. By descent for morphisms it suffices to prove the result étale-locally on $S$, so the case when $X^{\prime}$ and $Y$ are $S$-separated (which is all we will need in this paper) is easily reduced to the case when $X^{\prime}$ and $Y$ are separated (over $\operatorname{Spec} \mathbf{Z}$ ).

Note also that by taking $X_{1} \rightarrow X$ to be $X-Y \rightarrow X$, it follows that $\pi$ must restrict to an isomorphism over $X-Y$. This will also be evident from how $X$ is constructed.

Proof of Theorem 2.2.2. By working étale-locally on $S$ we may assume that $S$ is an excellent noetherian scheme. Before we address the existence, let us grant existence with $X$ quasi-separated and locally of finite type over $S$ and settle the finer structural properties at the end of part (1) for $X \rightarrow S$. Since $\pi$ will be a finite surjection, the quasi-separated $X$ is necessarily of finite presentation over $S$ (equivalently, is quasicompact) when $X^{\prime}$ is of finite presentation over $S$. Likewise, granting the existence result in general, if $X^{\prime}$ is $S$-separated then the composite of the monomorphism $\Delta_{X / S}: X \rightarrow X \times_{S} X$ with the finite $\pi: X^{\prime} \rightarrow X$ is proper, so $\Delta_{X / S}$ is proper and hence a closed immersion (i.e., $X$ is $S$-separated). Finally, if $X^{\prime}$ is $S$-proper then $X$ is at least $S$-separated, and so $X$ is also $S$-proper since $\pi$ is a finite surjection.

Step 1. We may now turn our attention to the existence problem. As a first step, we establish a pushout property in a special case involving affine schemes. Consider pair of ring maps $B_{1} \rightarrow B_{1}^{\prime}, A_{1}^{\prime} \rightarrow B_{1}^{\prime}$, and form the commutative diagram of affine schemes

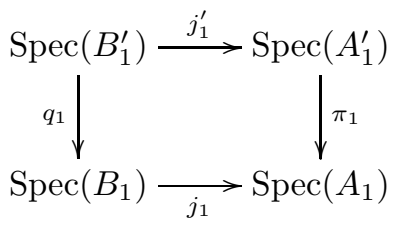

with the fiber product ring $A_{1}:=B_{1} \times_{B_{1}^{\prime}} A_{1}^{\prime}$. Assume that $j_{1}$ is a closed immersion, $\pi_{1}$ is a finite surjection, and that the diagram is cartesian (i.e., the natural map $B_{1} \otimes_{A_{1}} A_{1}^{\prime} \rightarrow B_{1}^{\prime}$ is an isomorphism). For example, these hypotheses hold when $j_{1}^{\prime}$ is a closed immersion and $q_{1}$ is a finite surjection. Indeed, if $B_{1}^{\prime}=A_{1}^{\prime} / J^{\prime}$ then the fiber product ring $A_{1}=B_{1} \times{ }_{A_{1}^{\prime} / J^{\prime}} A_{1}^{\prime}$ satisfies $A_{1} / J=B_{1}$ for the ideal $J=\{0\} \times J^{\prime}$ in $A_{1}$, and since $J A_{1}^{\prime}=J^{\prime}$ we have $B_{1} \otimes_{A_{1}} A_{1}^{\prime}=A_{1}^{\prime} / J A_{1}^{\prime}=A_{1}^{\prime} / J^{\prime}=B_{1}^{\prime}$, so the cartesian property holds. Finally, the natural map $A_{1} \rightarrow A_{1}^{\prime}$ is finite due to finiteness of $B_{1} \rightarrow B_{1}^{\prime}=A_{1}^{\prime} / J^{\prime}$, and in view of the cartesian property the surjectivity of $\pi_{1}$ is reduced to the case of points in Spec $A_{1}$ where $J$ doesn't vanish. At such points $P$ we obtain a local isomorphism since the formation $A_{1}$ is compatible with localization at an element of $A_{1}$ (such as at any $(0, x) \notin P$ with $\left.x \in J^{\prime}\right)$. 
We shall prove that any such diagram 2.2.3.1) is a pushout in the category of algebraic spaces. Fix an algebraic space $T$. We aim to show that the natural map of sets

$$
\rho: \operatorname{Hom}\left(\operatorname{Spec} A_{1}, T\right) \rightarrow \operatorname{Hom}\left(\operatorname{Spec} B_{1}, T\right) \times_{\operatorname{Hom}\left(\operatorname{Spec} B_{1}^{\prime}, T\right)} \operatorname{Hom}\left(\operatorname{Spec} A_{1}^{\prime}, T\right)
$$

is a bijection. Given an algebraic space $Q$ and an $A_{1}$-algebra $C$, write $\underline{Q}(C)$ for the étale sheaf on Spec $A_{1}$ whose value on an étale morphism $W \rightarrow \operatorname{Spec} A_{1}$ is $\operatorname{Hom}\left(W \otimes_{A_{1}} C, Q\right)$. Note that $\underline{Q}\left(A_{1}\right)$ is the restriction of $Q$ to the étale site of $\operatorname{Spec} A_{1}$. We will prove that the map

$$
\underline{\rho}: \underline{T}\left(A_{1}\right) \rightarrow \underline{T}\left(B_{1}\right) \times_{\underline{T}\left(B_{1}^{\prime}\right)} \underline{T}\left(A_{1}^{\prime}\right)
$$

is an isomorphism of sheaves (evaluation on $W=\operatorname{Spec} A_{1}$ recovers $\rho$ ).

Let $(p, q): R \rightarrow U \times U$ be an étale presentation of $T$. Suppose we can show that the natural diagram

$$
\underline{R}\left(B_{1}\right) \times_{\underline{R}\left(B_{1}^{\prime}\right)} \underline{R}\left(A_{1}^{\prime}\right) \Longrightarrow \underline{U}\left(B_{1}\right) \times_{\underline{U}\left(B_{1}^{\prime}\right)} \underline{U}\left(A_{1}^{\prime}\right) \longrightarrow \underline{T}\left(B_{1}\right) \times_{\underline{T}\left(B_{1}^{\prime}\right)} \underline{T}\left(A_{1}^{\prime}\right)
$$

is a colimit diagram in the category of sheaves of sets. There is a map of diagrams

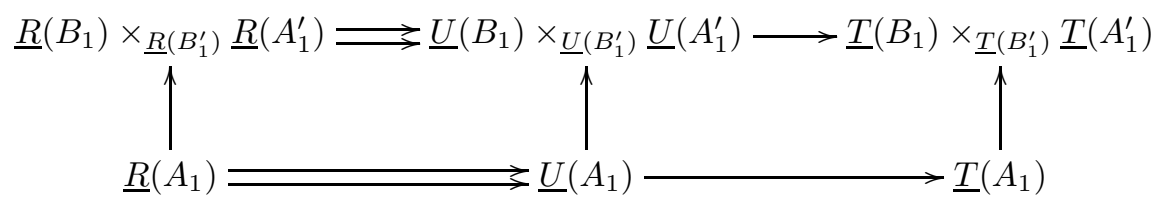

in which we wish to show that the right-most vertical arrow is an isomorphism. If $U$ is a separated scheme (étale cover of $T$ ) then $R$ is a separated scheme. Thus, if we can prove the result when $T$ is a separated scheme, we conclude that the left and middle vertical arrows are isomorphisms, whence the right vertical arrow is an isomorphism because both rows are colimit diagrams of sheaves. But when $T$ is separated and $U$ is affine we have that $R$ is affine. Applying the same argument shows that it suffices to prove the result when $T$ is an affine scheme.

Note that the formation of (2.2.3.1) commutes with affine flat (e.g., affine étale) base change on $A_{1}$ in the following sense. Let $\operatorname{Spec} A_{2} \rightarrow \operatorname{Spec} A_{1}$ be flat and define $A_{2}^{\prime}=A_{2} \otimes_{A_{1}} A_{1}^{\prime}$ and similarly for $B_{2}^{\prime}$ and $B_{2}$. The natural exact sequence of $A_{1}$-modules

$$
0 \rightarrow B_{1} \times{ }_{B_{1}^{\prime}} A_{1}^{\prime} \rightarrow B_{1} \times A_{1}^{\prime} \rightarrow B_{1}^{\prime}
$$

remains exact after scalar extension by $A_{1} \rightarrow A_{2}$, so the natural map $A_{2} \rightarrow B_{2} \times_{B_{2}^{\prime}} A_{2}^{\prime}$ is an isomorphism. Since the morphism $\underline{\rho}$ is an isomorphism if and only if it induces bijections when evaluated on all étale $A_{1}$-algebras $A_{1} \rightarrow A_{2}$, we are done (when $T$ is affine) because we know (2.2.3.1) is a pushout in the category of affine schemes.

Thus, it suffices to show that (2.2.3.2) is a colimit diagram (of sheaves). Suppose $\left(t_{1}, t_{2}\right)$ is a section of $\underline{T}\left(B_{1}\right) \times_{\underline{T}\left(B_{1}^{\prime}\right) \underline{T}}\left(A_{1}^{\prime}\right)$ over an étale $A_{1}$-scheme $W$. Since all of the schemes in diagram (2.2.3.1) are finite over Spec $A_{1}$, we know that their pullbacks along any strict henselization of $A_{1}$ split as finite products of strictly henselian local rings EGA, $\left.\mathrm{IV}_{4}, 18.8 .10\right]$. Thus, since $U \rightarrow T$ is an étale surjection (hence locally of finite presentation), after replacing $W$ by an étale cover we can choose a lift $\tilde{t}_{1} \in \underline{U}\left(B_{1}\right)(W)$ of $t_{1}$. We now have a commutative diagram

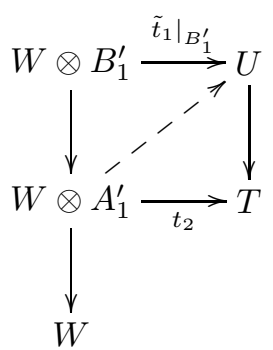

in which the top left vertical arrow is a closed immersion, the bottom left vertical arrow is a finite surjection, and the right vertical arrow is an étale surjection. If $W \otimes A_{1}^{\prime}$ were strictly henselian, a unique dotted arrow 
in the commutative diagram would exist. Thus, for any strictly henselian local $W$-scheme $H \rightarrow W$, the restricted diagram

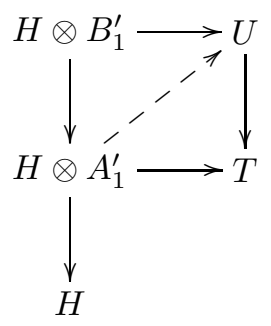

admits a unique dotted lift. Since $U \rightarrow T$ is locally of finite presentation, it follows that there is an étale surjection $W^{\prime} \rightarrow W$ such that there is a dotted lift in the resulting diagram

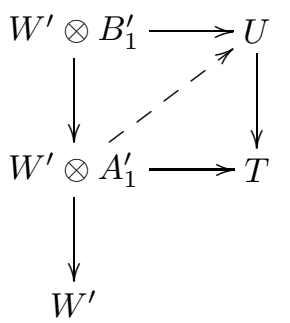

completing the proof that the right arrow of diagram (2.2.3.2) is a surjection of étale sheaves.

Now suppose $\left(u_{1}, v_{1}\right)$ and $\left(u_{2}, v_{2}\right)$ are two sections of $\underline{U}\left(B_{1}\right) \times_{\underline{U}\left(B_{1}^{\prime}\right)} \underline{U}\left(A_{1}^{\prime}\right)$ over $W \rightarrow$ Spec $A_{1}$ with the same image in $\underline{T}\left(B_{1}\right) \times_{\underline{T}\left(B_{1}^{\prime}\right)} \underline{T}\left(A_{1}^{\prime}\right)$. Since $R \rightarrow U \times_{T} U$ is an isomorphism, we know that there are unique sections $r_{1} \in \underline{R}\left(B_{1}\right)(W)$ and $r_{2} \in \underline{R}\left(A_{1}^{\prime}\right)(W)$ such that $p\left(r_{i}\right)=u_{i}$ and $q\left(r_{i}\right)=v_{i}$. Moreover, these sections restrict to sections $r \in \underline{R}\left(B_{1}^{\prime}\right)(W)$ such that $p(r)=\left.u_{1}\right|_{B_{1}^{\prime}}=\left.u_{2}\right|_{B_{1}^{\prime}}$ and $q(r)=\left.v_{1}\right|_{B_{1}^{\prime}}=\left.v_{2}\right|_{B_{1}^{\prime}}$. Since any such $r$ is unique, we see that $\left(r_{1}, r_{2}\right)$ defines the unique section of $\underline{R}\left(B_{1}\right) \times_{\underline{R}}\left(B_{1}^{\prime}\right) \underline{R}\left(A_{1}^{\prime}\right)$ over $W$ carrying $\left(u_{1}, v_{1}\right)$ to $\left(u_{2}, v_{2}\right)$. This completes the proof that (2.2.3.2) is a colimit diagram and thus the proof that (2.2.3.1) is a pushout diagram in the category of algebraic spaces.

Step 2. The main work is to handle the case when $X^{\prime}$ (and hence $Y^{\prime}$ and $Y$ ) is of finite presentation over $S$, with $X$ constructed as also finitely presented over $S$; the existence result more generally will then be obtained by simple gluing arguments. Thus, we now assume (until said otherwise) that $X^{\prime}, Y^{\prime}$, and $Y$ are of finite presentation over $S$. In this situation, the existence of the pushout in the category of quasi-separated algebraic spaces is [A Thm. 6.1] when $S$ is of finite presentation over a field or excellent Dedekind domain, and the construction in its proof gives that (i) $X$ is of finite presentation over $S$, (ii) the diagram (2.2.2.1) is cartesian, (iii) $Y \rightarrow X$ is a closed immersion, and (iv) $\pi: X^{\prime} \rightarrow X$ is proper and restricts to an isomorphism over the open subspace $X-Y$. Since $\pi$ is clearly quasi-finite (by the cartesian property), it must also be finite.

Artin assumed $S$ is of finite presentation over a field or excellent Dedekind domain (rather than over an arbitrary excellent noetherian scheme) because his criterion for a functor to be an algebraic space was originally proved only for such $S$. By [CdJ, Thm. 1.5] Artin's proof of that criterion works for any excellent noetherian $S$, so likewise the above conclusions hold in such generality. The pushout constructed in the finitely presented case has only been shown to be a pushout in the category of quasi-separated algebraic spaces, as this is the situation considered by Artin. To establish the pushout property relative to maps to any algebraic space, the key step is to check, as we shall now do (assuming $X^{\prime}, Y^{\prime}$, and $Y$ are finitely presented over $S$ ), that the pushout property of $X$ is preserved by any quasi-separated étale localization on $X$. Artin's construction of the quasi-separated pushout $X$ is via an indirect algebraization process, so to be rigorous will require some care. If $X_{1} \rightarrow X$ is a quasi-separated étale map, to prove that the $X_{1}$-pullback diagram is a pushout it is enough (by étale descent for morphisms) to check this property étale-locally on $X_{1}$. More specifically, it suffices to treat the case when $X_{1}=\operatorname{Spec} A_{1}$ is affine. 
Consider the resulting pullback diagram (2.2.2.2) which consists of affine schemes, say with $Y_{1}=\operatorname{Spec} B_{1}$, $X_{1}^{\prime}=\operatorname{Spec} A_{1}^{\prime}$, and $Y_{1}^{\prime}=\operatorname{Spec} B_{1}^{\prime}$. We claim that the natural map $\theta_{1}: A_{1} \rightarrow B_{1} \times_{B_{1}^{\prime}} A_{1}^{\prime}$ is an isomorphism. Let $J_{1}=\operatorname{ker}\left(A_{1} \rightarrow B_{1}\right)$, so $B_{1}^{\prime}=A_{1}^{\prime} / J_{1} A_{1}^{\prime}$. Since $A_{1}^{\prime}$ is $A_{1}$-finite, $\theta_{1}$ is at least finite. Also, $\operatorname{Spec}\left(\theta_{1}\right)$ is clearly an isomorphism over the open complement of $\operatorname{Spec} B_{1}=\operatorname{Spec}\left(A_{1} / J_{1}\right)$ in $\operatorname{Spec} A_{1}$. Hence, to prove that $\theta_{1}$ is an isomorphism it suffices to show that the induced map $\widehat{\theta}_{1}$ between $J_{1}$-adic completions is an isomorphism.

Write $\widehat{A}_{1}$ and $\widehat{A}_{1}^{\prime}$ to denote the $J_{1}$-adic and $J_{1} A_{1}^{\prime}$-adic completions respectively, and let the formal algebraic space $\mathfrak{X}^{\prime}$ denote the formal completion of $X^{\prime}$ along $Y^{\prime}$. The étale map Spec $B_{1} \rightarrow Y$ has pullback along $Y^{\prime} \rightarrow Y$ identified with Spec $B_{1}^{\prime} \rightarrow Y^{\prime}$, and (using Proposition A.1.3) the unique lifting of this latter étale map to a formal noetherian algebraic space formally étale over $\mathfrak{X}^{\prime}$ is uniquely identified with $\operatorname{Spf}\left(\widehat{A}_{1}^{\prime}\right) \rightarrow \mathfrak{X}^{\prime}$. Artin's construction of $X$ identifies $\widehat{A}_{1}$ with the ring-theoretic fiber product over $B_{1}^{\prime}$ of $B_{1}$ against the coordinate ring $\widehat{A}_{1}^{\prime}$ of this formal lifting, which is to say that the natural map $\widehat{A}_{1} \rightarrow B_{1} \times_{B_{1}^{\prime}} \widehat{A}_{1}^{\prime}$ is an isomorphism. This isomorphism is the map $\widehat{\theta}_{1}$, so $\widehat{\theta}_{1}$ is an isomorphism and hence $\theta_{1}$ is an isomorphism.

With $\theta_{1}$ now shown to be an isomorphism, the verification of the quasi-separated pushout property after base change to $X_{1}$ is a special case of a general pushout property for ring-theoretic fiber products that we settled in Step 1 (since Artin's pushout diagram is also cartesian). Since any quasi-separated étale base change on $X$ has now been shown to yield a pushout diagram in the category of quasi-separated algebraic spaces, to prove that Artin's quasi-separated pushout is actually a pushout in the category of all algebraic spaces we can use étale descent for morphisms to formally reduce to the special case when $X^{\prime}, Y$, and $Y^{\prime}$ are affine. In this affine case we can form the diagram as in (2.2.3.1) except with $q_{1}$ a finite surjection and $j_{1}^{\prime}$ a closed immersion (and $A_{1}:=B_{1} \times_{B_{1}^{\prime}} A_{1}^{\prime}$ ). As we saw early in Step 1, in such cases necessarily $j_{1}$ is a closed immersion, $\pi_{1}$ is a finite surjection, and the diagram is cartesian. Hence, by Step 1 the affine scheme $\operatorname{Spec}\left(A_{1}\right)$ is a pushout in the category of all algebraic spaces. This affine scheme is quasi-separated, so it must coincide with the pushout already constructed in the category of quasi-separated algebraic spaces. Hence, this latter pushout is also a pushout in the category of all algebraic spaces. The proof of compatibility with quasi-separated étale base now applies verbatim to arbitrary étale base change.

Step 3. We continue to assume that $X^{\prime}, Y^{\prime}$, and $Y$ are finitely presented over $S$, and now we improve upon Step 2 by showing that the formation of $X$ is compatible with any flat base change $X_{1} \rightarrow X$ (in the sense that the $X_{1}$-pullback diagram is a pushout in the category of algebraic spaces). By étale descent for morphisms, coupled with the established compatibility with étale base change on $X$, we are reduced to the case when $X$ is an affine scheme (so $Y, Y^{\prime}$, and $X^{\prime}$ are also affine) and $X_{1}$ is affine. Say $X=\operatorname{Spec} A$, $Y=\operatorname{Spec} B, X^{\prime}=\operatorname{Spec} A^{\prime}, Y^{\prime}=\operatorname{Spec} B^{\prime}$, and $X_{1}=\operatorname{Spec} A_{1}$. In the commutative diagram of noetherian rings

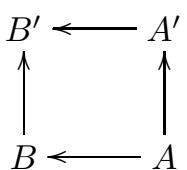

the vertical maps are finite, the horizontal maps are surjective, $B^{\prime}=A^{\prime} \otimes_{A} B$, and (as in Step 2) the natural map of rings $\phi: A \rightarrow B \times{ }_{B^{\prime}} A^{\prime}$ is an isomorphism. Let $A_{1}^{\prime}:=A_{1} \otimes_{A} A^{\prime}$, and similarly for $B_{1}$ and $B_{1}^{\prime}$. By the same calculation with flat scalar extension as in Step 1, the natural map $A_{1} \rightarrow B_{1} \times_{B_{1}^{\prime}} A_{1}^{\prime}$ is an isomorphism. Thus, in view of the general pushout result proved in Step 1, we have established compatibility with any flat base change on $X$ (when $X^{\prime}, Y^{\prime}$, and $Y$ are finitely presented over $S$ ).

Theorem 2.2.2 has now been proved when $X^{\prime}, Y^{\prime}$, and $Y$ are finitely presented over $S$. It remains to handle the existence and compatibility with flat base change of a quasi-separated pushout $X$ when the quasi-separated $X^{\prime}, Y^{\prime}$, and $Y$ are merely locally of finite type over $S$. (Note that finite presentation is the same as finite type for algebraic spaces quasi-separated over $S$.)

The proof of existence will proceed by a gluing method, and the compatibility with flat base change on $X$ in general will follow immediately from the gluing construction of $X$ and such compatibility in the finitely presented case. The key point is that the construction given above in the finitely presented case is well-behaved with respect to Zariski localization on the source. More precisely, we have: 
Lemma 2.2.4. In the setup of Theorem 2.2.2, let $V^{\prime} \subseteq X^{\prime}$ and $W \subseteq Y$ be open subspaces such that $\pi^{-1}(W)=Y^{\prime} \cap V^{\prime}$ as open subspaces of $Y^{\prime}$. Then the natural map of pushouts

$$
W \coprod_{\pi^{-1}(W)} V^{\prime} \rightarrow Y \coprod_{Y^{\prime}} X^{\prime}
$$

is an open immersion.

Since we have only proved Theorem 2.2 .2 in the finitely presented case (over $S$ ), the lemma can only be proved at this point in such cases (i.e., $X^{\prime}, Y^{\prime}, Y, V^{\prime}$, and $W$ are finitely presented over $S$ ). However, the proof goes the same way in this case as it will in the general case, so we write one argument below that is applied first in the finitely presented case and then (after general existence as in Theorem 2.2.2(1) is proved) in the general case.

Proof. By Theorem 2.2.2(2) the formation of the pushout is compatible with flat base change on $Y \coprod_{Y^{\prime}} X^{\prime}$, so by working étale-locally on this pushout we may reduce to the case when it is affine. In other words, we have $X^{\prime}=\operatorname{Spec}\left(A^{\prime}\right), Y^{\prime}=\operatorname{Spec}\left(B^{\prime}\right), Y=\operatorname{Spec}(B)$, and $Y \coprod_{Y^{\prime}} X^{\prime}=\operatorname{Spec}(A)$ with $A=B \times_{B^{\prime}} A^{\prime}, B \rightarrow B^{\prime}$ finite, $A^{\prime} \rightarrow B^{\prime}$ surjective, and $B^{\prime}=B \otimes_{A} A^{\prime}$ (so $A \rightarrow A^{\prime}$ is finite and $A \rightarrow B$ is surjective). In particular, $X^{\prime} \rightarrow X$ is an isomorphism over $X-Y$.

The condition $\pi^{-1}(W)=Y^{\prime} \cap V^{\prime}$ implies that $V^{\prime}=\pi^{-1}\left(\pi\left(V^{\prime}\right)\right)$, so since $\pi$ is a surjective finite map we see that $V:=\pi\left(V^{\prime}\right)$ is an open subset of $X=\operatorname{Spec}(A)$ with complement $Y-W$. Giving $V$ the open subscheme structure, we want the commutative diagram

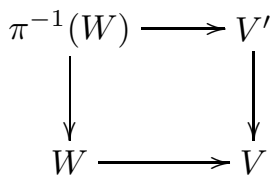

to be a pushout. That is, we want the natural map from the algebraic space $P:=W \coprod_{\pi^{-1}(W)} V^{\prime}$ to the scheme $V$ to be an isomorphism. We may work Zariski-locally on $V$ due to the flat base change compatibility of pushouts, so we may assume $V=\operatorname{Spec}\left(A_{a}\right)$ for some $a \in A=B \times{ }_{B^{\prime}} A^{\prime}$. Writing $a=\left(b, a^{\prime}\right)$ where $b$ and $a^{\prime}$ have the same image $b^{\prime}$ in $B^{\prime}$, clearly $A_{a}=B_{b} \times_{B_{b^{\prime}}^{\prime}} A_{a^{\prime}}^{\prime}$. But $\operatorname{Spec}\left(A_{a^{\prime}}^{\prime}\right)$ is the preimage $V^{\prime}$ of $V$ in $X^{\prime}$ and $\operatorname{Spec}\left(B_{b}\right)$ is the preimage $W$ of $V$ in $Y$, so the isomorphism property for $P \rightarrow V$ is reduced to the affine cases for which the pushout has already been shown to be given by a ring-theoretic fiber product.

To complete the proof of existence of $Y \coprod_{Y^{\prime}} X^{\prime}$ as a quasi-separated algebraic space locally of finite type over $S$ in general, let $\left\{U_{i}\right\}$ be a Zariski-open covering of $Y$ by quasi-compact opens, and let $\left\{U_{i}^{\prime}\right\}$ be the pullback cover of $Y^{\prime}$. Each $U_{i}^{\prime}$ has the form $U_{i}^{\prime}=Y^{\prime} \cap V_{i}^{\prime}$ for a quasi-compact open subspace $V_{i}^{\prime} \subseteq X^{\prime}$. Thus, we can form pushouts $V_{i}:=U_{i} \coprod_{U_{i}^{\prime}} V_{i}^{\prime}$ of finite presentation over $S$. Define $U_{i j}=U_{i} \cap U_{j}, U_{i j}^{\prime}=U_{i}^{\prime} \cap U_{j}^{\prime}$, and $V_{i j}^{\prime}=V_{i}^{\prime} \cap V_{j}^{\prime}$. We may form the pushout $V_{i j}:=U_{i j} \coprod_{U_{i j}^{\prime}} V_{i j}^{\prime}$ and by Lemma 2.2.4 the natural maps $V_{i j} \rightarrow V_{i}$ and $V_{i j} \rightarrow V_{j}$ are quasi-compact open immersions. It is trivial to check the triple overlap compatibility, and so we may glue the $V_{i}$ 's to obtain a quasi-separated algebraic space $V$ locally of finite type over $S$ equipped with a closed immersion $U \hookrightarrow V$ and a finite surjection $V^{\prime} \rightarrow V$ with respect to which $V$ satisfies the universal property of $Y \coprod_{Y^{\prime}} V^{\prime}$ where $V^{\prime}=\cup V_{i}^{\prime}$. Either by construction or flat base change compatibility (relative to $V-Y \rightarrow V$ ), the finite surjection $V^{\prime} \rightarrow V$ restricts to an isomorphism over $V-Y$. Hence, we may glue $V$ and $X^{\prime}$ along the common open subspace $V-Y \simeq \pi^{-1}(V-Y)=V^{\prime}-Y^{\prime} \subset X^{\prime}-Y^{\prime}$ inside of $V$ and $X^{\prime}$. This gluing is the required $X$ and satisfies all of the desired properties.

As an application of Theorem 2.2.2, we can now give a pushout method to reconstruct certain reduced algebraic spaces from their normalization and their non-normal locus.

Corollary 2.2.5. Let $X$ be a reduced quasi-separated algebraic space locally of finite type over an excellent scheme, and let $\pi: \widetilde{X} \rightarrow X$ denote the normalization. Let $j: Z \hookrightarrow X$ be the closed subspace cut out by the conductor of $\widetilde{X} / X$. Let $Y=Z \times_{X} \widetilde{X}$. The natural map $Z \coprod_{Y} \widetilde{X} \rightarrow X$ is an isomorphism. 
Proof. By Theorem 2.2.2 the formation of the pushout $Z \coprod_{Y} \widetilde{X}$ commutes with étale localization on the pushout, and in particular with étale localization on $X$. Since the formation of the conductor is étale-local on $X$, it suffices to treat the case when $X=\operatorname{Spec} A$ is affine, so $\widetilde{X}=\operatorname{Spec} \widetilde{A}$ for the normalization $\widetilde{A}$ of $A$, and $Z=\operatorname{Spec}(A / J)$ and $Y=\operatorname{Spec}(\widetilde{A} / J)$ for the conductor ideal $J \subset A$ (so that $J$ is also an ideal of $\widetilde{A}$ ). The argument in Step 1 of the proof of Theorem 2.2.2 shows that in this case the pushout is identified with $\operatorname{Spec}((A / J) \times \widetilde{A} / J) \widetilde{A})$, so our problem is to prove that the natural map

$$
h: A \rightarrow C:=(A / J) \times \widetilde{A} / J
$$

is an isomorphism.

Since $\widetilde{A}$ is $A$-finite, it is obvious that $h$ is finite. Also, $h$ is injective since $A \rightarrow \widetilde{A}$ is injective (as $A$ is reduced). On the other hand, since $J$ is an ideal of both $A$ and $\widetilde{A}$, it is a trivial calculation (using the explicit description of the fiber product of rings) that $h$ is surjective.

Now we return to the setup with noetherian induction preceding the statement of Theorem 2.2.2, Just as Corollary 2.2.5 reconstructs $X$ from $\widetilde{X}$ by contracting along the canonical finite surjective map $\pi: Y=$ $Z \times_{X} \widetilde{X} \rightarrow Z$, we aim to construct an $S$-compactification of $X$ by contracting a suitable choice of $\widetilde{X}^{-}$along a finite surjective map $\bar{\pi}: \bar{Y} \rightarrow \bar{Z}$, where $\bar{Y}$ is the closure of $Y$ in $\widetilde{X}^{-}$. The first step is to construct $\bar{\pi}$ extending $\left.\pi\right|_{Y}$.

Lemma 2.2.6. For a suitable choice of schematically dense open immersions $\widetilde{X} \hookrightarrow \widetilde{X}^{-}$and $Z \hookrightarrow \bar{Z}$ over $S$ into $S$-proper algebraic spaces, the schematic closure $\bar{Y}$ of $Y:=Z \times_{X} \widetilde{X}$ in $\widetilde{X}^{-}$admits a finite surjective $S$-map $\bar{\pi}: \bar{Y} \rightarrow \bar{Z}$ which restricts to $\pi: Y \rightarrow Z$ over the open subspace $Z \subseteq \bar{Z}$.

Proof. We make an initial choice of $S$-compactifications $\widetilde{X} \hookrightarrow \widetilde{X}_{1}^{-}$and $Z \hookrightarrow \bar{Z}_{1}$, which we may and do arrange to be schematically dense, and we define $\bar{Y}_{1}$ to be the schematic closure of $Y$ in $\widetilde{X}_{1}^{-}$. Let $\bar{Y}^{\prime}$ denote the $S$-proper schematic closure of $Y$ in $\bar{Y}_{1} \times_{S} \bar{Z}_{1}$. The natural map $q^{\prime}: \bar{Y}^{\prime} \rightarrow \bar{Y}_{1}$ restricts to an isomorphism over the open subspace $Y \subseteq \bar{Y}_{1}$ because $Y \rightarrow Y \times_{S} \bar{Z}_{1}$ is a closed immersion (as it is the graph of an $S$-map $Y \rightarrow Z \hookrightarrow \bar{Z}_{1}$ to an $S$-separated target). Likewise, due to the definition of $\bar{Y}^{\prime}$ as a scheme-theoretic closure, the natural proper $S$-map $\pi^{\prime}: \bar{Y}^{\prime} \rightarrow \bar{Z}_{1}$ restricts to $\pi$ over the open subspace $Z \subseteq \bar{Z}_{1}$ because the monomorphism $Y \rightarrow \bar{Y}^{\prime} \times_{S} Z$ is a closed immersion (as it is finite, due to finiteness of $\pi: Y \rightarrow Z$ ).

Now we use some results of Raynaud and Gruson concerning the use of blow-ups of algebraic spaces to "improve" properties of morphisms. Since the proper map $q^{\prime}: \bar{Y}^{\prime} \rightarrow \bar{Y}_{1}$ restricts to an isomorphism over the open subspace $Y \subseteq \bar{Y}_{1}$, by [RG, I, 5.7.12] there is a blow-up $q^{\prime \prime}: \bar{Y}^{\prime \prime} \rightarrow \bar{Y}^{\prime}$ with center disjoint from $Y$ such that $q^{\prime} \circ q^{\prime \prime}: \bar{Y}^{\prime \prime} \rightarrow \bar{Y}_{1}$ is a blow-up with center disjoint from $Y$. (Blow-ups away from $Y$ are easier to work with than general morphisms that are isomorphisms over $Y$, since we can focus attention on the center of the blow-up.)

Let $\pi^{\prime \prime}=\pi^{\prime} \circ q^{\prime \prime}: \bar{Y}^{\prime \prime} \rightarrow \bar{Z}_{1}$ denote the natural composite map, so this restricts to the finite map $\pi$ over $Z \subseteq \bar{Z}_{1}$. Hence, by [RG, I, 5.7.10], there is a blow-up $g: \bar{Z} \rightarrow \bar{Z}_{1}$ with center disjoint from $Z$ such that the strict transform $\varphi: \bar{Y} \rightarrow \bar{Y}^{\prime \prime}$ of $\pi^{\prime \prime}$ with respect to $g$ has $\bar{Y}$ finite over $\bar{Z}$. (Note that $\varphi$ is a blow-up of $\bar{Y}^{\prime \prime}$ with center disjoint from $Y$; see [C2, Lemma 1.1] for a proof which adapts immediately to the case of algebraic spaces. Also, since $Z$ is a schematically dense open in $\bar{Z}_{1}$, it is a schematically dense open in $\bar{Z}$.) By construction, the finite map $\bar{\pi}: \bar{Y} \rightarrow \bar{Z}$ restricts to $\pi$ over $Z$, and $Y$ and $Z$ are respectively schematically dense open subspaces in the $S$-proper $\bar{Y}$ and $\bar{Z}$. Since $\pi$ is surjective, it follows from the schematic density of $Z$ in $\bar{Z}$ that $\bar{\pi}$ is surjective.

The composite map $\left(q^{\prime} \circ q^{\prime \prime}\right) \circ \varphi: \bar{Y} \rightarrow \bar{Y}_{1}$ is a composite of blow-ups with center disjoint from $Y$, so by [RG, I, 5.1.4] (cf. [C2, Lemma 1.2] for a more detailed proof, which carries over to the case of algebraic spaces with the help of [RG, I, 5.7.8]) it is itself a blow-up along a closed subspace $C \subseteq \bar{Y}_{1}$ disjoint from $Y$. Since $\widetilde{X} \cap \bar{Y}_{1}=Y$ as open subspaces of $\bar{Y}_{1}$, when $C$ is viewed as a closed subspace of $\widetilde{X}_{1}^{-}$it is disjoint from the open subspace $\widetilde{X}$. Thus, since $\widetilde{X}$ is a schematically dense open in $\widetilde{X}_{1}^{-}$, the blow-up $\widetilde{X}^{-}:=\mathrm{Bl}_{C}\left(\widetilde{X}_{1}^{-}\right)$is an $S$-proper algebraic space naturally containing $\widetilde{X}$ as a schematically dense open subspace over $S$. Exactly 
as in the case of schemes (see C2, Lemma 1.1]), the blow-up $\bar{Y}=\operatorname{Bl}_{C}\left(\bar{Y}_{1}\right)$ is naturally a closed subspace of the blow-up $\widetilde{X}^{-}$. Hence, $\bar{Y}$ must be the schematic closure of $Y$ in $\widetilde{X}^{-}$(since $Y$ is a schematically dense open in $\bar{Y}_{1}$ ). Since $\bar{Y}$ was constructed to admit the desired $\bar{\pi}$, we are done.

Using $S$-compactifications as in Lemma 2.2.6, define the pushout algebraic space

$$
\bar{X}:=\bar{Z} \coprod_{\bar{Y}} \tilde{X}^{-} \text {. }
$$

This is a pushout of the sort considered in Theorem 2.2.2, By Theorem 2.2.2, $\bar{X}$ is $S$-proper. By Corollary 2.2 .5 and the functoriality of pushouts, there is a natural $S$-map

$$
j: X \simeq Z \coprod_{Y} \tilde{X} \rightarrow \bar{Z} \coprod_{\bar{Y}} \tilde{X}^{-}=: \bar{X} .
$$

Thus, to complete the reduction of the proof of Theorem 1.2.1 over $S$ to the case when $X$ is normal, it suffices to prove that $j$ is an open immersion. Since $\bar{\pi}^{-1}(Z)=Y=\bar{Y} \cap X$ as open subspaces of $\bar{Y}$, this is a special case of Lemma 2.2.4.

This completes the proof of Proposition 2.2.1.

To prove the general case of Theorem 1.2.1 we need a few results on quotients.

2.3. Group quotients. The proof of Theorem 1.2.1 for normal $X$ (and $S$ a noetherian scheme) in R1] rests on a group quotient result that we shall find useful for other purposes, so we now wish to record it. Rather generally, if $X^{\prime}$ is a quasi-separated algebraic space equipped with an action by a finite group $G$, we define the quotient $X^{\prime} / G$ (if it exists) to be an initial object $X^{\prime} \rightarrow X^{\prime} / G$ in the category of quasiseparated algebraic spaces equipped with a $G$-invariant map from $X^{\prime}$ provided that (in addition) the map of sets $X^{\prime}(k) / G \rightarrow\left(X^{\prime} / G\right)(k)$ is bijective for all algebraically closed fields $k$. (It makes sense to drop the quasi-separated hypotheses, but we only know an existence result for such quotients using a universal mapping property within the category of quasi-separated algebraic spaces.) Note that if $X^{\prime}$ is reduced and $X^{\prime} / G$ exists then $X^{\prime} / G$ must be reduced since $X^{\prime} \rightarrow\left(X^{\prime} / G\right)_{\text {red }}$ is easily shown to satisfy the same universal property. Such quotients are useful for relating construction problems for normal noetherian algebraic spaces to analogous problems for normal noetherian schemes, due to the following result.

Proposition 2.3.1. Let $X$ be a (non-empty) connected normal noetherian algebraic space. There exists a connected normal noetherian scheme $X^{\prime}$ equipped with a right action by a finite group $G$ and a finite $G$ invariant map $\pi: X^{\prime} \rightarrow X$ such that $\pi$ is finite étale $G$-torsor over a dense open subspace of $X$ and exhibits $X$ as $X^{\prime} / G$. (In particular, $X^{\prime} / G$ exists.)

This result is [LMB, Cor. 16.6.2], and it is also proved in [R1, Cor.]; in both of these references, the existence of $X^{\prime} / G$ is part of the assertion. For the convenience of the reader, we give a proof after we first discuss the general existence problem for $X^{\prime} / G$ when one does not have a candidate for this quotient already in hand. Such an existence result is required for applications to compactification. Deligne proved the existence of $X^{\prime} / G$ when $X^{\prime}$ is a separated algebraic space; this is recorded in Theorem 3.1 .13 below and is not used in this paper. We wish to avoid such separatedness hypotheses on total spaces, only ever assuming separatedness for morphisms (if at all).

The best approach we know for existence results for quotients $X^{\prime} / G$ is to use the work of Keel and Mori [KM] (or its generalizations; see Ry1]) on coarse moduli spaces for Artin stacks. This allows one to relate the quotient $X^{\prime} / G$ to the Deligne-Mumford stack $\left[X^{\prime} / G\right]$. It is therefore convenient to now recall the definition and main existence theorem for coarse moduli spaces of quasi-separated Artin stacks.

If $\mathscr{X}$ is a quasi-separated Artin stack then a coarse moduli space is a morphism $\pi: \mathscr{X} \rightarrow X$ to a quasiseparated algebraic space $X$ such that it is initial in the category of maps from $\mathscr{X}$ to quasi-separated algebraic spaces and the map of sets $(\mathscr{X}(k) / \simeq) \rightarrow X(k)$ is bijective for every algebraically closed field $k$. It was proved by Keel and Mori [KM] that there exists a coarse moduli space $X$ whenever $\mathscr{X}$ is of finite presentation (and hence quasi-separated) over a locally noetherian scheme $S$ and the inertia stack $I_{S}(\mathscr{X})=\mathscr{X} \times \mathscr{X} \times \mathscr{X}_{\mathscr{X}}$ is $\mathscr{X}$-finite (under either projection map). Moreover, it is proved there that the following additional properties 
hold in such cases: $\pi$ is proper and quasi-finite, $X$ is of finite presentation over $S, X$ is $S$-separated if $\mathscr{X}$ is $S$-separated, and the formation of $\pi$ commutes with any quasi-separated flat base change morphism $X^{\prime} \rightarrow X$ that is locally of finite type (i.e., $X^{\prime}$ is the coarse moduli space of the quasi-separated Artin stack $\mathscr{X} \times{ }_{X} X^{\prime}$ ). These results were generalized in [C3] and Ry1, where the noetherian hypotheses were eliminated, but the above results for $\mathscr{X}$ finitely presented over a locally noetherian scheme are enough for what we need. Note that a special case of the compatibility with quasi-separated flat base change locally of finite type is that the formation of the coarse moduli space $X$ is compatible with quasi-separated étale base change on $X$.

By using the universal properties of coarse moduli spaces and quotient stacks, one easily proves:

Lemma 2.3.2. Let $Y^{\prime}$ be a quasi-separated algebraic space equipped with an action by a finite group $H$. The quotient $Y^{\prime} / H$ exists if and only if the Deligne-Mumford stack $\left[Y^{\prime} / H\right]$ admits a coarse moduli space $Q$, in which case the natural map $Y^{\prime} / H \rightarrow Q$ is an isomorphism.

We shall be interested in the special case of Lemma 2.3.2 when $Y^{\prime}$ is separated of finite presentation (even finite) over a noetherian algebraic space $S$ and $H$ acts on $Y^{\prime}$ over $S$. In this case the quotient stack $\left[Y^{\prime} / H\right]$ is of finite presentation over $S$ with diagonal $\Delta_{\left[Y^{\prime} / H\right] / S}$ that is separated (as it is a subfunctor of the separated Isom-functor between pairs of $H$-torsors over $S$-schemes), and the projections $I_{S}\left(\left[Y^{\prime} / H\right]\right) \rightrightarrows\left[Y^{\prime} / H\right]$ are finite (using the fact that $Y^{\prime}$ is separated). Hence, if $S$ is a scheme then by $\left[\mathrm{KM}\right.$ the quotient $Y^{\prime} / H$ does exist as an algebraic space of finite presentation over $S$, and the map $Y^{\prime} \rightarrow Y^{\prime} / H$ is a finite surjection because $Y^{\prime} \rightarrow\left[Y^{\prime} / H\right]$ is an $H$-torsor and $\left[Y^{\prime} / H\right] \rightarrow Y^{\prime} / H$ is a proper quasi-finite surjection.

In particular, since $Y^{\prime}$ is $S$-separated the quotient $Y^{\prime} / H$ is also $S$-separated (as could also be deduced from $S$-separatedness of $\left[Y^{\prime} / H\right]$ ), so if $Y^{\prime}$ is $S$-proper then $Y^{\prime} / H$ is also $S$-proper. The same conclusions hold if $S$ is merely a noetherian algebraic space rather than a noetherian scheme. Indeed, since quotients by étale equivalence relations always exist in the category of algebraic spaces (Corollary A.1.2), the étale-localization compatibility of the formation of coarse spaces in the setup of [KM] allows us to work étale-locally over $S$ (and to thereby reduce to the case when $S$ is a noetherian scheme) for the existence result as well as for the finer asserted properties of the quotient over $S$. The following is a special case.

Example 2.3.3. If $X^{\prime}$ is a noetherian algebraic space equipped with an action by a finite group $G$ and there is a $G$-invariant finite map $X^{\prime} \rightarrow S$ to a noetherian algebraic space $S$ then $X^{\prime} / G$ exists and the map $X^{\prime} / G \rightarrow S$ is proper and quasi-finite, hence finite.

Our proof of Proposition 2.3.1 will use the irreducible component decomposition for locally noetherian algebraic spaces, and we refer the reader to Proposition A.2.3 for a general discussion of this decomposition (avoiding the local separatedness hypotheses imposed in [K, II, §8.5]).

One final issue we address before taking up the proof of Proposition 2.3.1 is normalization in function field extensions for quasi-separated algebraic spaces. Let $X$ be a reduced and irreducible locally noetherian algebraic space (so $X$ is quasi-separated; see Definition A.2.1). Let $\eta$ be the unique generic point of $X$, so $X$ contains a dense open subspace around $\eta$ that is a scheme. The function field $k(X)$ is the henselian local ring of $X$ at $\eta$, or more concretely it is the common function field of any (necessarily reduced and irreducible) open scheme neighborhood of $\eta$ in $X$, so there is a canonical map Spec $k(X) \rightarrow X$.

By using an open scheme neighborhood of $\eta$ in $X$ we see that for any étale map $V \rightarrow X$ from a scheme, the pullback $V_{\eta}$ over Spec $k(X)$ is the scheme of generic points of $V$. Hence, for any finite reduced $k(X)$-algebra $K$, the pullback $V_{K}$ along Spec $K \rightarrow X$ is an étale $K$-scheme that is a finite flat cover of the scheme of generic points of $V$. The normalization $V^{\prime}$ of $V$ in $V_{K}$ therefore makes sense as an affine surjective map $V^{\prime} \rightarrow V$ that is finite when either $X$ is locally of finite type over an excellent scheme or $X$ is normal and $K / k(X)$ is separable, and the scheme of generic points of $V^{\prime}$ is identified with $V_{K}$.

The formation of the normalization $V^{\prime}$ is étale-local on $V$, so by étale descent the affine surjective maps $V^{\prime} \rightarrow V$ (for varying schemes $V$ étale over $X$ ) uniquely descend to a common affine surjective map of algebraic spaces $\pi: X^{\prime} \rightarrow X$. In particular, $X^{\prime}$ is normal and $\mathscr{O}_{X} \rightarrow \pi_{*}\left(\mathscr{O}_{X^{\prime}}\right)$ is injective. We call $X^{\prime} \rightarrow X$ the normalization of $X$ in $K / k(X)$. In the special case $K=k(X)$ we call $X^{\prime}$ the normalization of $X$. More generally, if $X$ is a reduced locally noetherian algebraic space that has finite many irreducible components $\left\{X_{i}\right\}$ then we can define the affine surjective normalization $X^{\prime} \rightarrow X$ of $X$ in any finite reduced faithfully flat algebra over $\prod k\left(X_{i}\right)$. 
Now assume that the reduced and irreducible locally noetherian $X$ is locally of finite type over an excellent scheme or that $X$ is normal and $K / k(X)$ is separable, so the normalization $\pi: X^{\prime} \rightarrow X$ is finite. By construction, the fiber $X_{\eta}^{\prime}=X^{\prime} \times_{X} \operatorname{Spec} k(X)$ is finite étale over $k(X)$ and it is identified with Spec $k\left(X_{i}^{\prime}\right)$, where $\left\{X_{i}^{\prime}\right\}$ is the finite set of irreducible components of $X^{\prime}$. (This is called the scheme of generic points of $X^{\prime}$.) The following lemma is a straightforward generalization (via étale descent) of its well-known analogue for schemes.

Lemma 2.3.4. Let $X$ be an irreducible and reduced locally noetherian algebraic space that is locally of finite type over an excellent scheme (resp. is normal), and let $\eta$ denote its unique generic point. Let $\mathscr{N}_{X}$ denote the category of finite (resp. finite generically étale) maps $f: X^{\prime} \rightarrow X$ from normal algebraic spaces $X^{\prime}$ such that $\mathscr{O}_{X} \rightarrow f_{*} \mathscr{O}_{X^{\prime}}$ is injective.

The functor $X^{\prime} \rightsquigarrow X_{\eta}^{\prime}$ is an equivalence from the category $\mathscr{N}_{X}$ to the category of non-empty finite (resp. non-empty finite generically étale) reduced $k(X)$-schemes, and normalization of $X$ in nonzero finite reduced (resp. nonzero finite étale) $k(X)$-algebras is a quasi-inverse.

Now we can give the proof of Proposition 2.3.1. By Proposition A.2.3, $X$ is irreducible. Let $\eta$ denote its unique generic point. Choose an étale covering $U \rightarrow X$ by an affine scheme, and let $L / k(X)$ be a finite Galois extension which splits the finite étale $k(X)$-scheme $U_{\eta}$. Let $\pi: X^{\prime} \rightarrow X$ denote the normalization of $X$ in $L$. Let $G=\operatorname{Gal}(L / k(X))$, so by the equivalence in Lemma 2.3.4 there is a natural right action by $G$ on $X^{\prime}$ over $X$. In particular, $G$ acts on the coherent $\mathscr{O}_{X}$-algebra $\pi_{*}\left(\mathscr{O}_{X^{\prime}}\right)$, so there is a natural injective map $\mathscr{O}_{X} \rightarrow \pi_{*}\left(\mathscr{O}_{X^{\prime}}\right)^{G}$ of coherent $\mathscr{O}_{X}$-algebras. We claim that this is an isomorphism. By normality it suffices to work over a Zariski-dense open subspace of $X$, so taking such a subspace that is an affine scheme does the job. Since $L / k(X)$ is Galois, we likewise see by working over such a dense open subscheme that $\pi$ is an étale $G$-torsor over a dense open subspace of $X$.

Since $X^{\prime} \rightarrow X$ is finite, by Example 2.3 .3 the quotient $X^{\prime} / G$ exists and the natural map $X^{\prime} / G \rightarrow X$ is finite. We can say more:

Lemma 2.3.5. The natural map $X^{\prime} / G \rightarrow X$ is an isomorphism.

Proof. The finite map $X^{\prime} \rightarrow X$ between irreducible noetherian algebraic spaces is dominant, so the same holds for $X^{\prime} / G \rightarrow X$. The algebraic space $X^{\prime} / G$ is also reduced since $X^{\prime}$ is reduced. The function field of $X^{\prime} / G$ contains $k(X)$ and is contained in $k\left(X^{\prime}\right)^{G}=L^{G}=k(X)$, so the finite map $X^{\prime} / G \rightarrow X$ is birational. It remains to use the fact that a finite birational map between reduced noetherian algebraic spaces is an isomorphism when the target is normal (as we may check by working étale-locally to reduce to the known case of schemes).

We have not yet used the precise way in which $L / k(X)$ was defined. This is essential to prove the next lemma, which will complete the proof of Proposition 2.3.1.

Lemma 2.3.6. The algebraic space $X^{\prime}$ is a scheme.

This assertion is [R1, Prop. 1], where a proof resting on Zariski's Main Theorem is given. We now give an alternative proof below for the convenience of the reader.

Proof. Recall that $L / k(X)$ is a finite Galois splitting field of the scheme of generic points Spec $A_{U}$ of an affine étale scheme cover $U \rightarrow X$. Consider the algebraic space $P=X^{\prime} \times_{X} U$. This is finite over $U$, so it is an affine scheme, and it is clearly a quasi-compact étale cover of $X^{\prime}$. In particular, $P$ is normal. Each connected component $P_{i}$ of $P$ maps birationally to $X^{\prime}$ since the scheme of generic points of $P$ is $\operatorname{Spec}\left(L \otimes_{k(X)} A_{U}\right) \simeq \coprod \operatorname{Spec} L$ due to $L / k(X)$ being a Galois splitting field for each factor field of $A_{U}$. We shall prove that each $P_{i}$ maps to $X^{\prime}$ via a monomorphism. Any étale monomorphism of algebraic spaces is an open immersion (as we deduce from the scheme case via descent), so it would follow that the étale covering map $P \rightarrow X^{\prime}$ realizes the $P_{i}$ 's as a collection of open subspaces that cover $X^{\prime}$ and are schemes, whence $X^{\prime}$ is a scheme as desired.

Now we show that each map $P_{i} \rightarrow X^{\prime}$ is a monomorphism, or in other words that the diagonal map $P_{i} \rightarrow P_{i} \times X^{\prime} P_{i}$ is an isomorphism. This diagonal is a closed immersion since $P_{i} \rightarrow X^{\prime}$ is separated (as the 
affine scheme $P_{i}$ is separated over $\operatorname{Spec} \mathbf{Z}$ ) and it is also étale, so it is an open immersion too. In other words, this diagonal realizes $P_{i}$ as a connected component of $P_{i} \times_{X^{\prime}} P_{i}$. But this fiber product has scheme of generic points $\operatorname{Spec}\left(L \otimes_{L} L\right)=\operatorname{Spec}(L)$ since $P_{i} \rightarrow X^{\prime}$ is étale, so $P_{i} \times_{X^{\prime}} P_{i}$ is irreducible. Therefore $\Delta_{P_{i} / X^{\prime}}$ is an isomorphism, as desired.

2.4. Proof of Theorem 1.2.1 when $X$ is normal. The aim of this section is to use the known Nagata compactification theorem for schemes (together with Proposition 2.3.1) to prove the following special case of Theorem 1.2.1, from which we will deduce the general case.

Theorem 2.4.1. Let $f: X \rightarrow S$ be a separated map of finite type between algebraic spaces, with $S$ of finite presentation over an excellent noetherian scheme and $X$ normal. Then $X$ admits an $S$-compactification; i.e., $f$ factors through an open immersion $j: X \rightarrow \bar{X}$ into an $S$-proper algebraic space.

Proof. Step 1. We first reduce to the case when $S$ is normal and both $X$ and $S$ are irreducible. The main subtlety is that irreducibility is not étale-local. We shall use the irreducible component decomposition of noetherian algebraic spaces; see Proposition A.2.3. We may replace $S$ with the schematic image of the separated finite type map $f: X \rightarrow S$, so $\mathscr{O}_{S} \rightarrow f_{*} \mathscr{O}_{X}$ is injective. Thus, $S$ is reduced and for each irreducible component $S_{j}$ of $S$, there is an irreducible component $X_{i(j)}$ of $X$ that is carried onto a dense subset of $S_{j}$ by $f$. In particular, the generic point of $X_{i(j)}$ is carried to the generic point of $S_{j}$. Writing $R_{X}$ and $R_{S}$ to denote the coordinate rings of the schemes of generic points, the preceding says exactly that $R_{S} \rightarrow R_{X}$ is a faithfully flat ring extension. This latter formulation has the advantage that (unlike irreducible components) it is compatible with passing to quasi-compact étale covers of $X$ and $S$.

Let the finite map $\widetilde{S} \rightarrow S$ denote the normalization of $S$ in its scheme of generic points (see Lemma 2.3.4 and the discussion preceding it). We claim that $f$ uniquely factors through a (necessarily separated, finite type, and schematically dominant) map $\tilde{f}: X \rightarrow \widetilde{S}$. This is well-known in the scheme case, and to handle the general case we use étale descent for morphisms: by the claimed uniqueness we may work étale-locally on $S$ to reduce to the case when it is a scheme, and we can then work over an étale scheme cover of $X$ to reduce to the case when $X$ is also a scheme.

By using $\widetilde{f}$, we may replace $S$ with $\widetilde{S}$ to reduce to the case when $S$ is normal. We may pass to connected components so that $X$ and $S$ are both connected and hence are irreducible.

Step 2. Now we make a digression, and prove Theorem 1.2.1 whenever $S$ is an excellent noetherian scheme and $X$ is arbitrary (e.g., not necessarily normal). This generality will be useful in Step 3. By Proposition 2.2.1 it suffices to treat the case when $X$ is normal. By Step 1 we may assume that both $X$ and $S$ are normal and connected. For the convenience of the reader, we explain the argument in terms of the theory of quotients that we reviewed in $\$ 2.3$ (resting on the use of quotient stacks).

By Proposition 2.3.1, there is a normal noetherian scheme $X^{\prime}$ equipped with a right action by a finite group $G$ and a $G$-invariant finite map $X^{\prime} \rightarrow X$ inducing an isomorphism $X^{\prime} / G \simeq X$. By Nagata's compactification theorem for schemes, there is an $S$-compactification $j: X^{\prime} \hookrightarrow \bar{X}^{\prime}$ with $\bar{X}^{\prime}$ a proper $S$-scheme. For each $g \in G$, let $j^{(g)}=j \circ[g]$ where $[g]: X^{\prime} \simeq X^{\prime}$ is the $S$-automorphism given by the right action by $g \in G$ on $X^{\prime}$. Thus, the fiber product $P=\prod_{g \in G} \bar{X}^{\prime}$ over $S$ is a proper $S$-scheme admitting a right $G$-action via $\left[g_{0}\right]:\left(\bar{x}_{g}^{\prime}\right)_{g} \mapsto\left(\bar{x}_{g_{0} g}^{\prime}\right)_{g}$ for varying $g_{0} \in G$. The map $X^{\prime} \rightarrow P$ defined by $x^{\prime} \mapsto\left(j^{(g)}\left(x^{\prime}\right)\right)_{g}$ is an immersion, and it is $G$-equivariant since $j^{\left(g_{0} g\right)}\left(x^{\prime}\right)=j\left(\left[g_{0} g\right]\left(x^{\prime}\right)\right)=j^{(g)}\left(\left[g_{0}\right]\left(x^{\prime}\right)\right)$. Hence, the schematic closure $\bar{X}_{1}^{\prime}$ of $X^{\prime}$ in $P$ is a $G$-equivariant compactification of $X^{\prime}$ over $S$.

Passing to quotient stacks, $\left[X^{\prime} / G\right] \rightarrow\left[\bar{X}_{1}^{\prime} / G\right]$ is an open immersion over $S$. Passing to the coarse moduli spaces, we get an $S$-map $X \simeq X^{\prime} / G \rightarrow \bar{X}_{1}^{\prime} / G$ with $\bar{X}_{1}^{\prime} / G$ proper over $S$. This is also an open immersion because the formation of coarse moduli spaces is compatible with passage to open substacks (as a special case of compatibility with quasi-separated flat base change on the coarse space). Hence, we have constructed an $S$-compactification of $X$ as an algebraic space. This proves Theorem 1.2.1 whenever $S$ is an excellent noetherian scheme.

Step 3. Finally, we return to the situation at the end of Step 1, so $S$ is a connected normal algebraic space of finite presentation over an excellent noetherian scheme and $X$ is normal and connected, hence generically 
flat over $S$. We seek to construct an $S$-compactification of $X$. The quotient technique that was used in Step 2 will be applied in order to reduce to the settled case when $S$ is a scheme (and $X$ is arbitrary).

Since $S$ is normal, by Proposition 2.3.1 we have $S \simeq S^{\prime} / G$ for some normal noetherian scheme $S^{\prime}$ equipped with a right action by a finite group $G$ and a finite surjective $G$-invariant map $S^{\prime} \rightarrow S$. Thus, $S^{\prime}$ is an excellent noetherian scheme. Let $X^{\prime}=\left(X \times_{S} S^{\prime}\right)_{\text {red }}$, so $X^{\prime}$ has a natural $G$-action over $X$ and $X^{\prime} \rightarrow X$ is a finite surjective $G$-invariant map. Since $X$ is generically flat over $S$, the induced finite map $X^{\prime} / G \rightarrow X$ is an isomorphism between dense opens, and thus is an isomorphism because $X$ is normal. Beware that $X^{\prime}$ may not be normal.

Since $S^{\prime}$ is an excellent noetherian scheme and in Step 2 we proved Theorem 1.2 .1 whenever the base is an excellent noetherian scheme, there is an $S^{\prime}$-compactification $j: X^{\prime} \hookrightarrow \bar{X}^{\prime}$. For each $g \in G$, let $[g]_{S^{\prime}}: S^{\prime} \simeq S^{\prime}$ and $[g]_{X^{\prime}}: X^{\prime} \simeq X^{\prime}$ denote the action maps for $g$ on $S^{\prime}$ and $X^{\prime}$ respectively (so $[g]_{X^{\prime}}$ is a map over $[g]_{S^{\prime}}$ ). Let $\bar{X}^{\prime(g)}=S^{\prime} \times_{[g]_{S^{\prime}}, S^{\prime}} \bar{X}^{\prime}$. Since $j \circ[g]_{X^{\prime}}: X^{\prime} \rightarrow \bar{X}^{\prime}$ is an open immersion over the automorphism $[g]_{S^{\prime}}$ of $S^{\prime}$, it induces an open immersion $j^{(g)}: X^{\prime} \hookrightarrow \bar{X}^{(g)}$ over $S^{\prime}$.

For the fiber product $P=\prod_{g \in G} \bar{X}^{\prime(g)}$ over $S^{\prime}$, any $g_{0} \in G$ induces an isomorphism $\bar{X}^{\prime(g)} \rightarrow \bar{X}^{\prime\left(g_{0}^{-1} g\right)}$ over $\left[g_{0}\right]_{S^{\prime}}$. For a fixed $g_{0} \in G$ these isomorphisms between the factors, all over a common automorphism of $S^{\prime}$, combine to define an automorphism of $P$ over the automorphism $\left[g_{0}\right]_{S^{\prime}}$ of $S^{\prime}$, and this is a right $G$-action on $P$ over the right $G$-action on $S^{\prime}$. Moreover, the immersion $X^{\prime} \rightarrow P$ defined by $x^{\prime} \mapsto\left(j^{(g)}(x)\right)$ is $G$-equivariant, exactly as in Step 2. Hence, the schematic closure $Z$ of $X^{\prime}$ in $P$ is an $S^{\prime}$-proper algebraic space equipped with a right $G$-action over the one on $S^{\prime}$, and this action is compatible with the given $G$-action on the open subscheme $X^{\prime}$. The induced $S$-map $\left[X^{\prime} / G\right] \rightarrow[Z / G]$ of Deligne-Mumford stacks is therefore an open immersion, so (exactly as in Step 2) it induces an open immersion of algebraic spaces $X \simeq X^{\prime} / G \hookrightarrow Z / G$ over $S$. Since $Z$ is $S^{\prime}$-proper, so is $Z / G \rightarrow S$.

2.5. Conclusion of the proof of Theorem 1.2.1. By Proposition 2.2.1 it is enough to prove Theorem 1.2.1 when $X$ is normal, and this is covered by Theorem 2.4.1.

\section{Approximation Results}

This section is devoted to establishing several general technical results which allow us to reduce problems to the noetherian (quasi-separated) case, and even to the case of algebraic spaces of finite presentation over Z. In particular, we will prove Theorem 1.2 .1 by using the settled case from $₫ 2$ with $S$ of finite presentation over $\mathbf{Z}$.

3.1. Absolute noetherian approximation. The key to avoiding noetherian hypotheses in Theorem 1.2.1 is the absolute noetherian approximation result in Theorem 1.2.2. We will prove Theorem 1.2 .2 by reducing it to the known case when $S$ is a scheme TT, Thms. C.7, C.9], in which case all $S_{\lambda}$ can be taken to be schemes. The reduction to the scheme case rests on the fact that any qcqs algebraic space admits a special kind of finite stratification by schemes:

Theorem 3.1.1 (Raynaud-Gruson). Let $S$ be a qcqs algebraic space. There is a finite rising chain

$$
\emptyset=U_{0} \subseteq U_{1} \subseteq \cdots \subseteq U_{r}=S
$$

of quasi-compact open subspaces such that for each $i>0$ the open subspace $U_{i}$ admits an étale cover $\varphi_{i}: Y_{i} \rightarrow$ $U_{i}$ by a quasi-compact separated scheme $Y_{i}$ with $\varphi_{i}$ restricting to an isomorphism over the closed subspace $Z_{i}=U_{i}-U_{i-1}$ in $U_{i}$ endowed with its reduced structure. Moreover, each $\varphi_{i}$ is separated, and each $Z_{i}$ is a separated and quasi-compact scheme.

Proof. The statement of [RG, I, 5.7.6] gives the existence result, except with étale covers $\varphi_{i}^{\prime}: Y_{i}^{\prime} \rightarrow\left(U_{i}\right)_{\text {red }}$ by quasi-compact separated schemes $Y_{i}^{\prime}$ such that each $\varphi_{i}^{\prime}$ restricts to an isomorphism over $Z_{i}$. The construction of $\varphi_{i}^{\prime}$ is as a pullback of an étale cover $\varphi_{i}: Y_{i} \rightarrow U_{i}$ by a quasi-compact separated scheme $Y_{i}$. The map $\varphi_{i}$ is necessarily separated since the composition of $\Delta_{\varphi_{i}}$ with the monomorphism $Y_{i} \times_{U_{i}} Y_{i} \rightarrow Y_{i} \times_{\operatorname{Spec}} \mathbf{Z} Y_{i}$ is a closed immersion. 
Definition 3.1.2. Let $\Lambda$ be a noetherian ring and $S$ an algebraic space over $\Lambda$. We say that $S$ is $\Lambda$ approximable if there is a $\Lambda$-isomorphism $S \simeq \lim S_{\alpha}$ where $\left\{S_{\alpha}\right\}$ is an inverse system of algebraic spaces of finite presentation over $\Lambda$ having affine transition maps $S_{\beta} \rightarrow S_{\alpha}$ for all $\alpha$ and all $\beta \geq \alpha$. In case $\Lambda=\mathbf{Z}$, we say that $S$ is approximable.

Observe that we use "finite presentation" rather than just "finite type" in Definition 3.1.2. This is essential, as we indicated in $\$ 1.3$. Any inverse limit as in Definition 3.1 .2 is necessarily qcqs (over $\Lambda$, or equivalently over $\mathbf{Z}$ ), and our aim is to prove that conversely every qcqs algebraic space over $\Lambda$ is $\Lambda$-approximable. The most interesting case is $\Lambda=\mathbf{Z}$, and in fact this is enough to settle the general case:

Lemma 3.1.3. Let $\Lambda$ be a noetherian ring, and $S$ a $\Lambda$-approximable algebraic space. The inverse system $\left\{S_{\alpha}\right\}$ as in Definition 3.1.2 can be taken to have schematically dominant affine transition maps. Moreover, if $\Lambda \rightarrow \Lambda^{\prime}$ is a map of noetherian rings and $S$ admits a compatible structure of algebraic space over $\Lambda^{\prime}$ then $S$ is also $\Lambda^{\prime}$-approximable.

Proof. Choose an inverse system $\left\{S_{\alpha}\right\}$ of algebraic spaces of finite presentation over $\Lambda$ with affine transition maps such that $S \simeq \lim S_{\alpha}$ over $\Lambda$. Each map $q_{\alpha}: S \rightarrow S_{\alpha}$ is affine, so it admits a scheme-theoretic image $S_{\alpha}^{\prime} \subseteq S_{\alpha}$ that is the closed subspace corresponding to the quasi-coherent kernel of $\mathscr{O}_{S_{\alpha}} \rightarrow q_{\alpha *}\left(\mathscr{O}_{S}\right)$. By working étale-locally over a fixed $S_{\alpha_{0}}$ we see that the map $q^{\prime}: S \rightarrow \lim _{\longleftarrow} S_{\alpha}^{\prime}$ is an isomorphism and $q_{\alpha}^{\prime}: S \rightarrow S_{\alpha}^{\prime}$ and $q_{\alpha \beta}^{\prime}: S_{\beta}^{\prime} \rightarrow S_{\alpha}^{\prime}$ are schematically dominant and affine for all $\alpha$ and all $\beta \geq \alpha$.

Now assume there is given a (necessarily quasi-separated) $\Lambda$-morphism $S \rightarrow \operatorname{Spec} \Lambda^{\prime}$ for a noetherian $\Lambda$-algebra $\Lambda^{\prime}$. Fix $\alpha_{0}$ and define the quasi-coherent sheaf

$$
\mathscr{A}_{\alpha}:=\Lambda^{\prime} \cdot q_{\alpha_{0}, \alpha *}^{\prime}\left(\mathscr{O}_{S_{\alpha}^{\prime}}\right) \subseteq q_{\alpha_{0} *}^{\prime}\left(\mathscr{O}_{S}\right)
$$

of $\mathscr{O}_{S_{\alpha_{0}}}$-algebras for $\alpha \geq \alpha_{0}$. The algebraic spaces $S_{\alpha}^{\prime \prime}=\operatorname{Spec}_{S_{\alpha_{0}}^{\prime}}\left(\mathscr{A}_{\alpha}\right)$ of finite presentation over $\Lambda^{\prime}$ form an inverse system with schematically dominant and affine transition maps such that $\lim _{\alpha}^{\prime \prime} \simeq S$ over $\Lambda^{\prime}$.

To make effective use of Theorem 3.1.1 in the proof that $S$ is approximable, the idea is to use induction on $r$ to get to the situation of extending an inverse system approximation for $U_{r-1}$ across the complementary scheme $Z_{r}$ to get an inverse system approximation for $S$. Making such an extension requires studying the algebraic spaces $U_{r-1}$ and $S$ in terms of étale scheme presentations, which must themselves be replaced with compatible inverse limit approximations. The strategy is to reconstruct $S$ as a kind of pushout of $Z_{r}$ against an étale scheme chart of $U_{r-1}$, but everything must be done at the level of inverse systems. Moreover, the constructions we give have to be sufficiently "cartesian" so that we can compute what happens in the limit. This leads us to the following initial lemma in which a cartesian property at the level of étale scheme charts implies a cartesian property for the quotients. It will be more convenient to work with sheaf-functors instead of schemes:

Lemma 3.1.4. Let $R \rightrightarrows U$ and $R^{\prime} \rightrightarrows U^{\prime}$ be equivalence relations in sheaves of sets on the étale site of the category of schemes. Assume that there is given a map $f: U^{\prime} \rightarrow U$ such that $f \times f: U^{\prime} \times U^{\prime} \rightarrow U \times U$ carries $R^{\prime}$ into $R$ and the co-commutative diagram

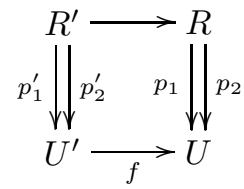

is cartesian for each pair $\left(p_{i}, p_{i}^{\prime}\right)$. Then the induced commutative square

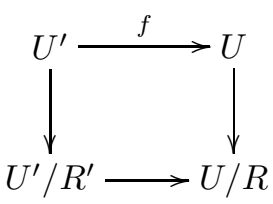

is cartesian. 
Proof. We have to show that the natural map $U^{\prime} \rightarrow\left(U^{\prime} / R^{\prime}\right) \times_{U / R} U$ is an isomorphism as étale sheaves on any scheme $T$. It suffices to check on stalks, which is to say on $T$-valued points at geometric points $\bar{t}:$ Spec $k \rightarrow T$, with $k$ a separable closure of the residue field at an arbitrary point $t \in T$. Hence, $(U / R)_{\bar{t}}=U_{\bar{t}} / R_{\bar{t}}$ and $\left(U^{\prime} / R^{\prime}\right)_{\bar{t}}=U_{\bar{t}}^{\prime} / R_{\bar{t}}^{\prime}$, so we need to prove that the natural map

$$
U_{\bar{t}}^{\prime} \rightarrow\left(U_{\bar{t}}^{\prime} / R_{\bar{t}}^{\prime}\right) \times_{U_{\bar{t}} / R_{\bar{t}}} U_{\bar{t}}
$$

is bijective. We will do this using the commutative diagram

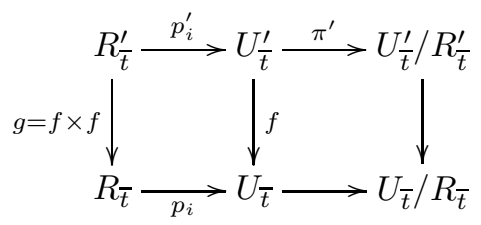

with cartesian left square for $i \in\{1,2\}$.

To prove surjectivity of (3.1.4.1), choose $\alpha \in\left(U_{\bar{t}}^{\prime} / R_{\bar{t}}^{\prime}\right) \times_{U_{\bar{t}} / R_{\bar{t}}} U_{\bar{t}}$, so $\alpha=\left(u^{\prime} \bmod R_{\bar{t}}^{\prime}, u\right)$ for some $u^{\prime} \in U_{\bar{t}}^{\prime}$ and $u \in U_{\bar{t}}$. The fiber product condition on $\alpha$ says $\left(f\left(u^{\prime}\right), u\right) \in R_{\bar{t}}$ inside $U_{\bar{t}} \times U_{\bar{t}}$. The cartesian property of the left square in (3.1.4.2) with $i=1$ therefore gives a unique point $r^{\prime} \in R_{\bar{t}}^{\prime}$ with $g\left(r^{\prime}\right)=\left(f\left(u^{\prime}\right), u\right)$ and $p_{1}^{\prime}\left(r^{\prime}\right)=u^{\prime}$. The commutativity of the left square with $i=2$ says that if $y^{\prime}=p_{2}^{\prime}\left(r^{\prime}\right)$ then $f\left(y^{\prime}\right)=u$. Hence, (3.1.4.1) carries $y^{\prime} \in U_{\bar{t}}^{\prime}$ over to $\left(y^{\prime} \bmod R_{\bar{t}}^{\prime}, u\right)=\left(u^{\prime} \bmod R_{\bar{t}}^{\prime}, u\right)=\alpha$.

Now pick $u_{1}^{\prime}, u_{2}^{\prime} \in U_{\bar{t}}^{\prime}$ that are carried to the same point under (3.1.4.1), which is to say that $\left(u_{1}^{\prime}, u_{2}^{\prime}\right) \in R_{\bar{t}}^{\prime}$ and $f\left(u_{1}^{\prime}\right)=f\left(u_{2}^{\prime}\right)$ in $U_{\bar{t}}$. Letting $r^{\prime}=\left(u_{1}^{\prime}, u_{2}^{\prime}\right)$, clearly $g\left(r^{\prime}\right)=\left(f\left(u_{1}^{\prime}\right), f\left(u_{2}^{\prime}\right)\right)=\left(f\left(u_{1}^{\prime}\right), f\left(u_{1}^{\prime}\right)\right)$. That is, $g\left(r^{\prime}\right)=\Delta\left(f\left(u_{1}^{\prime}\right)\right)$ where $\Delta: U \rightarrow R$ is the diagonal section. But the point $\Delta\left(u_{1}^{\prime}\right) \in R_{\bar{t}}^{\prime}$ satisfies

$$
g\left(\Delta\left(u_{1}^{\prime}\right)\right)=\left(f\left(u_{1}^{\prime}\right), f\left(u_{1}^{\prime}\right)\right)=g\left(r^{\prime}\right), p_{1}^{\prime}\left(\Delta\left(u_{1}^{\prime}\right)\right)=u_{1}^{\prime}=p_{1}^{\prime}\left(r^{\prime}\right),
$$

so the cartesian property of the left square in (3.1.4.2) for $i=1$ implies that $r^{\prime}=\Delta\left(u_{1}^{\prime}\right)=\left(u_{1}^{\prime}, u_{1}^{\prime}\right)$. Since $r^{\prime}=\left(u_{1}^{\prime}, u_{2}^{\prime}\right)$ by definition, we get $u_{2}^{\prime}=u_{1}^{\prime}$ as required for injectivity of (3.1.4.1).

Corollary 3.1.5. In the setup of Lemma 3.1.4, if $U, U^{\prime}, R$, and $R^{\prime}$ are algebraic spaces with maps $p_{i}$ and $p_{i}^{\prime}$ étale, and if $U^{\prime} \rightarrow U$ satisfies a property $\mathbf{P}$ of morphisms of algebraic spaces that is étale-local on the base, then $U^{\prime} / R^{\prime} \rightarrow U / R$ satisfies property $\mathbf{P}$.

By Corollary A.1.2 $U / R$ and $U^{\prime} / R^{\prime}$ are algebraic spaces.

Proof. To analyze the asserted property of $U^{\prime} / R^{\prime} \rightarrow U / R$ it suffices to check after pullback to the étale covering $U$ of $U / R$. By Lemma 3.1.4, this pullback is identified with the map $U^{\prime} \rightarrow U$.

To apply Corollary 3.1.5 we wish to describe a situation in which the setup of Lemma 3.1.4 naturally arises. We first require one further lemma, concerning the existence and properties of certain pushouts.

Lemma 3.1.6. Consider a diagram of algebraic spaces

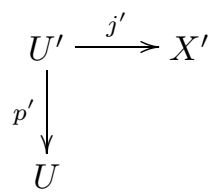

in which $j^{\prime}$ is an open immersion and $p^{\prime}$ is an étale surjection.

(1) There exists a pushout $X=U \coprod_{U^{\prime}} X^{\prime}$ in the category of algebraic spaces, and the associated diagram

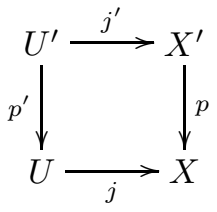


is cartesian, with $j$ an open immersion and $p$ an étale surjection. The formation of this pushout commutes with any base change on $X$ in the sense that if $X_{1} \rightarrow X$ is any map of algebraic spaces then the pullback diagram

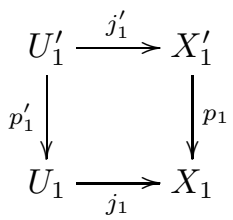

is also a pushout.

(2) If $j^{\prime}$ is quasi-compact then $j$ is quasi-compact, if $p^{\prime}$ is separated then $p$ is separated, and if $j^{\prime}$ is quasi-compact and $p^{\prime}$ is finitely presented then $p$ is also finitely presented.

(3) If $U, U^{\prime}$, and $X^{\prime}$ are qcqs then so is $X$.

Note that in (1), the asserted compatibility with any base change is only being made for the kinds of pushouts considered there, namely pushout of an étale surjection along an open immersion. This is a mild (but useful) generalization of the familiar base change compatibility of a Zariski gluing, which can be viewed as a pushout of one open immersion along another.

Proof. Since $\Delta_{p^{\prime}}: U^{\prime} \rightarrow U^{\prime} \times_{U} U^{\prime}$ is a section to an étale map of algebraic spaces, it is an étale map. Thus, $\Delta_{p^{\prime}}$ is an étale monomorphism. An étale monomorphism of algebraic spaces is always an open immersion. Indeed, in the special case of quasi-separated algebraic spaces we may work Zariski-locally to reduce to the finitely presented case, which is [K, II, Lemma 6.15b]. This handles the general case when the target is affine, as then the source is separated (due to separatedness of monomorphisms). In general, we may work étale-locally on the base to reduce to the settled case when the target is affine.

Since $\Delta_{p_{1}}$ is an open immersion, it makes sense to form the gluing

$$
R=X^{\prime} \coprod_{U^{\prime}}\left(U^{\prime} \times_{U} U^{\prime}\right)
$$

of $X^{\prime}$ and $U^{\prime} \times_{U} U^{\prime}$ along the common open subspace $U^{\prime}$. Using the maps $U^{\prime} \times_{U} U^{\prime} \rightrightarrows U^{\prime} \stackrel{j^{\prime}}{\hookrightarrow} X^{\prime}$, we arrive at natural maps $R \rightrightarrows X^{\prime}$ that clearly constitute an étale equivalence relation in algebraic spaces. The quotient $X:=X^{\prime} / R$ is an algebraic space, and by construction $p: X^{\prime} \rightarrow X$ is an étale surjection. By the definition of $R$, the map $j^{\prime}: U^{\prime} \hookrightarrow X^{\prime}$ induces a canonical map

$$
j: U=U^{\prime} /\left(U^{\prime} \times_{U} U^{\prime}\right) \rightarrow X^{\prime} / R=X,
$$

so we obtain the commutative diagram (3.1.6.1). By using the definition of $R$, this diagram is easily checked to be cartesian and a pushout. Due to the cartesian property and the fact that $p$ is an étale surjection, $j$ is an open immersion because $j^{\prime}$ is an open immersion.

To complete the proof of (1), we have to verify the compatibility with base change on $X$. Letting $X_{1} \rightarrow X$ be a map from an algebraic space, we have to prove that (3.1.6.2) is a pushout. That is, we want the natural $X_{1}$-map $U_{1} \coprod_{U_{1}^{\prime}} X_{1}^{\prime} \rightarrow X_{1}$ to be an isomorphism. In view of the construction of this latter pushout as a quotient, it is equivalent to say that the map $p_{1}: X_{1}^{\prime} \rightarrow X_{1}$ is an étale cover and

$$
h_{1}: R_{1}=X_{1}^{\prime} \coprod_{U_{1}^{\prime}}\left(U_{1}^{\prime} \times_{U_{1}} U_{1}^{\prime}\right) \rightarrow X_{1}^{\prime} \times_{X_{1}} X_{1}^{\prime}
$$

is an isomorphism, where $R_{1}$ is a gluing for the Zariski topology.

The map $p_{1}$ is a base change of $p$, so it is an étale surjection. To prove that $h_{1}$ is an isomorphism, we observe that $X_{1}^{\prime} \times_{X_{1}} X_{1}^{\prime}=\left(X^{\prime} \times_{X} X^{\prime}\right) \times_{X} X_{1}$ and likewise $R_{1}=R \times_{X} X_{1}$ because the formation of Zariski gluings of objects over a common base (such as $X^{\prime}$ and $U^{\prime} \times_{U} U^{\prime}$ glued along the open $U^{\prime}$, all over the base $X)$ commutes with any base change. Hence, $h_{1}$ is identified with the base change along $X_{1} \rightarrow X$ of the analogous map $h: R \rightarrow X^{\prime} \times_{X} X^{\prime}$ that is an isomorphism (because $X:=X^{\prime} / R$ ). This completes the proof of $(1)$. 
Consider the claims in (2). By descent through $p$, the map $j$ is quasi-compact when $j^{\prime}$ is quasi-compact. Assuming that $p^{\prime}$ is separated, the open subspace $U^{\prime}$ in $U^{\prime} \times_{U} U^{\prime}$ via the diagonal is also closed and hence splits off as a disjoint union: $U^{\prime} \times_{U} U^{\prime}=\Delta\left(U^{\prime}\right) \coprod V$ for an algebraic space $V$ that is separated over $U^{\prime}$ (and hence over $X^{\prime}$ ) via either projection. Thus, in such cases $R=X^{\prime} \amalg V$ is separated over $X^{\prime}$ via either projection, so $p: X^{\prime} \rightarrow X$ is separated. In case $j^{\prime}$ is quasi-compact (so $U^{\prime} \rightarrow X^{\prime}$ is finitely presented) and $p^{\prime}$ is finitely presented, the map $p: X^{\prime} \rightarrow X$ is finitely presented because it is a descent of either of the projection maps $R \rightrightarrows X^{\prime}$ which express $R$ as a gluing of two finitely presented $X^{\prime}$-spaces along a common finitely presented open subspace.

Finally, to prove (3), observe that (by construction) if $U, U^{\prime}$, and $X^{\prime}$ are qcqs then $R$ is qcqs, so the maps $R \rightrightarrows X^{\prime}$ are qcqs and hence the quotient map $X^{\prime} \rightarrow X$ is qcqs. Thus, in such cases $X$ is qcqs.

Notation 3.1.7. We will sometimes refer to the étale equivalence relation $X^{\prime} \times_{X} X^{\prime} \rightrightarrows X^{\prime}$ constructed in Lemma 3.1.6 as being obtained from the étale equivalence relation $U^{\prime} \times_{U} U^{\prime} \rightrightarrows U$ via extension along the diagonal.

To prove Theorem 1.2.1, we wish to inductively construct limit presentations of qcqs algebraic spaces by means of stratifications as in Theorem 3.1.1. This will be achieved by using the following result.

Proposition 3.1.8. Let $X$ be a qcqs algebraic space, and suppose there is given a diagram

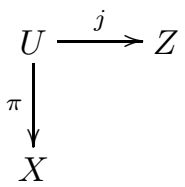

in which $\pi$ is a finitely presented étale scheme covering and $j$ is an open immersion into a qcqs scheme $Z$. Form the cartesian pushout diagram in algebraic spaces

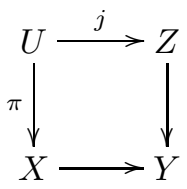

as in Lemma 3.1.6, so the bottom side is an open immersion, the right side is an étale surjection, and $Y$ is qcqs.

If $X$ is approximable then so is $Y$.

To prove Proposition 3.1.8, we first need to study pairs of diagrams of the type in (3.1.8.1) that are connected to each other via affine and schematically dominant maps. Thus, we now briefly digress to consider such diagrams and their corresponding pushouts as in Lemma 3.1.6.

Let $X^{\prime} \rightarrow X$ be an affine and schematically dominant map of algebraic spaces, and let $U \rightarrow X$ be an étale covering by a scheme, so $h: U^{\prime}:=U \times_{X} X^{\prime} \rightarrow U$ is affine (hence $U^{\prime}$ is a scheme) and $U^{\prime} \rightarrow X^{\prime}$ is an étale covering. Note that the affine map $U^{\prime} \rightarrow U$ is schematically dominant. Suppose that there is a cartesian square of schemes

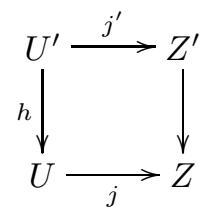

in which the horizontal maps are open immersions and the right vertical map is affine (like the left side).

The respective algebraic space quotients $X$ and $X^{\prime}$ of $U$ and $U^{\prime}$ give rise to étale equivalence relations in schemes

$$
U \times{ }_{X} U \rightrightarrows U, U^{\prime} \times_{X^{\prime}} U^{\prime} \rightrightarrows U^{\prime}
$$


and we extend these to étale equivalence relations in schemes

$$
R \rightrightarrows Z, \quad R^{\prime} \rightrightarrows Z^{\prime}
$$

via extension along the diagonal, exactly as in the proof of Lemma 3.1.6] define the subfunctors $R \subseteq Z \times Z$ and $R^{\prime} \subseteq Z^{\prime} \times Z^{\prime}$ to respectively be the gluings along common open subspaces

$$
R=\Delta(Z) \coprod_{\Delta(U)}\left(U \times_{X} U\right), R^{\prime}=\Delta\left(Z^{\prime}\right) \coprod_{\Delta^{\prime}\left(U^{\prime}\right)}\left(U^{\prime} \times_{X^{\prime}} U^{\prime}\right) .
$$

In particular, the diagrams
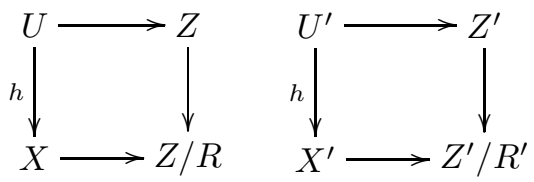

are cartesian and are pushouts with open immersions along the bottom, and if (as in applications below) $X$, $U$, and $Z$ are qcqs (so likewise for $X^{\prime}, U^{\prime}$, and $Z^{\prime}$ ) then $R, R^{\prime}, Z / R$, and $Z^{\prime} / R^{\prime}$ are qcqs.

Corollary 3.1.9. In the above situation, the co-commutative diagram

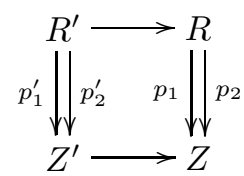

is cartesian for each pair $\left(p_{i}, p_{i}^{\prime}\right)$, and the map of pushouts $Z^{\prime} / R^{\prime} \rightarrow Z / R$ is affine and schematically dominant.

Proof. It is straightforward to check that the cartesian property holds, using that $U^{\prime}=U \times_{X} X^{\prime}$ by definition. Thus, the hypotheses of Lemma 3.1.4 are satisfied. By Corollary 3.1.5 we are done.

The reason for our interest in Corollary 3.1 .9 is that it arises in the proof of Proposition 3.1 .8 , which we now give:

Proof of Prop. 3.1.8. Since $X$ is approximable, we may choose an isomorphism $X \simeq \lim X_{\alpha}$ with $\left\{X_{\alpha}\right\}$ an inverse system of algebraic spaces of finite presentation over $\mathbf{Z}$ with affine transition maps. We may and do arrange that the transition maps are also schematically dominant. By Proposition A.3.4. we may also assume (by requiring $\alpha$ to be sufficiently large) that this isomorphism is covered by an isomorphism $U \simeq \lim U_{\alpha}$ where $\left\{U_{\alpha}\right\}$ is an inverse system of finitely presented schemes over $\left\{X_{\alpha}\right\}$ such that the maps $U_{\beta} \rightarrow X_{\beta} \times_{X_{\alpha}} U_{\alpha}$ are isomorphisms whenever $\beta \geq \alpha$. (In particular, $\left\{U_{\alpha}\right\}$ has affine transition maps, so $\lim _{\alpha} U_{\alpha}$ makes sense.)

By Corollary A.3.5 we may and do require $\alpha$ to be sufficiently large so that the finitely presented maps $h_{\alpha}: U_{\alpha} \rightarrow X_{\alpha}$ are étale coverings. Thus, by flatness of $h_{\alpha}$, the inverse system $\left\{U_{\alpha}\right\}$ has schematically dominant transition maps since the same holds for $\left\{X_{\alpha}\right\}$. Moreover, each scheme $U_{\alpha}$ is of finite type over $\mathbf{Z}$ since $X_{\alpha}$ is of finite presentation over $\mathbf{Z}$ (and $h_{\alpha}$ is finitely presented). Hence, by applying Lemma A.3.6 to the quasi-compact open immersion $U \hookrightarrow Z$, at the expense of possibly modifying the indexing system we can arrange that there is a cartesian inverse system of quasi-compact open immersions $j_{\alpha}: U_{\alpha} \hookrightarrow Z_{\alpha}$ of finite type $\mathbf{Z}$-schemes such that $\left\{Z_{\alpha}\right\}$ has affine and schematically dominant transition maps and lim $j_{\alpha}$ is the given open immersion $j: U \hookrightarrow Z$. We emphasize that it is the application of Lemma A.3.6 that is the entire reason we had to make the affine transition maps in our initial inverse systems be schematically dominant.

Consider the system of diagrams

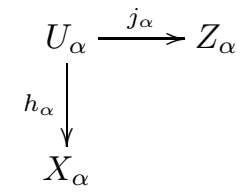


in which the maps on the left are étale scheme coverings. This is a "cartesian" system of diagrams in the sense that the diagrams

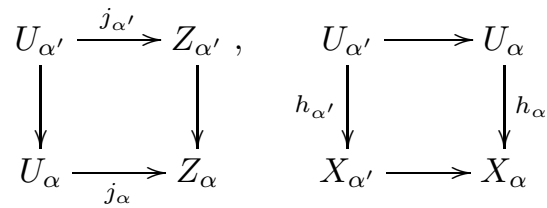

for $\alpha^{\prime} \geq \alpha$ are cartesian. Thus, the setup preceding Corollary 3.1.9 is applicable to the system of étale scheme coverings $h_{\alpha}: U_{\alpha} \rightarrow X_{\alpha}$ with affine and schematically dominant transition maps, equipped with the compatible open immersions $j_{\alpha}: U_{\alpha} \hookrightarrow Z_{\alpha}$.

By Corollary 3.1.9 we thereby obtain a cartesian system of étale equivalence relations $R_{\alpha} \rightrightarrows Z_{\alpha}$ in qcqs schemes akin to (3.1.8.3) and the resulting qcqs algebraic space quotients $Y_{\alpha}=Z_{\alpha} / R_{\alpha}$ naturally fit into an inverse system with affine and schematically dominant transition maps. These quotients are exactly the pushouts $X_{\alpha} \bigsqcup_{U_{\alpha}} Z_{\alpha}$ as constructed in Lemma 3.1.6. Each $Y_{\alpha}$ is of finite type over $\mathbf{Z}$ since the same holds for its étale scheme covering $Z_{\alpha}$, so each $Y_{\alpha}$ is finitely presented over $\mathbf{Z}$ (as each $Y_{\alpha}$ is quasi-separated).

Consider the pushout diagram

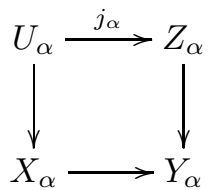

of algebraic spaces as in (3.1.6.1), so these are cartesian and have $X_{\alpha} \rightarrow Y_{\alpha}$ an open immersion. For any $\alpha^{\prime} \geq \alpha$, the diagram (3.1.9.3) for $\alpha^{\prime}$ maps to the one for $\alpha$, and we claim that this resulting inverse system of diagrams is a cartesian system in the sense that the pullback of (3.1.9.3) along $Y_{\alpha^{\prime}} \rightarrow Y_{\alpha}$ is identified (via the natural maps) with the $\alpha^{\prime}$-version of (3.1.9.3). This cartesian system claim along the right side of the diagrams (3.1.9.3) is Lemma 3.1.4, and along the top and left sides it is the cartesian property observed in (3.1.9.2). Since $X_{\alpha}$ is the image of the open subspace $U_{\alpha} \hookrightarrow Z_{\alpha}$ along the étale quotient map $Z_{\alpha} \rightarrow Y_{\alpha}$, and likewise for $\alpha^{\prime} \geq \alpha$, the cartesian property along the bottom sides of the diagrams (3.1.9.3) follows.

The definition of $Y$ as a pushout provides maps $Y \rightarrow Y_{\alpha}$ respecting change in $\alpha$, and we shall prove that the induced map $Y \rightarrow \lim Y_{\alpha}$ is an isomorphism. This would show that $Y$ is approximable, as desired.

Define $R=\lim R_{\alpha} \subseteq \lim \left(Z_{\alpha} \times_{\operatorname{Spec} \mathbf{Z}} Z_{\alpha}\right)=Z \times_{\operatorname{Spec} \mathbf{Z}} Z$, so $R$ is a qcqs scheme and the pair of maps $p_{1}, p_{2}: R \rightrightarrows Z$ obtained from passage to the limit on the cartesian system $p_{1, \alpha}, p_{2, \alpha}: R_{\alpha} \rightrightarrows Z_{\alpha}$ is an étale equivalence relation. Lemma 3.1.4 ensures that the natural maps $Z_{\beta} \rightarrow Z_{\alpha} \times_{Y_{\alpha}} Y_{\beta}$ are isomorphisms for all $\beta \geq \alpha$, so passing to the limit on $\beta$ with a fixed $\alpha$ gives that the natural map $Z \rightarrow Z_{\alpha} \times_{Y_{\alpha}} Y$ is an isomorphism (since inverse limits of algebraic spaces under affine transition maps commute with fiber products). Similarly, for each fixed $i \in\{1,2\}$ and $\beta \geq \alpha$, the natural map $R_{\beta} \rightarrow R_{\alpha} \times_{p_{i, \alpha}, Z_{\alpha}} Z_{\beta}$ over $p_{i, \beta}: R_{\beta} \rightarrow Z_{\beta}$ is an isomorphism due to the cartesian observation preceding Corollary 3.1.9. Hence, passing to the limit on $\beta$ with a fixed $\alpha$ gives that the natural map $R \rightarrow R_{\alpha} \times_{p_{i, \alpha}, Z_{\alpha}} Z$ over $p_{i}: R \rightarrow Z$ is an isomorphism for all $\alpha$. But $R_{\alpha}=Z_{\alpha} \times_{Y_{\alpha}} Z_{\alpha}$, so taking $i=2$ gives

$$
R \simeq Z_{\alpha} \times_{Y_{\alpha}} Z
$$

for all $\alpha$. Hence, passing to the limit on $\alpha$ gives $R=Z \times_{Y} Z$. In other words, $R \rightrightarrows Z$ is an étale chart in qcqs schemes for the algebraic space $Y$.

Our problem is now reduced to showing that the natural map of algebraic spaces $\phi: Z / R \rightarrow \lim \left(Z_{\alpha} / R_{\alpha}\right)$ is an isomorphism, where the inverse system of algebraic spaces $\left\{Z_{\alpha} / R_{\alpha}\right\}=\left\{Y_{\alpha}\right\}$ has affine and schematically dominant transition maps. The map $\phi$ is affine and schematically dominant since Corollary 3.1.9 implies that each map $Z / R \rightarrow Z_{\alpha} / R_{\alpha}$ is affine and schematically dominant. But the qcqs étale coverings $Z_{\alpha} \rightarrow$ $Y_{\alpha}=Z_{\alpha} / R_{\alpha}$ are cartesian with respect to change in $\alpha$, so passing to the limit gives that $Z=\lim Z_{\alpha}$ is a qcqs étale scheme cover of $\lim _{(}\left(Z_{\alpha} / R_{\alpha}\right)$. This covering by $Z$ is compatible with $\phi$, so the affine map $\phi$ is an étale surjection. Since $R=\lim _{\longleftarrow} R_{\alpha}$ inside of $Z \times Z=\lim _{\longleftarrow}\left(Z_{\alpha} \times Z_{\alpha}\right)$, it follows that $\phi$ is a monomorphism. 
Being affine and étale, it is therefore also a quasi-compact open immersion. But $\phi$ is an étale cover, so it is an isomorphism.

To apply Proposition 3.1.8 repeatedly in the context of Theorem 3.1.1 we require one more lemma.

Lemma 3.1.10. Let $S$ be a qcqs algebraic space, and choose a finite rising chain $\left\{U_{i}\right\}$ of quasi-compact open subspaces in $S$ and quasi-compact étale scheme covers $\varphi_{i}: Y_{i} \rightarrow U_{i}$ with separated $Y_{i}$ as in Theorem 3.1.1. For each $i>0$, consider the diagram of cartesian squares

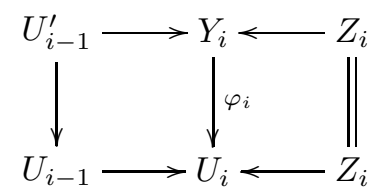

where $Z_{i}:=U_{i}-U_{i-1}$ endowed with the reduced structure.

The étale equivalence relation in schemes $R_{i}=Y_{i} \times_{U_{i}} Y_{i} \rightrightarrows Y_{i}$ is the extension along the diagonal (in the sense of Lemma (3.1.6) of the étale equivalence relation $U_{i-1}^{\prime} \times_{U_{i-1}} U_{i-1}^{\prime} \rightrightarrows U_{i-1}^{\prime}$.

Proof. The subfunctor $R_{i} \subseteq Y_{i} \times Y_{i}$ contains the subfunctors $\Delta\left(Y_{i}\right)$ and $U_{i-1}^{\prime} \times_{U_{i-1}} U_{i-1}^{\prime}$ which overlap along the common open subfunctor $\Delta\left(U_{i-1}^{\prime}\right)$ (openness in $U_{i-1}^{\prime} \times_{U_{i-1}} U_{i-1}^{\prime}$ due to $U_{i-1}^{\prime} \rightarrow U_{i-1}$ being étale). Our aim is to prove that the inclusion

$$
\eta_{i}: \Delta\left(Y_{i}\right) \coprod_{\Delta\left(U_{i-1}^{\prime}\right)}\left(U_{i-1}^{\prime} \times_{U_{i-1}} U_{i-1}^{\prime}\right) \subseteq R_{i}
$$

between subfunctors of $Y_{i} \times Y_{i}$ is an isomorphism. Restricting over the open subscheme $U_{i-1}^{\prime} \times U_{i-1}^{\prime}$ clearly gives an isomorphism, and since $\varphi_{i}$ is étale and separated we see that $\Delta\left(Y_{i}\right)$ is an open and closed subscheme of $R_{i}$. Thus, $\eta_{i}$ is an open immersion of schemes, so it suffices to check equality on geometric fibers over $U_{i}$. Over $U_{i-1}$ the situation is clear, and over $U_{i}-U_{i-1}=Z_{i}$ it is also clear since $\varphi_{i}$ restricts to an isomorphism over $Z_{i}$ (so the part of $R_{i}$ lying over $Z_{i} \subseteq U_{i}$ is contained in $\Delta\left(Y_{i}\right)$ on geometric points).

Now we are finally in position to prove Theorem 1.2 .2

Proof of Theorem 1.2.2. Fix a stratification and associated étale coverings as in Theorem 3.1.1. We shall prove Theorem 1.2.2 by induction on $r$, the case $r=0$ being the trivial case of empty $S$. In general, by induction we may assume $r \geq 1$ and that $U_{r-1}$ is approximable. (Note that if $r=1$ then $S_{\text {red }}$ is a scheme, but we do not yet know that this forces $S$ to be a scheme in general. Hence, approximation for schemes [TT, Thm. C.9] does not suffices to settle the case $r=1$.) By Lemma 3.1.10 the open immersion $U_{r-1} \hookrightarrow U_{r}=S$ arises along the bottom side of a pushout diagram as in (3.1.8.2). Thus, by Proposition 3.1.8 the approximability of $S$ follows from that of $U_{r-1}$. This completes the proof.

Remark 3.1.11. In case the reader is wondering where the scheme case of Theorem 1.2.2(i.e., [TT, Thm. C.9]) is lurking in our proof for the case of algebraic spaces, it is used in the proof of Lemma A.3.3, which in turn is an essential ingredient in proofs of subsequent results in A.3 that were used in our treatment of the case of algebraic spaces.

Corollary 3.1.12. Let $S$ be an algebraic space. If $S_{\text {red }}$ is a scheme then $S$ is a scheme.

Proof. Working Zariski-locally on $S_{\text {red }}$ is the same as working Zariski-locally on $S$, so we may arrange that $S_{\text {red }}$ is an affine scheme. Hence, $S_{\text {red }}$ is quasi-compact and separated, so $S$ is quasi-compact and separated. By Theorem 1.2.2, we may therefore write $S \simeq \lim S_{i}$ where $\left\{S_{i}\right\}$ is an inverse system of algebraic spaces of finite presentation over $\mathbf{Z}$. Since $\lim _{(}\left(S_{i}\right)_{\text {red }} \simeq \overleftarrow{S_{\text {red }}}$, this limit is an affine scheme. Thus, by Lemma A.3.3, there is an $i_{0}$ such that $\left(S_{i}\right)_{\text {red }}$ is a scheme for all $i \geq i_{0}$. But each $S_{i}$ is a noetherian algebraic space, so by [K. III, Thm. 3.3] it follows that $S_{i}$ is a scheme for all $i \geq i_{0}$. Hence, $S$ is a scheme since each map $S \rightarrow S_{i}$ is affine. 
As another application of Theorem 1.2.2, we can prove the following result via reduction to the finitely presented case over $\mathbf{Z}$ :

Theorem 3.1.13 (Deligne). Let $X \rightarrow S$ be a separated map of quasi-separated algebraic spaces equipped with a left $S$-action on $X$ by a finite group $G$. The quotient stack $[X / G]$ admits a coarse moduli space $\pi:[X / G] \rightarrow X / G$ such that the following hold:

(i) $X / G$ is separated over $S$.

(ii) The projection morphism $\pi_{X}: X \rightarrow X / G$ is affine and integral.

(iii) If $U \rightarrow X / G$ is a separated étale morphism, then the induced $G$-equivariant map

$$
U \times \times_{X / G} X \rightarrow U
$$

identifies $U$ with the quotient $\left(U \times_{X / G} X\right) / G$.

(iv) The pullback map

$$
\pi_{X}^{-1}:(\text { open subspaces of } X / G) \rightarrow(G \text {-invariant open subspaces of } X)
$$

is a bijection.

A stronger version, allowing non-constant $G$, is given in [Ry1, Thm. 5.4]. In our proof below, we rely on some results proved in the unpublished note [C3], but this seems permissible since Ry1 will be published and establishes a more general result.

Proof. By [C3, 1.1] the result holds when $X \rightarrow S$ is locally of finite presentation, and by the proof of [C3] 3.1] (using an argument from [SGA3, V, 4.1]) it also holds when $X$ is an affine scheme (for which it suffices to treat the case $S=\operatorname{Spec} \mathbf{Z})$. In fact, the proof shows that if $X=\operatorname{Spec}(A)$, then $X / G=\operatorname{Spec}\left(A^{G}\right)$; the verification of the universal property uses that we only consider maps to quasi-separated algebraic spaces in the definition of $X / G$ in 2.3 .

The general case can be deduced from these two cases as follows. By property (iii) and the existence of quotients by étale equivalence relations in the category of algebraic spaces, to prove the existence we may work étale locally on $S$. Hence, we may assume that $S$ is an affine scheme, so in particular $X$ is separated. Furthermore, by (iii) and (iv) we can use an elementary gluing argument for the Zariski topology (as at the start of [C2, §2]) to reduce to the case that $X$ is also quasi-compact. We apply absolute noetherian approximation in Theorem 1.2 .2 to the quasi-compact separated algebraic space $X$ (over Spec $\mathbf{Z}$ ) to get an isomorphism

$$
X \simeq \lim _{\lambda} X_{\lambda}
$$

where $\left\{X_{\lambda}\right\}$ is an inverse system of separated algebraic spaces of finite presentation (equivalently, finite type) over $\mathbf{Z}$ with affine transition maps. In particular, we can find an affine morphism

$$
X \rightarrow Y^{\prime}
$$

with $Y^{\prime}$ separated and finitely presented over $\mathbf{Z}$. Fix such a morphism.

The algebraic space $Y:=S \times_{\operatorname{Spec}} \mathbf{Z} Y^{\prime}$ is affine over $Y^{\prime}$, so we obtain an affine morphism

$$
\gamma: X \rightarrow Y
$$

over $S$ to an algebraic space that is separated and of finite presentation over $S$. Let $Z$ denote the $|G|$-fold fiber product of $Y$ with itself over $S$ :

$$
Z:=\prod_{g \in G} Y
$$

There is a left action of $G$ on $Z$ given by permuting the factors via $g_{0} \cdot\left(y_{g}\right)_{g \in G}=\left(y_{g g_{0}}\right)_{g \in G}$. Moreover, we have a $G$-equivariant map

$$
f: X \rightarrow Z, \quad x \mapsto(\gamma(g . x))_{g \in G} .
$$

This map $f$ is affine since it is equal to the composite

$$
X \stackrel{\Delta}{\longrightarrow} \prod_{g \in G} X \stackrel{\prod \gamma \circ g}{\longrightarrow} \prod_{g \in G} Y
$$


in which $\Delta$ is affine since $X$ is $S$-separated.

By the settled case when the structure map to $S$ is of finite presentation, the quotient $Z / G$ exists as an $S$-separated algebraic space and the projection map $Z \rightarrow Z / G$ is affine (by (ii)). We conclude that we can find an $S$-morphism

$$
\tau:[X / G] \rightarrow W
$$

from the stack-quotient $[X / G]$ to an $S$-separated algebraic space $W$ such that the composite map

$$
X \rightarrow[X / G] \rightarrow W
$$

is affine. We may replace $S$ with $W$, and then by working étale-locally on $S$ as before we are reduced to the settled case when $X$ is an affine scheme.

3.2. Finite type and finite presentation. In [C2, Thm. 4.3] it is proved that if $X \rightarrow S$ is a map of finite type between qcqs schemes then there is a closed immersion $i: X \hookrightarrow \bar{X}$ over $S$ into a finitely presented $S$-scheme $\bar{X}$, and that $\bar{X}$ can be taken to be separated over $S$ if $X$ is separated over $S$. This is the trick that, together with absolute noetherian approximation for qcqs schemes, allows one to reduce the proof of Nagata's theorem in the general scheme case to the case of schemes of finite type over $\mathbf{Z}$. We require an analogue for algebraic spaces, so we now aim to prove:

Theorem 3.2.1. Let $f: X \rightarrow S$ be a map of finite type between qcqs algebraic spaces. There exists a closed immersion $i: X \hookrightarrow \mathscr{X}$ over $S$ into an algebraic space $\mathscr{X}$ of finite presentation over $S$. If $X$ is $S$-separated then $\mathscr{X}$ may be taken to be $S$-separated.

To prove Theorem 3.2.1, we first need a gluing result for closed subspaces of algebraic spaces of finite presentation over a qcqs algebraic space $S$. Consider a commutative diagram of $S$-maps

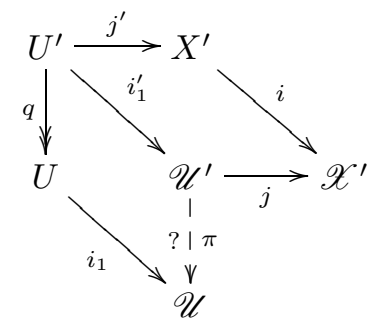

in which $q$ is a quasi-compact separated étale cover, $j$ is an open immersion, the maps $i_{1}, i_{1}^{\prime}$, and $i$ are closed immersions into algebraic spaces that are finitely presented and separated over $S$, and the top part is cartesian (so $j^{\prime}$ is an open immersion). We wish to study the possibility that there exists a suitable map $\pi$ as indicated in (3.2.1.1).

Lemma 3.2.2. With notation and hypotheses as in (3.2.1.1), let $X=U \coprod_{U^{\prime}} X^{\prime}$ be the pushout of the upper left triangle formed by $j^{\prime}$ and $q$. If there is a quasi-compact separated étale map $\pi$ as shown in (3.2.1.1) that makes the left part cartesian then the natural $S$-map from $X$ to the algebraic space pushout $\mathscr{X}=\mathscr{U} \coprod_{\mathscr{U}} \mathscr{X}^{\prime}$ of $j$ along $\pi$ is a closed immersion, and $\mathscr{X}$ is finitely presented over $S$.

Proof. Given $\pi$, form the cartesian pushout diagram in qcqs algebraic spaces over $S$

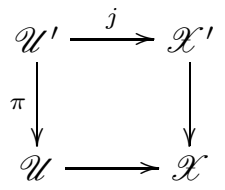

as in Lemma 3.1.6, so the bottom side is a quasi-compact open immersion (as $j$ is) and the right side is a quasi-compact separated étale surjection. In particular, $\mathscr{X}$ is finitely presented over $S$ since $\mathscr{X}^{\prime}$ is finitely presented over $S$. Consider the $S$-map of pushouts

$$
X=U \coprod_{U^{\prime}} X^{\prime} \rightarrow \mathscr{U} \coprod_{\mathscr{U}^{\prime}} \mathscr{X}^{\prime}=\mathscr{X} .
$$


It remains to prove that this is a closed immersion.

It is elementary to check that for $j \in\{1,2\}$, the diagram

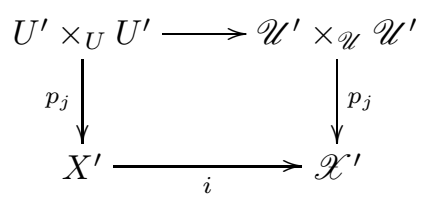

is cartesian, due to the cartesian property of both parallelogram sub-diagrams in (3.2.1.1). Since $X=X^{\prime} / R^{\prime}$ and $\mathscr{X}=\mathscr{X}^{\prime} / \mathscr{R}^{\prime}$ for

$$
R^{\prime}:=\left(U^{\prime} \times_{U} U^{\prime}\right) \coprod X^{\prime}, \quad \mathscr{R}^{\prime}:=\left(\mathscr{U}^{\prime} \times_{\mathscr{U}} \mathscr{U}^{\prime}\right) \coprod \mathscr{X}^{\prime},
$$

we may apply Lemma 3.1 .4 to infer that the commutative diagram

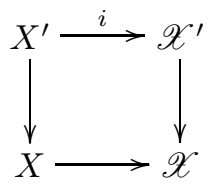

with étale surjective vertical maps is cartesian. Hence, the bottom side is a closed immersion since the top side is a closed immersion.

Given an arbitrary diagram of type (3.2.1.1) (with hypotheses on the maps as indicated there), the existence of $\pi$ as in Lemma 3.2.2 is quite subtle (and likely false). However, we can always modify $\mathscr{U}, \mathscr{U}^{\prime}$, and $\mathscr{X}^{\prime}$ so that the resulting diagram admits a $\pi$. More precisely, we have the following.

Proposition 3.2.3. Given a diagram (3.2.1.1), there is another one with the same $j^{\prime}$ and $q$ and for which an arrow $\pi$ as in Lemma 3.2.2 exists.

This says that, given a pushout diagram in the category of algebraic spaces of the type encountered in the upper left of (3.2.1.1) with $j^{\prime}$ and $q$, if the objects $U, U^{\prime}$, and $X^{\prime}$ individually admit closed immersions over $S$ into finitely presented algebraic spaces separated over $S$ (and satisfy a compatibility as expressed by the auxiliary map $j$ ) then we can choose such immersions so that an arrow $\pi$ as in Lemma 3.2 .2 exists.

Proof of Proposition 3.2.3. The proof proceeds in several steps.

Step 1 (replacing $\mathscr{U}$ ): Let us first show that there is a finitely presented closed subspace $\mathscr{V}$ in $\mathscr{U}$ which is better than $\mathscr{U}$ in the sense that it not only contains $U$ as a closed subspace but also admits a quasi-compact, étale, separated covering $h: \mathscr{V}^{\prime} \rightarrow \mathscr{V}$ whose pullback over $U$ is $q: U^{\prime} \rightarrow U$. The defect, which will have to be confronted in subsequent steps, is that $\mathscr{V}^{\prime}$ will have no evident connection with $\mathscr{U}^{\prime}$ (or even with $\mathscr{X}^{\prime}$ ) apart from containing $U^{\prime}$ as a closed subspace.

Lemma 3.2.4. There is a cartesian diagram of algebraic spaces over $S$

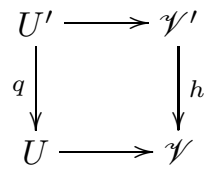

such that the bottom arrow is an inclusion of closed subspaces of $\mathscr{U}$ with $\mathscr{V}$ finitely presented over $S$, and $h$ is quasi-compact, étale, and separated.

If $U$ were finitely presented over $S$ then we could take $\mathscr{V}=U$ and $h=q$. The point of the lemma is to "spread out" $q$ over a finitely presented closed subspace of $\mathscr{U}$, whereas $U$ is merely finite type (and quasi-separated) over $S$ so its quasi-coherent ideal in $\mathscr{O}_{\mathscr{U}}$ may not be locally finitely generated. 
Proof. Consider the given closed immersion $i_{1}: U \hookrightarrow \mathscr{U}$ over $S$, with $\mathscr{U}$ a finitely presented algebraic space over $S$. Since $\mathscr{U}$ is a qcqs algebraic space, the quasi-coherent ideal $\mathscr{I} \subseteq \mathscr{O}_{\mathscr{U}}$ cutting out $U$ can be expressed as the direct limit $\lim \mathscr{I}_{\lambda}$ of its quasi-coherent subsheaves of finite type [RG, I, 5.7.8]. Hence, $U=\lim ^{\longleftarrow} \mathscr{U}_{\lambda}$ where $\mathscr{U}_{\lambda} \hookrightarrow \mathscr{U}$ is cut out by $\mathscr{I}_{\lambda}$.

The inverse system $\left\{\mathscr{U}_{\lambda}\right\}$ of finitely presented algebraic spaces over $S$ has affine transition maps and limit $U$, and every qcqs algebraic space (such as any $\mathscr{U}_{\lambda}$ ) is affine over an algebraic space of finite presentation over $\mathbf{Z}$ (by Theorem 1.2.2 which was proved in 3.1). Thus, by Proposition A.3.4 and Corollary A.3.5 we deduce that the quasi-compact étale separated cover $U^{\prime} \rightarrow U$ descends to a quasi-compact étale separated cover $\mathscr{V}^{\prime} \rightarrow \mathscr{U}_{\lambda_{0}}$ for some sufficiently large $\lambda_{0}$. Rename this $\mathscr{U}_{\lambda_{0}}$ as $\mathscr{V}$.

We may and do replace $\mathscr{U}$ in (3.2.1.1) with $\mathscr{V}$, but we emphasize again that $\mathscr{V}^{\prime}$ is unrelated to $\mathscr{U}^{\prime}$ (except that both contain $U^{\prime}$ as a closed subspace).

Returning to the task of constructing $\pi$ as in (3.2.1.1) after a suitable change in $\mathscr{U}^{\prime}$ (and leaving $j^{\prime}$ and $q$ unchanged), the strategy is to make an initial change in $\mathscr{U}^{\prime}$ (as a quasi-compact open subspace of $\mathscr{X}^{\prime}$ meeting $X^{\prime}$ in $U^{\prime}$ ) and to then show that by replacing $\mathscr{X}^{\prime}$ and $\mathscr{U}=\mathscr{V}$ with suitable finitely presented closed subspaces (respectively containing $X^{\prime}$ and $U$ ) and replacing the $\mathscr{U}$-spaces $\mathscr{U}^{\prime}$ and $\mathscr{V}^{\prime}$ with the respective pullback closed subspaces containing $U^{\prime}$ we eventually get to a situation in which we can identify $\mathscr{V}^{\prime}$ and $\mathscr{U}^{\prime}$ over $S$ in a manner that respects how $U^{\prime}$ is a closed subspace of each. In such a favorable situation the map $h: \mathscr{V}^{\prime} \rightarrow \mathscr{U}$ then serves as the desired map $\pi$.

To carry this out, we have to overcome the essential difficulty in comparison with the Zariski-gluing problem faced in the scheme case as considered in [C2, Thm. 4.3]: whereas $U^{\prime}$ is open in $X^{\prime}$, it is only étale (rather than Zariski-open) over $U$, and so rather than trying to spread $U^{\prime}$ to a common open subspace of $\mathscr{X}^{\prime}$ and $\mathscr{U}$ (after suitable shrinking on these two spaces) we are instead trying to spread $U^{\prime}$ to an open subspace of $\mathscr{X}^{\prime}$ that is quasi-compact, étale, and separated over $\mathscr{U}$.

Let $\left\{\mathscr{X}_{\alpha}^{\prime}\right\}$ denote the inverse system consisting of all finitely presented closed subspaces in $\mathscr{X}^{\prime}$ containing $X^{\prime}$ and let $\left\{\mathscr{U}_{\beta}\right\}$ denote the inverse system of finitely presented closed subspaces in $\mathscr{U}$ containing $U$, so

$$
X^{\prime}=\lim _{\longleftarrow} \mathscr{X}_{\alpha}^{\prime}, \quad U=\lim _{\longleftarrow} \mathscr{U}_{\beta} .
$$

Consider the diagram of cartesian squares

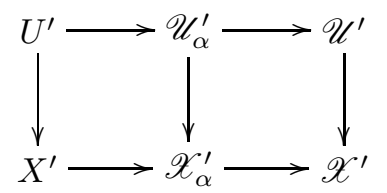

over $S$ in which the horizontal maps are closed immersions and the vertical maps are quasi-compact open immersions. Passing to the limit gives $U^{\prime} \simeq \lim _{\longleftarrow} \mathscr{U}_{\alpha}^{\prime}$ over $S$, with $\left\{\mathscr{U}_{\alpha}^{\prime}\right\}$ an inverse system of finitely presented closed subspaces of $\mathscr{U}^{\prime}$ containing $U^{\prime}$.

Likewise, consider the diagram of cartesian squares

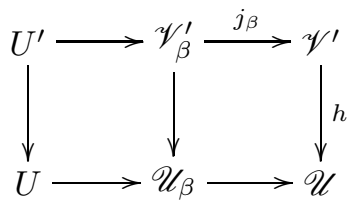

over $S$ in which the horizontal maps are closed immersions and the vertical maps are quasi-compact, étale, and separated. Passing to the limit gives another limit presentation of $U^{\prime}$ over $S$, namely $U^{\prime}=\lim _{\mathscr{\beta}} \mathscr{V}_{\beta}^{\prime}$. The situation we would like to reach is that for some large $\alpha_{0}$ and $\beta_{0}$ there is an $S$-isomorphism $\mathscr{V}_{\beta_{0}}^{\prime} \simeq \mathscr{U}_{\alpha_{0}}^{\prime}$ respecting the closed immersions of $U^{\prime}$ into each side, as we could then rename $\mathscr{V}_{\beta_{0}}^{\prime}$ as $\mathscr{V}^{\prime}$ and $\mathscr{U}_{\alpha_{0}}^{\prime}$ as $\mathscr{U}^{\prime}$ to get to the case $\mathscr{V}^{\prime}=\mathscr{U}^{\prime}$ that provides $\pi$ as desired. We will not find such a pair $\left(\alpha_{0}, \beta_{0}\right)$ after making changes in various auxiliary spaces, but we will do something close to this which is sufficient for the purpose of proving Proposition 3.2 .3 . 
Step 2 (shrinking $\mathscr{U}^{\prime}$ and $\mathscr{V}^{\prime}$ so that they become related): A key observation is that we may replace $\mathscr{U}^{\prime}$ with any finitely presented closed subspace containing $U^{\prime}$. To justify this, we have to prove that any finitely presented closed subspace $\mathscr{U}_{1}^{\prime}$ in $\mathscr{U}^{\prime}$ containing $U^{\prime}$ extends to a finitely presented closed subspace $\mathscr{X}_{1}^{\prime}$ in $\mathscr{X}^{\prime}$ containing $X^{\prime}$. (Recall that $\mathscr{U}^{\prime}$ is open in $\mathscr{X}^{\prime}$ and $X^{\prime}$ is closed in $\mathscr{X}^{\prime}$, with $\mathscr{U}^{\prime} \cap X^{\prime}=$ $\mathscr{U}^{\prime} \times \mathscr{X}^{\prime} X^{\prime}=U^{\prime}$.) This is a standard extension argument with quasi-coherent sheaves, except that we are working with algebraic spaces rather than with schemes, and it goes as follows. Let $\mathscr{I}_{X^{\prime}} \subseteq \mathscr{O}_{\mathscr{X}^{\prime}}$ denote the quasi-coherent ideal that cuts out $X^{\prime}$ inside of $\mathscr{X}^{\prime}$. The finitely presented closed subspace $\mathscr{U}_{1}^{\prime}$ in the quasi-compact open subspace $\mathscr{U}^{\prime} \subseteq \mathscr{X}^{\prime}$ is cut out by a finite type quasi-coherent ideal sheaf $\mathscr{I} \subseteq \mathscr{O}_{\mathscr{U}^{\prime}}$, and since $\mathscr{U}^{\prime} \cap X^{\prime}=U^{\prime} \subseteq \mathscr{U}_{1}^{\prime}$ we have $\left.\mathscr{I} \subseteq \mathscr{I}_{X^{\prime}}\right|_{\mathscr{U}^{\prime}}$. Let $\mathscr{K} \subseteq \mathscr{O}_{\mathscr{X}}$, be the quasi-coherent ideal corresponding to the schematic closure of $\mathscr{U}_{1}^{\prime}$ inside of $\mathscr{X}^{\prime}$. (Explicitly, if $j: \mathscr{U}^{\prime} \hookrightarrow \mathscr{X}^{\prime}$ denotes the quasi-compact open immersion then $\mathscr{K}$ is the preimage of $j_{*}(\mathscr{I})$ under $\mathscr{O}_{\mathscr{X}^{\prime}} \rightarrow j_{*}\left(\mathscr{O}_{\mathscr{U}^{\prime}}\right)$.) This is a quasi-coherent ideal sheaf whose intersection with $\mathscr{I}_{X^{\prime}}$ inside of $\mathscr{O}_{\mathscr{X}}$, restricts to the finite type $\mathscr{I}$ on the quasi-compact open $\mathscr{U}^{\prime}$. On the qcqs algebraic space $\mathscr{X}^{\prime}$ we can express $\mathscr{K}$ as the direct limit of its finite type quasi-coherent subsheaves RG, I, 5.7.8], so the finite type quasi-coherent ideal $\mathscr{I}$ on $\mathscr{U}^{\prime}$ extends to to a finite type quasi-coherent ideal $\mathscr{J} \subseteq \mathscr{I}_{X^{\prime}}$. The finitely presented closed subspace $\mathscr{X}_{1}^{\prime} \hookrightarrow \mathscr{X}^{\prime}$ cut out by $\mathscr{J}$ fits into a commutative diagram

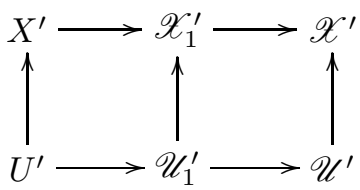

in which the vertical maps are open immersions, the horizontal maps are closed immersions, the right square is cartesian by definition of $\mathscr{X}_{1}^{\prime}$, and the outside edge is cartesian since $X^{\prime} \cap \mathscr{U}^{\prime}=U^{\prime}$ as quasi-compact subspaces of $\mathscr{X}^{\prime}$. Hence, the left square in (3.2.4.1) is cartesian, so $\mathscr{U}_{1}^{\prime}$ and $\mathscr{X}_{1}$ are as required.

To make a useful initial shrinking of $\mathscr{U}^{\prime}$ as just considered, we shall use $\mathscr{V}^{\prime}$ as a guide. Since $\mathscr{V}^{\prime}$ is finitely presented over $S$, by Proposition A.3.1 we have $\operatorname{Hom}_{S}\left(U^{\prime}, \mathscr{V}^{\prime}\right)=\lim _{\longrightarrow} \operatorname{Hom}_{S}\left(\mathscr{U}_{\alpha}^{\prime}, \mathscr{V}^{\prime}\right)$. Applying this to the closed immersion $\lim _{\longleftarrow} \mathscr{U}_{\alpha}^{\prime}=U^{\prime} \hookrightarrow \mathscr{V}^{\prime}$ over $S$ as in Lemma 3.2 .4 gives an $\alpha_{0}$ for which there is a commutative diagram of $S$-maps

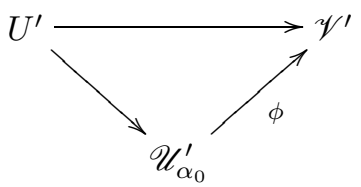

in which the left diagonal and top arrows are the canonical closed immersions. Thus, upon renaming such a $\mathscr{U}_{\alpha_{0}}^{\prime}$ as $\mathscr{U}^{\prime}$ (as we may), we get a commutative diagram of $S$-maps

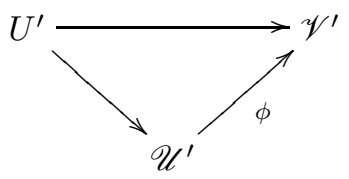

in which the unlabeled maps are the canonical closed immersions. Likewise, since the new $\mathscr{U}^{\prime}$ is finitely presented over $S$ we have

$$
\operatorname{Hom}_{S}\left(U^{\prime}, \mathscr{U}^{\prime}\right)=\lim _{\longrightarrow} \operatorname{Hom}_{S}\left(\mathscr{V}_{\beta}^{\prime}, \mathscr{U}^{\prime}\right)
$$

so there is a $\beta_{0}$ for which we may construct a commutative diagram

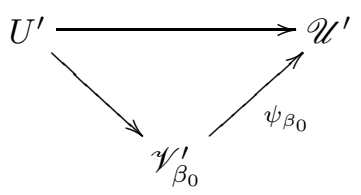

over $S$ in which the unlabeled maps are the canonical closed immersions. 
For $\beta \geq \beta_{0}$, define $\psi_{\beta}: \mathscr{V}_{\beta}^{\prime} \rightarrow \mathscr{U}^{\prime}$ to be the composition of $\psi_{\beta_{0}}$ with the closed immersion $\mathscr{V}_{\beta}^{\prime} \hookrightarrow \mathscr{V}_{\beta_{0}}^{\prime}$. We concatenate (3.2.4.3) and the variant of (3.2.4.4) for $\beta \geq \beta_{0}$ to get the commutative diagram

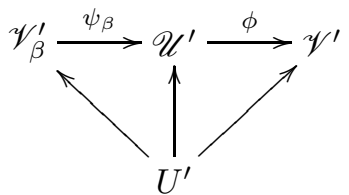

over $S$ in which the maps from $U^{\prime}$ are the canonical closed immersions. Since the family of closed immersions $U^{\prime} \rightarrow \mathscr{V}_{\beta}^{\prime}$ over $S$ becomes the $S$-isomorphism $U^{\prime} \simeq \lim _{\beta} \mathscr{V}_{\beta}^{\prime}$ upon passing to the limit, the family of $S$-maps $\phi \circ \psi_{\beta}: \mathscr{V}_{\beta}^{\prime} \rightarrow \mathscr{V}^{\prime}$ and the family of $S$-maps $j_{\beta}: \mathscr{V}_{\beta}^{\prime} \hookrightarrow \mathscr{V}^{\prime}$ given by the canonical closed immersions have limit maps $U^{\prime} \rightrightarrows \mathscr{V}^{\prime}$ which coincide (with the canonical closed immersion). But $U^{\prime}=\lim _{\longleftarrow} V_{\beta}^{\prime}$, so Proposition A.3.1 gives

$$
\operatorname{Hom}_{S}\left(U^{\prime}, \mathscr{V}^{\prime}\right)=\lim _{\longrightarrow} \operatorname{Hom}_{S}\left(\mathscr{V}_{\beta}^{\prime}, \mathscr{V}^{\prime}\right)
$$

Thus, for some $\beta_{1} \geq \beta_{0}$ the $S$-map $\phi \circ \psi_{\beta_{1}}: \mathscr{V}_{\beta_{1}}^{\prime} \rightarrow \mathscr{V}^{\prime}$ is equal to the canonical closed immersion $j_{\beta_{1}}$.

Now consider the diagram of $S$-maps (with $\psi:=\psi_{\beta_{1}}$ )

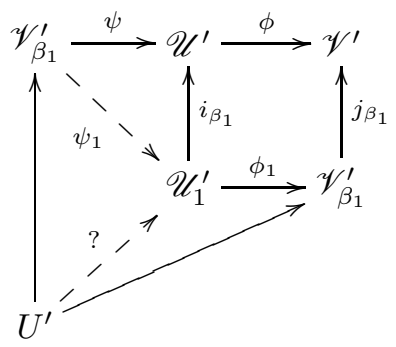

in which the new algebraic space $\mathscr{U}_{1}^{\prime}$ and the maps $i_{\beta_{1}}$ and $\phi_{1}$ from it to $\mathscr{U}^{\prime}$ and $\mathscr{V}_{\beta_{1}}^{\prime}$ are defined to make the right square be cartesian (so $\mathscr{U}_{1}^{\prime}$ is a finitely presented closed subspace of $\mathscr{U}^{\prime}$ ) and the composite map $\phi \circ \psi$ across the top equals the map $j_{\beta_{1}}$ along the right side. Thus, there is a unique $\psi_{1}$ making the upper triangle commute (uniqueness because $i_{\beta_{1}}$ is monic), and the composite $\phi_{1} \circ \psi_{1}$ is the identity map either by construction or because

$$
j_{\beta_{1}} \circ\left(\phi_{1} \circ \psi_{1}\right)=\phi \circ i_{\beta_{1}} \circ \psi_{1}=\phi \circ \psi=j_{\beta_{1}}
$$

with $j_{\beta_{1}}$ monic. The canonical closed immersion from $U^{\prime}$ to $\mathscr{V}_{\beta_{1}}^{\prime}$ is used along the left and bottom sides of the diagram, and we define the dotted arrow from $U^{\prime}$ to $\mathscr{U}_{1}^{\prime}$ by composing the left side with $\psi_{1}$. In particular, $\mathscr{U}_{1}^{\prime}$ as a (finitely presented) closed subspace of $\mathscr{U}^{\prime}$ contains $U^{\prime}$. Since $\phi_{1} \circ \psi_{1}=\mathrm{id}_{\mathscr{H}_{\beta_{1}}^{\prime}}$, the bottom triangle also commutes. Thus, we have filled in all of the dotted arrows to make (3.2.4.5) a commutative diagram over $S$.

Using the argument at the beginning of Step 2, we can extend $\mathscr{U}_{1}^{\prime}$ to a finitely presented closed subspace $\mathscr{X}_{1}^{\prime}$ in $\mathscr{X}^{\prime}$ containing $X^{\prime}$. We may replace $\mathscr{U}^{\prime} \hookrightarrow \mathscr{X}^{\prime}$ with $\mathscr{U}_{1}^{\prime} \hookrightarrow \mathscr{X}_{1}^{\prime}, \mathscr{V}=\mathscr{U}$ with $\mathscr{U}_{\beta_{1}}$, $\mathscr{V}^{\prime}$ with $\mathscr{V}_{\beta_{1}}^{\prime}=\mathscr{V}^{\prime} \times_{\mathscr{U}} \mathscr{U}_{\beta_{1}}$, and the maps $\phi$ and $\psi$ with $\phi_{1}$ (renamed as $s$ ) and $\psi_{1}$ (renamed as $t$ ) respectively to arrive at the case when the conclusion of Lemma 3.2.4 still holds with $\mathscr{V}=\mathscr{U}$ but there is also a commutative diagram

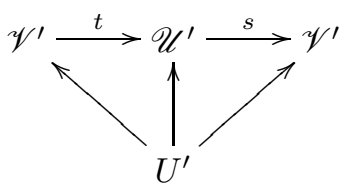

whose composite across the top is the identity map (and whose non-horizontal arrows are the canonical closed immersions). If $t \circ s=\mathrm{id}_{\mathscr{U}^{\prime}}$ then $s$ and $t$ would be inverse isomorphisms respecting the closed immersions from $U^{\prime}$, so we would be done (taking $\pi$ to be $\mathscr{U}^{\prime} \simeq \mathscr{V}^{\prime} \stackrel{h}{\rightarrow} \mathscr{V}=\mathscr{U}$ ). It remains to handle the possibility $t \circ s \neq \operatorname{id} \mathscr{U}^{\prime}$. 
Step 3 (making inverse maps): We shall construct suitable further shrinkings of $\mathscr{U}^{\prime}$ and $\mathscr{V}^{\prime}$ compatibly with $s$ and $t$ simultaneously so that we can get to the case where $t \circ s=\mathrm{id}_{\mathscr{U}}$. The commutativity of (3.2.4.6) implies that the composite $S$-map

$$
U^{\prime} \hookrightarrow \mathscr{U}^{\prime} \stackrel{s}{\rightarrow} \mathscr{V}^{\prime} \stackrel{t}{\rightarrow} \mathscr{U}^{\prime}
$$

is the canonical closed immersion. But $U^{\prime}=\lim \mathscr{U}_{\alpha}^{\prime}$ and the target $\mathscr{U}^{\prime}$ is finitely presented over $S$, so Proposition A.3.1 provides an $\alpha_{2}$ such that the composite $S$-map

$$
\mathscr{U}_{\alpha_{2}}^{\prime} \hookrightarrow \mathscr{U}^{\prime} \stackrel{s}{\rightarrow} \mathscr{V}^{\prime} \stackrel{t}{\rightarrow} \mathscr{U}^{\prime}
$$

is the canonical closed immersion. Let $\mathscr{U}_{2}^{\prime}=\mathscr{U}_{\alpha_{2}}^{\prime}$ and $\mathscr{V}_{2}^{\prime}=t^{-1}\left(\mathscr{U}_{\alpha_{2}}^{\prime}\right)$, so it makes sense to restrict $s$ and $t$ to get $S$-maps

$$
\mathscr{U}_{2}^{\prime} \stackrel{s_{2}}{\rightarrow} \mathscr{V}_{2}^{\prime} \stackrel{t_{2}}{\rightarrow} \mathscr{U}_{2}^{\prime}
$$

whose composition is the identity map (as may be checked upon composing on the right with $\mathscr{U}_{2}^{\prime} \hookrightarrow \mathscr{U}^{\prime}$ ). Moreover, since $s \circ t=\mathrm{id}_{\mathscr{U}}{ }^{\prime}$ we have that $s_{2} \circ t_{2}=\mathrm{id}_{\mathscr{V}_{2}^{\prime}}$. Hence, $s_{2}$ and $t_{2}$ are inverse $S$-isomorphisms! But beware that we are not done, since the commutative square

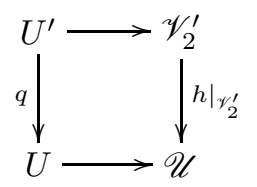

may fail to be cartesian, and more seriously the map $\left.h\right|_{\mathscr{V}_{2}^{\prime}}$ may not be étale (though it is certainly quasicompact and separated). Hence, we cannot simply rename $\mathscr{V}_{2}^{\prime}$ as $\mathscr{V}^{\prime}$ without destroying the properties that make $\mathscr{V}^{\prime}$ useful in the first place, as in Lemma 3.2.4

We can fix this difficulty as follows. Since $U=\lim \mathscr{U}_{\alpha}$ for the inverse system $\left\{\mathscr{U}_{\alpha}\right\}$ of finitely presented closed subspaces of $\mathscr{U}$, the cartesian property in Lemma 3.2.4 implies that $U^{\prime}=\lim h^{-1}\left(\mathscr{U}_{\alpha}\right)$. Hence, there exists $\alpha_{3} \geq \alpha_{2}$ such that the finitely presented closed subspace $\mathscr{V}_{3}^{\prime}:=h^{-1}\left(\mathscr{U}_{\alpha_{3}}\right)$ in $\mathscr{V}^{\prime}$ is contained in $\mathscr{V}_{2}^{\prime}$ because $\mathscr{V}_{2}^{\prime}$ is finitely presented inside of $\mathscr{V}^{\prime}$. Obviously the commutative diagram

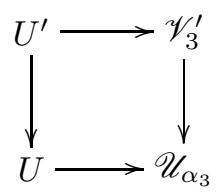

is cartesian and it is étale along the right side. Thus, if we let $\mathscr{U}_{3}^{\prime} \subseteq \mathscr{U}_{2}^{\prime}$ correspond to $\mathscr{V}_{3}^{\prime} \subseteq \mathscr{V}_{2}^{\prime}$ via the inverse $S$-isomorphisms $s_{2}$ and $t_{2}$ then we can replace $\mathscr{U}$ with $\mathscr{U}_{\alpha_{3}}$ and use $\mathscr{U}_{3}^{\prime}$ in the role of $\mathscr{U}_{1}^{\prime}$ back in the construction of $\mathscr{X}_{1}^{\prime}$ in (3.2.4.1) to get a finitely presented closed subspace $\mathscr{X}_{3}^{\prime} \subseteq \mathscr{X}^{\prime}$ for which the preceding cartesian difficulty does not arise. Hence, the $S$-isomorphism $\mathscr{U}_{3}^{\prime} \simeq \mathscr{V}_{3}^{\prime}$ via $s_{2}$ and $t_{2}$ has the required properties to provide the equality $\mathscr{U}^{\prime}=\mathscr{V}^{\prime}$ that we sought to construct.

We are now in a position to prove Theorem 3.2.1

Proof of Theorem 3.2.1. We again proceed in three steps.

Step 1 (separatedness): Let us first check that once the existence of $i$ is proved then we can find such an $\mathscr{X}$ that is $S$-separated if $X$ is $S$-separated. Make an initial choice of $\mathscr{X}$, and let $\mathscr{I}$ denote the quasi-coherent ideal of $X$ in $\mathscr{X}$. By [RG, I, 5.7.8], $\mathscr{I}=\lim _{\mathscr{I}_{\alpha}}$ for the directed system of finite type quasi-coherent ideal sheaves $\mathscr{I}_{\alpha}$ in $\mathscr{I}$. Let $\mathscr{X}_{\alpha} \subseteq \mathscr{X}$ be the closed subspace cut out by $\mathscr{I}_{\alpha}$, so each $\mathscr{X}_{\alpha}$ is finitely presented over $S$ and $\lim _{\leftarrow} \mathscr{X}_{\alpha}=X$ is $S$-separated. It suffices to prove that $\mathscr{X}_{\alpha}$ is $S$-separated for sufficiently large $\alpha$. We may work étale-locally on $S$, so we may assume $S$ is affine. 
Since $\mathscr{X} \rightarrow S$ is finitely presented, by absolute noetherian approximation for affine schemes (such as $S$ ) and Proposition A.3.4 there is a cartesian diagram of algebraic spaces

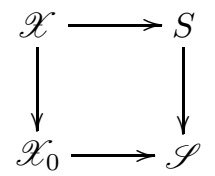

with $\mathscr{S}$ affine of finite type over $\mathbf{Z}$ and the bottom side of finite presentation. The normalization of $\mathscr{X}_{0, \text { red }}$ is therefore a finite surjective map of algebraic spaces $\mathscr{X}_{1} \rightarrow \mathscr{X}_{0}$ (so $\mathscr{X}_{1}$ is finitely presented over $\mathbf{Z}$ ), and by Proposition 2.3.1 (which rests on A.1$_{\text {A.2. }}$ and there is a finite surjective map $\mathscr{X}_{1}^{\prime} \rightarrow \mathscr{X}_{1}$ with $\mathscr{X}_{1}^{\prime}$ a scheme. This finite map is finitely presented, since its target is finitely presented over $\mathbf{Z}$. Applying the affine base change by $S \rightarrow \mathscr{S}$, we get a finite and finitely presented surjective map $\pi: \mathscr{X}^{\prime} \rightarrow \mathscr{X}$ from a scheme.

Define $\mathscr{X}_{\alpha}^{\prime}=\pi^{-1}\left(\mathscr{X}_{\alpha}\right)$, so $\lim \mathscr{X}_{\alpha}^{\prime}=\pi^{-1}(X)$ is separated. Each $\mathscr{X}_{\alpha}^{\prime}$ is finitely presented over $S$ since $\mathscr{X}_{\alpha}$ is finitely presented over $S$ and $\pi$ is finitely presented. Since $\left\{\mathscr{X}_{\alpha}^{\prime}\right\}$ is an inverse system of finitely presented $S$-schemes with affine transition maps, by applying [TT, Thm. C.7] over the affine $S$ it follows that $\mathscr{X}_{\alpha}^{\prime}$ is separated for sufficiently large $\alpha$. Finiteness and surjectivity of $\pi$ then gives that the algebraic space $\mathscr{X}_{\alpha}$ of finite presentation over the affine $S$ is separated for sufficiently large $\alpha$, as desired.

We may now ignore separatedness considerations and focus on merely constructing a closed immersion $i: X \hookrightarrow \mathscr{X}$ over $S$ into an algebraic space $\mathscr{X}$ of finite presentation over $S$.

Step 2 (proof when $S$ is a scheme, by induction on $X$-stratification):

Assume $S$ is a scheme. Applying Theorem 3.1.1 to the qcqs algebraic space $X$ yields a rising union of quasi-compact open subspaces

$$
\emptyset=X_{0} \subseteq X_{1} \subseteq \cdots \subseteq X_{s-1} \subseteq X_{s}=X
$$

whose (locally closed) strata are schemes (with their reduced structure). Let us now proceed by induction on $s$, the case $s=0$ being trivial. Thus, we may assume $s \geq 1$ and that the result is known for $U=X_{s-1}$ (a quasi-compact open subspace of $X$ ).

Let $X^{\prime} \rightarrow X$ be an étale covering by an affine scheme (so $X^{\prime} \rightarrow X$ is quasi-compact and separated as well, hence finitely presented). The pullback $U^{\prime}=U \times_{X} X^{\prime}$ is a quasi-compact open subspace of the scheme $X^{\prime}$ (so it is a scheme) and the projection $q: U^{\prime} \rightarrow U$ is finitely presented, separated, and étale. The inductive hypothesis provides a closed immersion $U \hookrightarrow \mathscr{U}$ into a finitely presented algebraic space over the scheme $S$. Moreover, since $X^{\prime} \rightarrow S$ is a finite type map of schemes, by the known scheme case [C2, Thm. 4.3] there is a closed immersion $i: X^{\prime} \hookrightarrow \mathscr{X}^{\prime}$ into a scheme $\mathscr{X}^{\prime}$ of finite presentation over $S$. The quasi-compact open subscheme $U^{\prime} \subseteq X^{\prime}$ has the form $X^{\prime} \cap \mathscr{U}^{\prime}$ for a quasi-compact open subscheme $\mathscr{U}^{\prime} \subseteq \mathscr{X}^{\prime}$. Applying Proposition 3.2 .3 to the resulting diagram of type (3.2.1.1), we can use Lemma 3.2 .2 to find a closed immersion $X \hookrightarrow \mathscr{X}$ over $S$ with $\mathscr{X}$ of finite presentation over $S$, as desired.

Step 3 (proof for general $S$, by induction on $S$-stratification): Consider a rising union

$$
\emptyset=U_{0} \subseteq U_{1} \subseteq \cdots \subseteq U_{r}=S
$$

of quasi-compact open subspaces as in Theorem 3.1.1. Let $X_{i}=X \times_{S} U_{i}$. We shall proceed by induction on $r$. The case $r=0$ is trivial, so we can assume $r \geq 1$ and that $U:=X_{r-1}$ has a closed immersion over $U_{r-1}$ into a finitely presented algebraic space $\mathscr{U}$ over $U_{r-1}$ (which in turn is finitely presented over $S$ since $U_{r-1}$ is a quasi-compact open subspace of the qcqs $S$ ).

Let $\varphi: Y \rightarrow U_{r}=S$ be an étale covering by a quasi-compact separated (e.g., affine) scheme, so $\varphi$ is a quasi-compact separated étale map (hence of finite presentation) and $X^{\prime}:=X \times_{S} Y$ is an algebraic space that is separated and of finite type over $Y$. Since $U_{r-1}$ is a quasi-compact open subspace of $S$, the algebraic space $X^{\prime}$ contains $U^{\prime}=X^{\prime} \times_{S} U_{r-1}$ as a quasi-compact open subspace such that the natural map $q: U^{\prime} \rightarrow X \times{ }_{S} U_{r-1}=X_{r-1}=: U$ is quasi-compact, étale, and separated. But we noted that by induction on $r$ there is a closed immersion $U \hookrightarrow \mathscr{U}$ over $U_{r-1}$ into a finitely presented algebraic space over $U_{r-1}$, and that $\mathscr{U}$ is also finitely presented over $S$. 
By the settled case when the base is a scheme (Step 2), the separated finite type morphism $X^{\prime} \rightarrow Y$ to the qcqs scheme $Y$ admits a closed immersion $i: X^{\prime} \hookrightarrow \mathscr{X}^{\prime}$ over $Y$ into an algebraic space of finite presentation over $Y$. By composing $\mathscr{X}^{\prime} \rightarrow Y$ with the finitely presented $\varphi: Y \rightarrow S, i$ may also be viewed as a closed immersion over $S$ into an algebraic space that is separated and of finite presentation over $S$. Since $X^{\prime}$ is a closed subspace of $\mathscr{X}^{\prime}$ (via $i$ ), the quasi-compact open subspace $U^{\prime} \subseteq X^{\prime}$ has the form $X^{\prime} \cap \mathscr{U}^{\prime}$ for a quasi-compact open subspace $\mathscr{U}^{\prime} \subseteq \mathscr{X}^{\prime}$.

These spaces fit together into a diagram of the form (3.2.1.1), but possibly without a map $\pi: \mathscr{U}^{\prime} \rightarrow \mathscr{U}$ as in Lemma 3.2.2 But applying Proposition 3.2 .3 puts us in the case when the map $\pi$ does exist, so we may then apply Lemma 3.2 .2 to complete the proof of Theorem 3.2.1.

To conclude this section, let us use Theorem 3.2.1 to prove Theorem 1.2.1 in general; this includes the general noetherian case, which has not yet been proved without excellence hypotheses. Note that in $\$ 2$ we already settled the case when $S$ is of finite presentation over $\mathbf{Z}$. By Theorem 3.2.1 there is a closed immersion $i: X \hookrightarrow X^{\prime}$ (over $S$ ) into an algebraic space $X^{\prime}$ that is separated and of finite presentation over $S$. If $X^{\prime}$ admits an $S$-compactification $j: X^{\prime} \hookrightarrow \bar{X}^{\prime}$ then the composite immersion $j \circ i: X \rightarrow \bar{X}^{\prime}$ over $S$ is quasi-compact, so its scheme-theoretic image $\bar{X} \subseteq \bar{X}^{\prime}$ is an $S$-compactification of $X$. Hence, it suffices to treat $X^{\prime}$ instead of $X$, so we may and do now assume that $X$ is finitely presented (and separated) over $S$.

By Theorem 1.2.2 (which was proved in 93.1 ) there is an isomorphism $S \simeq \lim S_{\alpha}$ with $\left\{S_{\alpha}\right\}$ an inverse system with affine transition maps between algebraic spaces of finite presentation over $\mathbf{Z}$. Choose an étale cover $U \rightarrow X$ with $U$ an affine scheme, so $U$ and $R=U \times_{X} U$ are schemes of finite presentation over $S$. By Proposition A.3.4 for sufficiently large $\alpha_{0}$ there are schemes $R_{\alpha_{0}}$ and $U_{\alpha_{0}}$ of finite presentation over $S_{\alpha_{0}}$ such that for $U_{\alpha}:=S_{\alpha} \times_{S_{\alpha_{0}}} U_{\alpha_{0}}$ and $R_{\alpha}:=S_{\alpha} \times_{S_{\alpha_{0}}} R_{\alpha_{0}}$ we have $U=\lim U_{\alpha}$ and $R=\lim R_{\alpha}$.

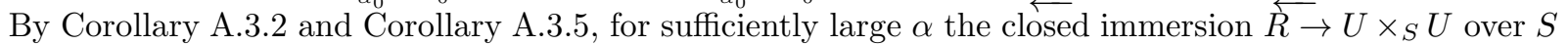
arises by base change from a closed immersion $R_{\alpha} \rightarrow U_{\alpha} \times_{S_{\alpha}} U_{\alpha}$ over $S_{\alpha}$ such that the projections $R_{\alpha} \rightrightarrows U_{\alpha}$ are étale. A further application of Corollary A.3.2 gives that $R_{\alpha}$ is thereby an equivalence relation on $U_{\alpha}$ for sufficiently large $\alpha$. Thus, $X_{\alpha}:=U_{\alpha} / R_{\alpha}$ is a separated algebraic space of finite presentation over $S_{\alpha}$ and it induces $X \rightarrow S$ after base change along $S \rightarrow S_{\alpha}$. Theorem 1.2.1 is already known for $X_{\alpha} \rightarrow S_{\alpha}$ since $S_{\alpha}$ is of finite presentation over $\mathbf{Z}$, so the theorem is now fully proved in general.

\section{Appendix A. Some foundational facts for algebraic spaces}

In $\$ 1.3$ we explained why the avoidance of quasi-separatedness hypotheses in the foundations of the theory of algebraic spaces is convenient when carrying out some of the gluing constructions that we need in the proof of Nagata's theorem for qcqs algebraic spaces. We also need to use the formalism of inverse limits (with affine transition maps) when the base object $S=\lim S_{\alpha}$ is an inverse limit of algebraic spaces $S_{\alpha}$ rather than of schemes, and so we have to prove certain results in this generality that are analogues of known results in the case of limits of schemes. It is convenient to not have to keep track of quasi-separatedness a priori in such limit constructions as well.

This appendix collects the results that we need from the theory of algebraic spaces and for which we do not know of a reference in the literature. We also provide some instructive examples of the surprises that can occur in the absence of quasi-separatedness (but the reader need not get worried about such things; for our purposes, the reason to avoid quasi-separatedness in the foundations is so that we can make sense of certain constructions prior to checking that the construction is quasi-separated in cases that we require).

A.1. Algebraic spaces without quasi-separatedness. In [RG, I, 5.7.2] it is proved that if $R \rightrightarrows U$ is an étale equivalence relation in schemes and $X=U / R$ is the quotient sheaf on the étale site of the category of schemes then the quotient map of sheaves $U \rightarrow X$ is representable by étale surjections of schemes. That is, for any map $W \rightarrow X$ from a scheme $W, U \times_{X} W$ is a scheme. Note that in this fiber product, the map $U \rightarrow X$ is rather special: it arises from an étale presentation of $X$.

To make the theory of algebraic spaces workable without quasi-separatedness hypotheses (with algebraic spaces defined to be étale sheaves admitting such a quotient presentation $U / R$ ), the essential step is to prove that if $V$ is any scheme then any map $V \rightarrow X$ is representable; that is, $V \times_{X} W$ is a scheme for any pair 
of maps $V \rightarrow X$ and $W \rightarrow X$ from schemes $V$ and $W$. Since $V \times_{X} W=(V \times W) \times_{X \times X} X$ (with absolute products over Spec $\mathbf{Z}$ ), this representability is a consequence of the following result.

Proposition A.1.1. For $X$ as above, the diagonal map $\Delta_{X}: X \rightarrow X \times_{\operatorname{Spec}} \mathbf{z} X$ is representable. More generally, if $X \rightarrow S$ is any map of algebraic spaces then $\Delta_{X / S}: X \rightarrow X \times_{S} X$ is representable.

Recall that a map $X \rightarrow Y$ of contravariant functors on the category of schemes is representable if $X \times_{Y} T$ is a scheme for any scheme $T$.

Proof. If $V$ is a scheme and $h: V \rightarrow X \times_{S} X$ is a map corresponding to a pair of maps $h_{1}, h_{2}: V \rightrightarrows X$ then $h$ is the composition $V \rightarrow V \times_{S} V \rightarrow X \times_{S} X$, where the second step is $h_{1} \times h_{2}$. Provided that $\Delta_{S}$ is representable, so $V \times_{S} V$ is a scheme, the representability of $\Delta_{X / S}^{-1}(V)$ reduces to the representability of $\Delta_{X / S}^{-1}\left(V \times_{S} V\right)=\Delta_{X}^{-1}\left(V \times_{S p e c} \mathbf{Z} V\right)$. Hence, the representability of $\Delta_{X / S}$ in general reduces to the special case $S=\operatorname{Spec} \mathbf{Z}$. Moreover, as we just saw, to handle this case it suffices to prove representability of pullbacks $\Delta_{X}^{-1}(V \times W)=V \times{ }_{X} W$ for any pair of maps $v: V \rightarrow X$ and $w: W \rightarrow X$ from schemes $V$ and $W$. By working Zariski-locally on $W$ and then on $V$ we may assume each is affine. Now we shall adapt the proof of [RG, I, 5.7.2] (which is the case when one of $V$ or $W$ is the cover of $X$ in an étale quotient presentation with a representable sheaf of relations).

Fix an étale quotient presentation in schemes $R \rightrightarrows U$ for $X$, as in the definition of $X$ as an algebraic space. Since $U \rightarrow X$ is a surjection for the étale topology and $V$ is an affine scheme, there is an étale affine cover $V^{\prime} \rightarrow V$ fitting into a commutative diagram

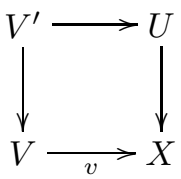

Since $U \times_{X} W$ is a scheme (by [RG, I, 5.7.2]), $V^{\prime} \times_{X} W=V^{\prime} \times_{U}\left(U \times_{X} W\right)$ is also a scheme. If we let $V^{\prime \prime}=V^{\prime} \times_{V} V^{\prime}$ then there is a canonical map $V^{\prime \prime} \rightarrow X$ factoring through either of the two evident maps $V^{\prime \prime} \rightrightarrows U$, and using either of these shows similarly that $V^{\prime \prime} \times_{X} W$ is a scheme. The fiber square of the sheaf quotient map $V^{\prime} \times_{X} W \rightarrow V \times_{X} W$ is identified with the étale equivalence relation in schemes

$$
V^{\prime \prime} \times_{X} W \rightrightarrows V^{\prime} \times_{X} W
$$

which can be interpreted as a descent datum on the $V^{\prime}$-scheme $V^{\prime} \times_{X} W$ relative to the étale covering map of affine schemes $V^{\prime} \rightarrow V$. Hence, our problem is to prove effectivity of this descent problem in the category of $V$-schemes.

The map of schemes $V^{\prime} \times_{X} W \rightarrow V^{\prime} \times_{\text {Spec }} \mathbf{z} W$ with affine target is separated (even a monomorphism), so $V^{\prime} \times{ }_{X} W$ is a separated scheme. Since the maps in (A.1.1.1) are quasi-compact étale surjections, it follows from separatedness of $V^{\prime} \times_{X} W$ that we can cover $V^{\prime} \times_{X} W$ by quasi-compact open subschemes which are stable under the descent datum over $V^{\prime} \rightarrow V$. Now we can argue as in the proof of [RG, I, 5.7.2]: since étale descent is effective for schemes that are quasi-affine over the base [SGA1, VIII, 7.9], it suffices to show that every quasi-compact open subscheme of $V^{\prime} \times_{X} W$ is quasi-affine. Any quasi-finite separated map of schemes is quasi-affine [EGA, $\mathrm{IV}_{4}, 18.12 .12$ ], so it suffices to show that the separated scheme $V^{\prime} \times_{X} W$ admits a locally quasi-finite morphism to an affine scheme. The map of schemes $V^{\prime} \times_{X} W=V^{\prime} \times_{U}\left(U \times_{X} W\right) \rightarrow V^{\prime} \times_{U} W$ is étale since $U \times{ }_{X} W \rightarrow W$ is étale (by [RG, I, 5.7.2]), and the map $V^{\prime} \times_{U} W \rightarrow V^{\prime} \times{ }_{\text {Spec }} \mathbf{Z} W$ with affine target is an immersion since it is a base change of $\Delta_{U}$.

Corollary A.1.2. If $R \rightrightarrows U$ is an étale equivalence relation in algebraic spaces then the quotient $X=U / R$ is an algebraic space.

Proof. Let $U^{\prime} \rightarrow U$ be an étale cover by a separated scheme, so $X=U^{\prime} / R^{\prime}$ where $R^{\prime}=U^{\prime} \times_{X} U^{\prime}=$ $U^{\prime} \times_{U, p_{1}} R \times_{p_{2}, U} U^{\prime}$ is clearly an algebraic space. The natural maps of algebraic spaces $R^{\prime} \rightrightarrows U^{\prime}$ are easily checked to be étale, so we just need to check that $R^{\prime}$ is a scheme. Since $R^{\prime} \rightarrow U^{\prime} \times_{\operatorname{Spec}} \mathbf{Z} U^{\prime}$ is a monomorphism, hence separated, $R^{\prime}$ is a separated algebraic space (since we chose $U^{\prime}$ to be separated). Thus, $p_{1}^{\prime}: R^{\prime} \rightarrow U^{\prime}$ is 
a separated étale map from an algebraic space to a (separated) scheme. It then follows from [K, II, Cor. 6.16] that $R^{\prime}$ is a scheme.

The "topological invariance" of the étale site of a scheme holds more generally for algebraic spaces:

Proposition A.1.3. Let $X$ be an algebraic space. The functor $E \rightsquigarrow E_{\mathrm{red}}=E \times_{X} X_{\mathrm{red}}$ from the category of algebraic spaces étale over $X$ to the category of algebraic spaces étale over $X_{\text {red }}$ is an equivalence.

In particular, the pullback and pushforward functors between étale topoi $\operatorname{Ét}(X)$ and Ét( $\left(X_{\text {red }}\right)$ are naturally inverse to each other.

Proof. Let $U \rightarrow X$ be an étale cover by a scheme and let $R=U \times_{X} U$, so $R$ is also a scheme. Since $U \rightarrow X$ is étale, $U_{\text {red }}=U \times_{X} X_{\text {red }}$ and $R_{\text {red }}=U_{\text {red }} \times_{X_{\text {red }}} U_{\text {red }}$. Hence, $R_{\text {red }} \rightrightarrows U_{\text {red }}$ is an étale chart for $X_{\text {red }}$.

For any algebraic space $E$ equipped with a map $E \rightarrow X$, we may identify $E$ with the quotient of the étale equivalence relation in algebraic spaces $R \times_{X} E \rightrightarrows U \times_{X} E$, and in case $E \rightarrow X$ is étale we see that $E_{\text {red }}$ is the quotient of the analogous equivalence relation

$$
R_{\text {red }} \times_{X_{\text {red }}} E_{\text {red }} \rightrightarrows U_{\text {red }} \times_{X_{\text {red }}} E_{\text {red }}
$$

By Corollary A.1.2, the category of algebraic spaces is stable under the formation of quotients by arbitrary étale equivalence relations. Thus, since the maps $R \rightrightarrows U$ are étale and pull back to the maps $R_{\text {red }} \rightrightarrows U_{\text {red }}$, we easily reduce to proving the lemma for $U$ and $R$ in place of $X$. That is, we can assume that $X$ is a scheme.

The equivalence result for categories of schemes étale over $X$ and $X_{\text {red }}$ is [EGA, $\left.\mathrm{IV}_{4}, 18.1 .2\right]$. Thus, if $E_{0}$ is an algebraic space étale over $X_{\text {red }}$ and $U_{0} \rightarrow E_{0}$ is an étale scheme cover then $R_{0}=U_{0} \times_{E_{0}} U_{0}$ is a scheme

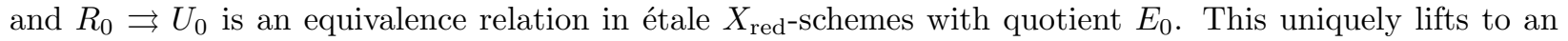
equivalence relation $R \rightrightarrows U$ in étale $X$-schemes, and it is clearly an étale equivalence relation. Hence, the quotient $E=U / R$ makes sense as an algebraic space étale over $X$, and $E \times_{X} X_{\text {red }} \simeq U_{0} / R_{0}=E_{0}$. Thus, we have proved essential surjectivity.

For full faithfulness, let $E$ and $E^{\prime}$ be algebraic spaces étale over $X$. To show that the map $\operatorname{Hom}_{X}\left(E, E^{\prime}\right) \rightarrow$ $\operatorname{Hom}_{X_{\text {red }}}\left(E_{\text {red }}, E_{\text {red }}^{\prime}\right)$ is bijective, by using an étale scheme chart for $E$ we may easily reduce to the case when $E$ is a scheme. That is, it suffices to prove that $E^{\prime}(V) \rightarrow E_{\text {red }}^{\prime}\left(V_{\text {red }}\right)$ is bijective for any étale $X$-scheme $V$ and any algebraic space $E^{\prime}$ over $X$. Let $j: X_{\text {red }} \rightarrow X$ denote the canonical map. The functors $j_{*}$ and $j^{*}$ between small étale sites are inverse equivalences of categories (essentially due to the equivalence result for schemes [EGA, $\left.\mathrm{IV}_{4}, 18.1 .2\right]$ that we already used), and if $V \rightarrow X$ is an étale map of schemes we naturally have

$$
j^{*}(V) \simeq V \times_{X} X_{\text {red }}=V_{\text {red }}
$$

as sheaves on the small étale site of $X_{\text {red }}$. Thus, it suffices to show that the natural map $E_{\text {red }}^{\prime} \rightarrow j^{*}\left(E^{\prime}\right)$ of sheaves on the small étale site of $X_{\text {red }}$ is an isomorphism. But $j^{*}$ is an exact functor, so by using an étale scheme chart for $E^{\prime}$ we reduce to the case when $E^{\prime}$ is a scheme, in which case the map of interest is identified with the map in A.1.3.1 that is an isomorphism.

A.2. Topology of algebraic spaces. We briefly review a few facts about the topology of quasi-separated algebraic spaces. Rydh has recently developed a theory of points and topological spaces associated to general algebraic spaces.

Definition A.2.1. Let $S$ be an algebraic space. It is noetherian if it is quasi-separated and admits an étale cover by a noetherian scheme. It is locally noetherian if it admits a Zariski-open covering by noetherian open subspaces.

It is clear that any locally noetherian algebraic space is quasi-separated. In [K, II, 8.5] the irreducible component decomposition of noetherian algebraic spaces $S$ is developed, assuming $S$ is (quasi-separated and) locally separated in the sense that the map $\Delta_{S}$ is a quasi-compact immersion. The theory of irreducible components is developed in [LMB] for quasi-separated Artin stacks. (Quasi-separatedness is a running assumption throughout [LMB, along with separatedness of the diagonal.) More specifically, specializing LMB, 5.7.2] to the case of algebraic spaces, one gets: 
Lemma A.2.2. Let $S$ be a quasi-separated algebraic space. Every irreducible closed set in $|S|$ has a unique generic point, and if $f: S^{\prime} \rightarrow S$ is an étale surjective map of quasi-separated algebraic spaces then every irreducible component of $S$ has generic point of the form $f\left(s^{\prime}\right)$ for some generic point $s^{\prime}$ of $S^{\prime}$.

We do not know if Lemma A.2.2 holds without quasi-separatedness.

The following proposition is known more generally for quasi-separarted Artin stacks, but we include a proof for the convenience of the reader.

Proposition A.2.3. Let $S$ be a quasi-separated algebraic space. Its irreducible components constitute the unique collection $\left\{S_{i}\right\}$ of pairwise distinct irreducible and reduced closed subspaces of $S$ such that they cover $S$ and no $\left|S_{i}\right|$ is contained in $\cup_{j \neq i}\left|S_{j}\right|$. If $V$ is open in $S$ then the corresponding collection for $V$ is the collection of those $V \cap S_{i}$ that are non-empty.

If $S$ is locally noetherian then $\left\{S_{i}\right\}$ is Zariski-locally finite and the normalization of $S_{\mathrm{red}}$ is the disjoint union $\amalg \widetilde{S}_{i}$ where $\widetilde{S}_{i}$ is the normalization of $S_{i}$ and each $\widetilde{S}_{i}$ is irreducible. In particular, if $S$ is normal and locally noetherian then $\left\{S_{i}\right\}$ is its set of connected components.

Proof. To show that an irreducible component of $S$ is not covered (as a topological space) by the union of the other irreducible components of $S$, it suffices to show that if $S$ is irreducible then $|S|$ is not covered by proper closed subsets. The existence of a generic point as in Lemma A.2.2 makes this clear. It is likewise clear via generic points that if $V$ is open in $S$ and $\left\{S_{i}\right\}$ is the set of irreducible components of $S$ then the non-empty $V \cap S_{i}$ 's are the irreducible components of $V$ (as topological closure preserves irreducibility).

For the uniqueness claim concerning the collection $\left\{S_{i}\right\}$, consider any collection $\left\{S_{i}^{\prime}\right\}$ of irreducible and reduced closed subspaces of $S$ such that the $\left|S_{i}^{\prime}\right|$ 's cover $|S|$ and each $\left|S_{i}^{\prime}\right|$ is not contained in the union of the $\left|S_{j}^{\prime}\right|$ 's for $j \neq i$. Each $S_{i}^{\prime}$ must be an irreducible component, since if $S_{i}^{\prime}$ is strictly contained in another irreducible and reduced closed subspace $Z$ then the generic point of $Z$ cannot lie in $S_{i}^{\prime}$ and so lies in $S_{j}^{\prime}$ for some $j \neq i$, a contradiction since then $S_{i}^{\prime} \subseteq Z \subseteq S_{j}^{\prime}$ by closedness of each $S_{j}^{\prime}$ in $S^{\prime}$. Moreover, the generic point of each irreducible component $Y$ in $S$ lies in some $S_{i}^{\prime}$ and hence $Y=S_{i}^{\prime}$ by maximality for $Y$. This completes the proof of uniqueness.

In the locally noetherian case the topological space $|S|$ is locally noetherian, so $\left\{S_{i}\right\}$ is Zariski-locally finite in $S$. Consider the normalization $\widetilde{S}$ of $S_{\text {red }}$. The description of its irreducible components is known in the scheme case, and so follows in general by working over a dense open subspace $U \subseteq S$ that is a scheme $\mathbb{K}$, II, 6.8] (with the normalization $\widetilde{U}$ likewise a dense open subspace of $\widetilde{S}$ ). The irreducible components of $\widetilde{U}$ are its connected components, so to check that the irreducible components of $\widetilde{S}$ are pairwise disjoint it suffices to show that idemponents on $\widetilde{U}$ uniquely extend to idempotents on $\widetilde{S}$. More generally, if $N$ is a normal algebraic space and if $V$ is a dense open subspace, then idempotents of $V$ uniquely extend to idempotents of $N$. Indeed, to prove this it suffices to work étale-locally on $N$, so we reduce to the known case when $N$ is a normal scheme.

A.3. Properties of inverse limits of algebraic spaces. The results in this section extend to the case of algebraic spaces a variety of known facts concerning inverse limits of schemes.

Proposition A.3.1. Let $X \rightarrow Y$ be a map locally of finite presentation between algebraic spaces such that $X$ and $Y$ are covered by Zariski-open subspaces that are quasi-separated. If $\left\{S_{\alpha}\right\}$ is an inverse system of algebraic spaces over $Y$ with affine transition maps and each $S_{\alpha}$ is qcqs then

is bijective.

$$
\underset{\lim _{\longrightarrow}}{\operatorname{Hom}_{Y}}\left(S_{\alpha}, X\right) \rightarrow \operatorname{Hom}_{Y}\left(\lim _{\longleftarrow} S_{\alpha}, X\right)
$$

In the case of schemes the Zariski covering hypotheses on $X$ and $Y$ are always satisfied, so when all objects (including the $S_{\alpha}$ are schemes this proposition is part of [EGA, $\left.\mathrm{IV}_{3}, 8.14 .2(\mathrm{~b})\right]$.

Proof. The first part of the proof consists of some tedious but mechanical arguments to reduce to the case when $X$ is qcqs and $Y$ and all $S_{\alpha}$ are affine. Then the real argument can begin.

Since any particular $S_{\alpha_{0}}$ is quasi-compact, so a Zariski-open covering of $Y$ has pullback to $S_{\alpha_{0}}$ admitting a finite quasi-compact open subcover, it is straightforward to reduce to general problem over $Y$ to the general 
problem over each member of a Zariski-open covering of $Y$. Hence, we can assume that $Y$ is quasi-separated, and then repeating the same argument reduces us to the case when $Y$ is qcqs. Hence, $Y$ admits an étale cover $Y^{\prime} \rightarrow Y$ by an affine scheme $Y^{\prime}$, and this covering map is quasi-compact (since $Y$ is qcqs) and separated. An elementary étale descent argument thereby reduces our problem to the case when $Y$ is affine.

Let $U_{\alpha_{0}} \rightarrow S_{\alpha_{0}}$ be an étale cover by an affine scheme for some $\alpha_{0}$ and let $R_{\alpha_{0}}=U_{\alpha_{0}} \times S_{\alpha_{0}} U_{\alpha_{0}}$, so $R_{\alpha_{0}}$ is a quasi-compact and separated scheme. We may and do assume $\alpha \geq \alpha_{0}$ for all $\alpha$. Define $R_{\alpha} \rightrightarrows U_{\alpha}$ and $R \rightrightarrows U$ by base change along $S_{\alpha} \rightarrow S_{\alpha_{0}}$ and $S:=\lim _{\leftarrow} S_{\alpha} \rightarrow S_{\alpha_{0}}$, so these are respective étale charts for $S_{\alpha}$ and $S$. It is easy to reduce to the analogous bijectivity claims for the inverse systems $\left\{U_{\alpha}\right\}$ and $\left\{R_{\alpha}\right\}$. In particular, we may assume that all $S_{\alpha}$ are schemes, and then even quasi-compact and separated (and even affine).

With all $S_{\alpha}$ now quasi-compact separated schemes, any quasi-compact open subscheme of lim $S_{\alpha} \operatorname{descends}$ to a quasi-compact open subscheme of some $S_{\alpha_{0}}$. Thus, if $\left\{X_{i}\right\}$ is any Zariski-open covering of $X$ and we can solve the problem for each $X_{i}$ separately (for any inverse system $\left\{S_{\alpha}\right\}$ of quasi-compact and separated schemes equipped with a compatible map to $Y$ ) then it is straightforward to use quasi-compact open refinements of pullbacks of the Zariski-covering $\left\{X_{i}\right\}$ get the result for $X$. But we assumed that $X$ has a Zariski-open covering by quasi-separated algebraic spaces, so we may arrange that $X$ is qcqs. Hence, $X \rightarrow Y$ is finitely presented. We may also now run through the localization argument on the $S_{\alpha}$ 's to reduce to the case when all $S_{\alpha}$ are affine. Keep in mind that we also reduced to the case when $Y$ is affine.

Consider a $Y$-map $f: S \rightarrow X$. Let $V \rightarrow X$ be an étale cover by an affine scheme, so this covering map is quasi-compact (since $X$ is quasi-separated) and separated (since $V$ is separated). Hence, $V$ is finitely presented over $Y$ and the étale scheme cover $W:=f^{-1}(V) \rightarrow S$ is finitely presented (i.e., qcqs). It follows that $W \rightarrow S$ descends to a qcqs étale scheme cover $W_{\alpha_{0}} \rightarrow S_{\alpha_{0}}$ for some $\alpha_{0}$. The pullbacks $W_{\alpha}=W_{\alpha_{0}} \times S_{\alpha_{0}} S_{\alpha}$ form an inverse system of $Y$-schemes, with affine transition maps, so since $V$ is a scheme that is finitely presented over $Y$ it follows that the natural map

$$
\underset{\lim }{\longrightarrow} \operatorname{Hom}_{Y}\left(W_{\alpha}, V\right) \rightarrow \operatorname{Hom}_{Y}(W, V)
$$

is bijective by EGA $\left.\mathrm{IV}_{3}, 8.14 .2(\mathrm{~b})\right]$. Thus, the natural $Y$-map $h: W=f^{-1}(V) \rightarrow V$ factors through a $Y$-map $h_{\alpha_{1}}: W_{\alpha_{1}} \rightarrow V$ for some large $\alpha_{1}$.

The pair of maps $h_{\alpha_{1}} \circ p_{1}, h_{\alpha_{1}} \circ p_{2}$ in $\operatorname{Hom}_{Y}\left(W_{\alpha_{1}} \times_{S_{\alpha_{1}}} W_{\alpha_{1}}, V\right)$ may not coincide, but they do yield the same map $h \circ p_{1}=h \circ p_{2}$ after composing back to $W \times_{S} W=f^{-1}\left(V \times_{X} V\right)$. Thus, since $\left\{W_{\alpha} \times_{S_{\alpha}} W_{\alpha}\right\}$ is an inverse system of qcqs schemes with affine transition maps (over $Y$ ) and limit $W \times{ }_{S} W$, by the injectivity of the map

$$
\underset{\lim _{Y}}{\longrightarrow} \operatorname{Hom}_{Y}\left(W_{\alpha} \times_{S_{\alpha}} W_{\alpha}, V\right) \simeq \operatorname{Hom}_{Y}\left(W \times_{S} W, V\right)
$$

it follows that if we enlarge $\alpha_{1}$ enough then $h_{\alpha_{1}} \circ p_{1}=h_{\alpha_{1}} \circ p_{2}$. That is, $h_{\alpha_{1}}$ factors through a $Y$-map $f_{\alpha_{1}}: S_{\alpha_{1}} \rightarrow V$. It suffices to check that this induces the original map $f: S \rightarrow V$ after composing with $S \rightarrow S_{\alpha_{1}}$. Such an equality may be checked after composing with the étale covering map $W \rightarrow S$, so we are done because the diagram

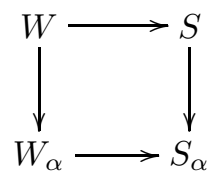

commutes for all $\alpha$ (due to how $W_{\alpha}$ was defined).

Corollary A.3.2. Let $\left\{S_{\alpha}\right\}$ be an inverse system of qcqs algebraic spaces with affine transition maps and limit $S$. Let $\left\{X_{\alpha}\right\}$ and $\left\{Y_{\alpha}\right\}$ be compatible cartesian systems of finitely presented algebraic spaces over $\left\{S_{\alpha}\right\}$, and let $X=\lim X_{\alpha}$ and $Y=\lim Y_{\alpha}$.

The natural map

$$
\underset{\lim }{\longrightarrow} \operatorname{Hom}_{S_{\alpha}}\left(X_{\alpha}, Y_{\alpha}\right) \rightarrow \operatorname{Hom}_{S}(X, Y)
$$

is bijective. 
In Corollary A.3.5 we will address the descent of properties of morphisms through such limits. We postpone it because the verification of the properties requires an additional result given in Proposition A.3.4 below.

Proof. Fix some $\alpha_{0}$ and consider only $\alpha \geq \alpha_{0}$ without loss of generality. Since $Y_{\alpha}=S_{\alpha} \times S_{\alpha_{0}} Y_{\alpha_{0}}$ and $Y=S \times{ }_{\alpha_{\alpha_{0}}} Y_{\alpha_{0}}$, the map of interest may be expressed as

$$
\underset{\lim _{\longrightarrow}}{\operatorname{Hom}_{S_{\alpha_{0}}}}\left(X_{\alpha}, Y_{\alpha_{0}}\right) \rightarrow \operatorname{Hom}_{S_{\alpha_{0}}}\left(\lim _{\longleftarrow} X_{\alpha}, Y_{\alpha_{0}}\right) \text {. }
$$

Thus, the desired bijectivity is a consequence of Proposition A.3.1.

Lemma A.3.3. Let $\left\{S_{\alpha}\right\}$ be an inverse system of qcqs algebraic spaces with affine transition maps. Let $S=\lim S_{\alpha}$, and assume that some $S_{\alpha^{\prime}}$ is affine over an algebraic space of finite presentation over $\mathbf{Z}$.

If $\overleftarrow{S}$ is a scheme (resp. affine scheme) then so is $S_{\alpha}$ for sufficiently large $\alpha$.

The hypothesis on some $S_{\alpha^{\prime}}$ is satisfied by all $S_{\alpha}$ when they are schemes, by absolute noetherian approximation for qcqs schemes [TT, Thm. C.9]. The proof of absolute noetherian approximation for qcqs algebraic spaces in 3.1 requires Lemma A.3.3 in the special case that all $S_{\alpha}$ are of finite presentation over $\mathbf{Z}$, but once that result is proved in general then the hypothesis on some $S_{\alpha^{\prime}}$ in Lemma.3.3 may be dropped (as it is then satisfied for any $\left.\alpha^{\prime}\right)$.

Proof. Fix an $\alpha^{\prime}$ as in the statement of the lemma, and consider only $\alpha \geq \alpha^{\prime}$ without loss of generality. In particular, all $S_{\alpha}$ are affine over an algebraic space $\mathscr{S}$ of finite presentation over Z. When the $S_{\alpha}$ 's are all schemes, by absolute noetherian approximation for qcqs schemes [TT, Thm. C.9] we may choose $\mathscr{S}$ to be a scheme; note that $\mathscr{S}$ is an auxiliary device, so changing it at the outset is not a problem. (Even when the $S_{\alpha}$ are all schemes and $S$ is affine, our desired conclusion that $S_{\alpha}$ is affine for all large $\alpha$ is slightly more general than [TT, Prop. C.6] in the sense that the argument there assumes the $S_{\alpha}$ are all finite type over $\mathbf{Z}$, or over some noetherian ring.)

Fix some $\alpha_{0}$ and let $U_{\alpha_{0}} \rightarrow S_{\alpha_{0}}$ be an étale scheme cover with $U_{\alpha_{0}}$ a qcqs scheme (e.g., affine), and define $R_{\alpha_{0}}=U_{\alpha_{0}} \times S_{\alpha_{0}} U_{\alpha_{0}}$, so $R_{\alpha_{0}}$ is a qcqs scheme since $S_{\alpha_{0}}$ is quasi-separated and $U_{\alpha_{0}}$ is quasi-compact. Define $R_{\alpha} \rightrightarrows U_{\alpha}$ and $R \rightrightarrows U$ by base change to $S_{\alpha}$ and $S$ respectively, so these are étale scheme charts for $S_{\alpha}$ and $S$ respectively. Let $p: U \rightarrow S$ be the covering map, and $p_{1}, p_{2}: R \rightrightarrows U$ be the two projections.

We claim (exactly as is known for inverse systems of qcqs schemes with affine transition maps) that if $V \subseteq S$ is a quasi-compact open subspace then $V$ descends to a quasi-compact open subspace $V_{\alpha_{0}} \subseteq S_{\alpha_{0}}$ for some $\alpha_{0}$, and that if $V_{\alpha_{0}}^{\prime} \subseteq S_{\alpha_{0}}$ is another such descent then the pullbacks of $V_{\alpha_{0}}$ and $V_{\alpha_{0}}^{\prime}$ to $S_{\alpha}$ coincide for sufficiently large $\alpha \geq \alpha_{0}$.

To prove these claims, consider the quasi-compact open subscheme $W:=p^{-1}(V) \subseteq U$. Since $\left\{U_{\alpha}\right\}$ is an inverse system of qcqs schemes with affine transition maps and limit $U$, certainly $W$ descends to a quasicompact open subscheme $W_{\alpha_{0}} \subseteq U_{\alpha_{0}}$ for some $\alpha_{0}$. The two open subschemes $p_{1, \alpha_{0}}^{-1}\left(W_{\alpha_{0}}\right)$ and $p_{2, \alpha_{0}}^{-1}\left(W_{\alpha_{0}}\right)$ in $R_{\alpha_{0}}$ have pullbacks $p_{1}^{-1}(W)$ and $p_{2}^{-1}(W)$ in $R=\lim _{\longleftarrow} R_{\alpha}$ which coincide. Since $\left\{R_{\alpha}\right\}$ is an inverse system of qcqs schemes with affine transition maps, by increasing $\alpha_{0}$ we can ensure that the two pullbacks of $W_{\alpha_{0}}$ into $R_{\alpha_{0}}$ coincide, so $W_{\alpha_{0}}$ descends to a quasi-compact open subspace $V_{\alpha_{0}} \subseteq S_{\alpha_{0}}$, and this clearly descends $V$. If $V_{\alpha_{0}}^{\prime}$ is another such descent then the desired equality $V_{\alpha}=V_{\alpha}^{\prime}$ of pullbacks into $S_{\alpha}$ for sufficiently large $\alpha$ can be checked by working in the inverse system $\left\{U_{\alpha}\right\}$ of qcqs étale scheme covers of $\left\{S_{\alpha}\right\}$, so we are reduced to the known scheme case.

Choose a finite covering of the scheme $S$ by affine open subschemes $\left\{V_{1}, \ldots, V_{n}\right\}$. By what we just proved, for sufficiently large $\alpha_{0}$ this descends to a finite collection of quasi-compact open subspaces $\left\{V_{1, \alpha_{0}}, \ldots, V_{n, \alpha_{0}}\right\}$ in $S_{\alpha_{0}}$, and by taking $\alpha_{0}$ big enough it constitutes a covering. For each $1 \leq i \leq n$, the resulting inverse system $\left\{V_{i, \alpha}\right\}_{\alpha \geq \alpha_{0}}$ of qcqs algebraic spaces with affine transition maps has limit $V_{i}$ that is an affine scheme, and $\left\{V_{1, \alpha}, \ldots, V_{n, \alpha}\right\}$ is an open cover of $S_{\alpha}$. Thus, $S_{\alpha}$ is a scheme if each $V_{i, \alpha}$ is a scheme, so for each fixed $i$ we may rename $V_{i}$ as $S$ and $V_{i, \alpha}$ as $S_{\alpha}$ to reduce to the case when $S$ is an affine scheme, provided of course that we prove the stronger conclusion that $S_{\alpha}$ is an affine scheme for sufficiently large $\alpha$.

Recall that $\left\{S_{\alpha}\right\}$ is an inverse system of objects that are affine over an algebraic space $\mathscr{S}$ of finite presentation over $\mathbf{Z}$, and that when all $S_{\alpha}$ are schemes we may take $\mathscr{S}$ to be a scheme. Since the map 
$S \rightarrow S_{\alpha}$ is affine, we can form its schematic image $S_{\alpha}^{\prime} \subseteq S_{\alpha}$ for all $\alpha$, and clearly $\left\{S_{\alpha}^{\prime}\right\}$ is an inverse system with schematically dominant and affine transition maps such that the natural map $S \rightarrow \lim S_{\alpha}^{\prime}$ is an

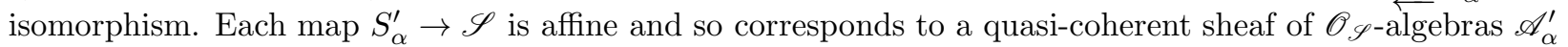
with injective transition maps such that $S$ corresponds to $\mathscr{A}=\lim _{\mathfrak{A}} \mathscr{A}_{\alpha}^{\prime}$. By [K] III, Thm. 1.1, Cor. 1.2], each $\mathscr{A}_{\alpha}^{\prime}$ is the direct limit of its coherent $\mathscr{O}_{\mathscr{S}}$-submodules. Passing to the $\mathscr{O}_{\mathscr{S}}$-subalgebras generated by these,

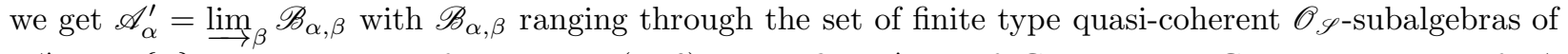
$\mathscr{A}_{\alpha}^{\prime}$. Let $\{\gamma\}$ denote the set of such pairs $(\alpha, \beta)$ and define $\gamma^{\prime} \geq \gamma$ if $\mathscr{B}_{\gamma^{\prime}}$ contains $\mathscr{B}_{\gamma}$ as subsheaves of $\mathscr{A}$. Hence $S=\lim _{\zeta} T_{\gamma}$ over $\mathscr{S}$ with $T_{\gamma}=\operatorname{Spec}_{\mathscr{S}}\left(\mathscr{B}_{\gamma}\right)$.

By Proposition A.3.1 applied to the finitely presented map $T_{\gamma} \rightarrow \mathscr{S}$ and the inverse system $\left\{S_{\alpha}\right\}$ over $\mathscr{S}$ with limit $S$, for each $\gamma_{0}$ the canonical $\mathscr{S}$-map $S \rightarrow T_{\gamma_{0}}$ factors through an $\mathscr{S}$-map $S_{\alpha} \rightarrow T_{\gamma_{0}}$ for some sufficiently large $\alpha$ (depending on $\gamma_{0}$ ). But $S_{\alpha}$ and $T_{\gamma_{0}}$ are each affine over $\mathscr{S}$, so any $\mathscr{S}$-map between them is automatically affine. Thus, if $T_{\gamma_{0}}$ is affine for some $\gamma_{0}$ then $S_{\alpha}$ is affine for all large $\alpha$, as desired. It is therefore permissible to replace $\left\{S_{\alpha}\right\}$ with $\left\{T_{\gamma}\right\}$, so we thereby reduce to the case when all $S_{\alpha}$ are of finite presentation over $\mathbf{Z}$. This reduction step preserves the property that all $S_{\alpha}$ are schemes if we began in that case and took $\mathscr{S}$ to be a scheme (as we may when all $S_{\alpha}$ are schemes). Now that all $S_{\alpha}$ are of finite presentation over $\mathbf{Z}$, if they are all schemes then $S$ being affine forces $S_{\alpha}$ to be affine for all large $\alpha$ by TT, Prop. C.6]. Thus, in the original problem, if the $S_{\alpha}$ are schemes and $S$ is affine then we have deduced the desired stronger conclusion that the $S_{\alpha}$ are affine for all large $\alpha$. (In other words, we have slightly generalized [TT, Prop. C.6] by eliminating the finite presentation hypotheses there.)

Consider the original general problem, with $S$ a scheme and each $S_{\alpha}$ just an algebraic space. To conclude that the $S_{\alpha}$ are schemes for large $\alpha$, we have already reduced ourselves to the case when $S$ is an affine scheme and the $S_{\alpha}$ are algebraic spaces of finite presentation over $\mathbf{Z}$. In this case we want to conclude that once again the $S_{\alpha}$ are affine for all large $\alpha$. Since Proposition A.3.1 is available in the category of algebraic spaces, we may use it (with $Y=\operatorname{Spec} \mathbf{Z}$ ) in place of [TT, C.5] to make the proof of the scheme case in [TT, Prop. C.6] work verbatim with each $S_{\alpha}$ an algebraic space of finite presentation over $\mathbf{Z}$.

The following result is useful for both descent through limits of algebraic spaces and relating such descents to limit procedures with schemes.

Proposition A.3.4. Let $\left\{S_{\alpha}\right\}$ be an inverse system of qcqs algebraic spaces with affine transition maps and limit $S$. Assume that some $S_{\alpha^{\prime}}$ is affine over an algebraic space of finite presentation over $\mathbf{Z}$.

For any finitely presented morphism $T \rightarrow S$ from an algebraic space (resp. scheme) $T$, there exists an $\alpha_{0}$ and a finitely presented map $T_{\alpha_{0}} \rightarrow S_{\alpha_{0}}$ from an algebraic space (resp. scheme) $T_{\alpha_{0}}$ such that $T \simeq T_{\alpha_{0}} \times_{S_{\alpha_{0}}} S$ over $S$, so the natural map

$$
T \rightarrow \lim _{\alpha \geq \alpha_{0}} T_{\alpha}
$$

is an isomorphism with $T_{\alpha}:=T_{\alpha_{0}} \times_{S_{\alpha_{0}}} S_{\alpha}$.

The hypothesis that some $S_{\alpha^{\prime}}$ is affine over an algebraic space of finite presentation over $\mathbf{Z}$ is always satisfied when the $S_{\alpha}$ are schemes, by absolute noetherian approximation for qcqs schemes [TT, Thm. C.9]. This hypothesis will be satisfied in general once absolute noetherian approximation for qcqs algebraic spaces is proved in 3.1 The proof of this latter approximation result requires Proposition A.3.4 only in the special case that all $S_{\alpha}$ are of finite presentation over $\mathbf{Z}$, so there is no circularity.

Note also that if $\left\{S_{\alpha}\right\}$ has schematically dominant transition maps and $T \rightarrow S$ is flat then $\left\{T_{\alpha}\right\}$ has schematically dominant transition maps for sufficiently large indices because the quasi-compact map $T_{\alpha} \rightarrow S_{\alpha}$ is flat for large $\alpha$ (as we see by working étale-locally on $S_{\alpha_{0}}$ and $T_{\alpha_{0}}$ to reduce to the known scheme case).

Proof. Fix some $\alpha_{0}$ and choose an étale chart in schemes

$$
R_{0}:=U_{0} \times_{S_{\alpha_{0}}} U_{0} \rightrightarrows U_{0} \rightarrow S_{\alpha_{0}}
$$

with $U_{0}$ qcqs étale over $S_{\alpha_{0}}$ (e.g., an affine scheme), so both $U_{0}$ and $R_{0}$ are qcqs schemes. For any $\alpha \geq \alpha_{0}$ we define $U_{\alpha}=U_{0} \times_{S_{\alpha_{0}}} S_{\alpha}$ and $R_{\alpha}=R_{0} \times_{S_{\alpha_{0}}} S_{\alpha}$, so $R_{\alpha} \rightrightarrows U_{\alpha}$ is naturally an étale scheme chart for $S_{\alpha}$ compatible with change in $\alpha$ and moreover the inverse systems $\left\{U_{\alpha}\right\}$ and $\left\{R_{\alpha}\right\}$ have affine transition maps. 
The corresponding inverse limits $U$ and $R$ provide an étale equivalence relation $R \rightrightarrows U$ over $S$ obtained by base change of any $R_{\alpha} \rightrightarrows U_{\alpha}$ along $S \rightarrow S_{\alpha}$. It is clear that $R \rightarrow U \times_{S} U$ is an isomorphism, so $R \rightrightarrows U$ is an étale chart for the algebraic space $S$. Note that $U \rightarrow S$ is a qcqs étale (i.e., finitely presented étale) map.

Now we turn our attention to the finitely presented map $T \rightarrow S$. First we consider the case when $T$ is a scheme, and then we bootstrap this to the case when $T$ is an algebraic space. The pullback $U^{\prime}=T \times{ }_{S} U$ is quasi-compact and étale over $T$, and it is a scheme since $T$ and $U$ are schemes. Likewise, $R^{\prime}=T \times{ }_{S} R$ is a quasi-compact scheme and $R^{\prime} \rightrightarrows U^{\prime}$ is an étale equivalence relation with $R^{\prime}=U^{\prime} \times_{T} U^{\prime}$, so $U^{\prime} / R^{\prime}=T$. The natural étale $S$-map $q: U^{\prime} \rightarrow U=\lim U_{\alpha}$ is a qcqs map, hence it is of finite presentation, so since $U^{\prime}$ and the $U_{\alpha}$ 's are schemes we may use standard limit results for schemes to increase $\alpha_{0}$ so that there exists a qcqs étale map of schemes $U_{\alpha_{0}}^{\prime} \rightarrow U_{\alpha_{0}}$ which descends $q$. We use this descended map to define $U_{\alpha_{0}}^{\prime} \rightarrow S_{\alpha_{0}}$, and define $U_{\alpha}^{\prime}=U_{\alpha_{0}}^{\prime} \times_{S_{\alpha_{0}}} S_{\alpha}$ for all $\alpha \geq \alpha_{0}$. Clearly $\left\{U_{\alpha}^{\prime}\right\}_{\alpha \geq \alpha_{0}}$ has affine transition maps (so each $U_{\alpha}^{\prime}$ is a scheme) and $U^{\prime}=\lim U_{\alpha}^{\prime}$ over $U=\lim U_{\alpha}$. By increasing $\alpha_{0}$ if necessary, we may also descend $R^{\prime} \rightarrow R$ to a qcqs étale map of schemes $R_{\alpha_{0}}^{\prime} \rightarrow \overleftarrow{R}_{\alpha_{0}}$ and define the inverse system of schemes $R_{\alpha}^{\prime}=R_{\alpha_{0}}^{\prime} \times_{S_{\alpha_{0}}} S_{\alpha}$ with affine transition maps and limit $R^{\prime}$.

Consider the two étale $S$-maps $p_{1}^{\prime}, p_{2}^{\prime}: R^{\prime} \rightrightarrows U^{\prime}$ over the pair of $S$-maps $p_{1}, p_{2}: R \rightrightarrows U$ between schemes étale over $S$. By Corollary A.3.2 if we increase $\alpha_{0}$ then we can arrange that the maps $p_{1}^{\prime}$ and $p_{2}^{\prime}$ descend to a pair of $S_{\alpha_{0}}$-maps $p_{1, \alpha_{0}}^{\prime}, p_{2, \alpha_{0}}^{\prime}: R_{\alpha_{0}}^{\prime} \rightrightarrows U_{\alpha_{0}}^{\prime}$ over the respective projections $p_{1, \alpha_{0}}$ and $p_{2, \alpha_{0}}$. For $\alpha \geq \alpha_{0}$ and $i \in\{1,2\}$, define the $S_{\alpha}$-map $p_{i, \alpha}^{\prime}: R_{\alpha}^{\prime} \rightarrow U_{\alpha}^{\prime}$ to be the base change of $p_{i, \alpha_{0}}^{\prime}$ along $S_{\alpha} \rightarrow S_{\alpha_{0}}$. Now consider only $\alpha \geq \alpha_{0}$. In particular, each $p_{i, \alpha}^{\prime}$ is a qcqs étale $S_{\alpha}$-map and base change along $S_{\beta} \rightarrow S_{\alpha}$ for $\beta \geq \alpha$ carries $p_{i, \alpha}^{\prime}$ to $p_{i, \beta}^{\prime}$.

We claim that $R_{\alpha}^{\prime} \rightrightarrows U_{\alpha}^{\prime}$ is an étale equivalence relation for sufficiently large $\alpha$. In other words, for large $\alpha$ we claim that the maps $p_{i, \alpha}^{\prime}$ are étale and that the map $R_{\alpha}^{\prime} \rightarrow U_{\alpha}^{\prime} \times_{\operatorname{Spec}} \mathbf{Z} U_{\alpha}^{\prime}$ is a monomorphism which functorially defines an equivalence relation. This latter map factors through the subfunctor $U_{\alpha}^{\prime} \times_{S_{\alpha}} U_{\alpha}^{\prime}$ which is also a scheme, so for the equivalence relation condition it is equivalent to check that the map $\delta_{\alpha}: R_{\alpha}^{\prime} \rightarrow U_{\alpha}^{\prime} \times_{S_{\alpha}} U_{\alpha}^{\prime}$ is a monomorphism defining a functorial equivalence relation for large $\alpha$. Note that $\delta_{\alpha}$ is a finitely presented map since it is an $S_{\alpha}$-map between schemes of finite presentation along over $S_{\alpha}$.

Since $\left\{p_{i, \alpha}^{\prime}\right\}$ is a compatible system of finitely presented maps between inverse systems of qcqs schemes with affine transition maps and the limit map $p_{i}^{\prime}: R^{\prime} \rightarrow U^{\prime}$ is étale, by [EGA, $\left.\mathrm{IV}_{4}, 17.7 .8(\mathrm{ii})\right]$ it follows that $p_{1, \alpha}^{\prime}$ and $p_{2, \alpha}^{\prime}$ are étale for all large $\alpha$. Likewise, $\left\{U_{\alpha}^{\prime} \times_{S_{\alpha}} U_{\alpha}^{\prime}\right\}$ is an inverse system of qcqs schemes with affine transition maps and limit $U^{\prime} \times{ }_{S} U^{\prime}$, and base change of the finitely presented scheme map $\delta_{\alpha}$ along a transition map in this system (resp. along pullback to the limit) is identified with the base change along the corresponding transition map in $\left\{S_{\alpha}\right\}$ (resp. along pullback from $S_{\alpha}$ to $S$ ). Since the limit map $\delta=\left(p_{1}^{\prime}, p_{2}^{\prime}\right): R^{\prime} \rightarrow U^{\prime} \times_{S} U^{\prime}$ is an equivalence relation, in particular it is a monomorphism. Thus, by EGA, $\mathrm{IV}_{4}, 8.10 .5$ (i bis)] the $S_{\alpha}$-maps $\delta_{\alpha}$ are monomorphisms for sufficiently large $\alpha$. Likewise, if $\alpha$ is large enough then the symmetry automorphism $R^{\prime} \simeq R^{\prime}$ over the flip on the scheme $U^{\prime} \times_{S} U^{\prime}$ carrying $\left(p_{1}^{\prime}, p_{2}^{\prime}\right)$ to $\left(p_{2}^{\prime}, p_{1}^{\prime}\right)$ descends to such an automorphism $R_{\alpha}^{\prime} \simeq R_{\alpha}^{\prime}$ over the flip on the scheme $U_{\alpha}^{\prime} \times_{S_{\alpha}} U_{\alpha}^{\prime}$ carrying $\left(p_{1, \alpha}^{\prime}, p_{2, \alpha}^{\prime}\right)$ to $\left(p_{2, \alpha}^{\prime}, p_{1, \alpha}^{\prime}\right)$, so the functorial relation $R_{\alpha}^{\prime}$ on $U_{\alpha}^{\prime}$ is symmetric for sufficiently large $\alpha$. Similarly, the transitivity morphism of schemes $R^{\prime} \times \times_{2}^{\prime}, U^{\prime}, p_{1}^{\prime} R^{\prime} \rightarrow R^{\prime}$ induced by the scheme map $q_{13}: U^{\prime} \times_{S} U^{\prime} \times{ }_{S} U^{\prime} \rightarrow U^{\prime} \times{ }_{S} U^{\prime}$ descends to the $\alpha$-level for sufficiently large $\alpha$, and the diagonal $\Delta_{U_{\alpha}^{\prime} / S_{\alpha}}$ factors through $\delta_{\alpha}$ for sufficiently large $\alpha$. Hence, for some large $\alpha_{1}$ we have that $R_{\alpha}^{\prime}$ defines an equivalence relation on $U_{\alpha}^{\prime}$ for all $\alpha \geq \alpha_{1}$.

The quotient $T_{\alpha}:=U_{\alpha}^{\prime} / R_{\alpha}^{\prime}$ makes sense as a quasi-compact algebraic space locally of finitely presentation over $U_{\alpha} / R_{\alpha}=S_{\alpha}$ for $\alpha \geq \alpha_{1}$, and it is quasi-separated since $R_{\alpha}^{\prime} \rightarrow U_{\alpha}^{\prime} \times_{S_{\alpha}} U_{\alpha}^{\prime}$ is quasi-compact and $S_{\alpha}$ is quasi-separated. In particular, $\left\{T_{\alpha}\right\}$ is an inverse system of qcqs algebraic spaces. We clearly have $S_{\alpha^{-}}$ isomorphisms $T_{\alpha} \simeq T_{\alpha_{1}} \times_{S_{\alpha_{1}}} S_{\alpha}$ compatibly with change in $\alpha \geq \alpha_{1}$, so $\left\{T_{\alpha}\right\}$ has affine transition maps and there is an $S$-isomorphism $T \simeq T_{\alpha_{1}} \times_{S_{\alpha_{1}}} S$. In particular, $T$ is identified with $\lim _{\alpha} T_{\alpha}$ over $S$.

But we are considering the case when $T$ is a scheme, so by Lemma A.3.3 it follows that $T_{\alpha}$ is a scheme for sufficiently large $\alpha$ provided that some $T_{\alpha^{\prime}}$ is affine over an algebraic space of finite presentation over $\mathbf{Z}$. For example, we have have solved the case when $T$ is a scheme (with the stronger conclusion that we can choose the $T_{\alpha}$ to be schemes) provided that the $S_{\alpha}$ are of finite presentation over $\mathbf{Z}$, as then all $T_{\alpha}$ above are of finite presentation over $\mathbf{Z}$ as well (so Lemma A.3.3 is applicable to the inverse system $\left\{T_{\alpha}\right\}$ ). 
Now suppose more generally that $T$ is an algebraic space, but assume that all $S_{\alpha}$ are of finite presentation over $\mathbf{Z}$. (The reason for this temporary extra assumption is that the case when $T$ is a scheme has thus far been settled only in such cases, though with the stronger conclusion that the $T_{\alpha}$ can be chosen to be schemes in such cases.) Fix a qcqs étale cover $U^{\prime} \rightarrow T$ by a scheme, so $R^{\prime}=U^{\prime} \times_{T} U^{\prime}$ is also a scheme and both $U^{\prime}$ and $R^{\prime}$ are qcqs over $S=\lim S_{\alpha}$. We may apply to $U^{\prime}$ and $R^{\prime}$ the settled case of schemes (with all $S_{\alpha}$ of finite presentation over $\mathbf{Z}$ ), so by restricting to sufficiently large $\alpha$ we can construct cartesian limit presentations $U^{\prime}=\lim _{\alpha} U_{\alpha}^{\prime}$ and $R^{\prime}=\lim _{\alpha} R_{\alpha}^{\prime}$ in finitely presented schemes (with affine transition maps) over the $S_{\alpha}$ 's.

By Corollary A.3.2, the $S$-map $\delta^{\prime}: R^{\prime} \rightarrow U^{\prime} \times{ }_{S} U^{\prime}$ arises from a compatible system of $S_{\alpha}$-maps $\delta_{\alpha}^{\prime}: R_{\alpha}^{\prime} \rightarrow$ $U_{\alpha}^{\prime} \times S_{\alpha} U_{\alpha}^{\prime}$ for large $\alpha$. For fixed $i \in\{1,2\}$, the system of $S_{\alpha}$-maps $p_{i, \alpha}^{\prime}: R_{\alpha}^{\prime} \rightarrow U_{\alpha}^{\prime}$ between finitely presented $S_{\alpha}$-schemes is cartesian with respect to change in $\alpha$ and has limit $p_{i}^{\prime}: R^{\prime} \rightarrow U^{\prime}$ that is étale. Exactly as we have just argued above in our treatment of the case when $T$ is a scheme, by restricting to large $\alpha$ we can arrange that each $p_{i, \alpha}^{\prime}$ is étale and that each $\delta_{\alpha}^{\prime}$ is a monomorphism. A further application of the same method handles the symmetry and transitivity aspects, so $R_{\alpha}^{\prime} \rightrightarrows U_{\alpha}^{\prime}$ is a cartesian inverse system of étale equivalence relations in finitely presented schemes over $S_{\alpha}$ for large $\alpha$.

The quotients $T_{\alpha}=U_{\alpha}^{\prime} / R_{\alpha}^{\prime}$ form a cartesian inverse system of finitely presented algebraic spaces over $S_{\alpha}$, so $\left\{T_{\alpha}\right\}$ has affine transition maps. The limit $T^{\prime}:=\lim T_{\alpha}$ therefore makes sense as an algebraic space over $S$ and for a large $\alpha_{0}$ we have an $S$-isomorphism

$$
T^{\prime} \simeq T_{\alpha_{0}} \times_{S_{\alpha_{0}}} S=\left(U_{\alpha_{0}}^{\prime} / R_{\alpha_{0}}^{\prime}\right) \times_{S_{\alpha_{0}}} S \simeq U^{\prime} / R^{\prime}=T
$$

Thus, we have solved the general problem when the $S_{\alpha}$ are all of finite presentation over $\mathbf{Z}$, including the refined claim that (in such cases) when $T$ is a scheme we may choose the $T_{\alpha}$ to all be schemes.

Finally, we consider the general case by relaxing the assumption that all $S_{\alpha}$ are of finite presentation over $\mathbf{Z}$ to the assumption that some $S_{\alpha^{\prime}}$ is affine over an algebraic space $\mathscr{S}$ of finite presentation over $\mathbf{Z}$. We only consider $\alpha \geq \alpha^{\prime}$, so all $S_{\alpha}$ are affine over $\mathscr{S}$. Arguing via [K, III, Thm. 1.1, Cor. 1.2] as in the proof of Lemma A.3.3, we get another limit presentation $S=\lim _{\longleftarrow} S_{\gamma}^{\prime}$ with $\left\{S_{\gamma}^{\prime}\right\}$ an inverse system with affine transition maps of algebraic spaces over $\mathscr{S}$ with each map $S_{\gamma}^{\prime} \rightarrow \mathscr{S}$ affine and finitely presented. Thus, all $S_{\gamma}^{\prime}$ are finitely presented over $\mathbf{Z}$, so the settled cases imply that the finitely presented map $T \rightarrow S$ descends to a finitely presented map $T_{0} \rightarrow S_{\gamma_{0}}^{\prime}$ for some $\gamma_{0}$, and that we can choose $T_{0}$ to be a scheme when $T$ is a scheme.

Apply Proposition A.3.1 to the finitely presented map $S_{\gamma_{0}}^{\prime} \rightarrow \mathscr{S}$ and the inverse system $\left\{S_{\alpha}\right\}$ over $\mathscr{S}$ with limit $S$. This gives that the canonical $\mathscr{S}$-map $S=\lim _{S_{\alpha}} \rightarrow S_{\gamma_{0}}^{\prime}$ factors through an $\mathscr{S}$-map $S_{\alpha_{0}} \rightarrow S_{\gamma_{0}}^{\prime}$ for some $\alpha_{0}$. Note that this map is affine, since $S_{\alpha_{0}}$ and $S_{\gamma_{0}}^{\prime}$ are affine over $\mathscr{S}$. Define $T_{\alpha_{0}}=T_{0} \times S_{\gamma_{0}}^{\prime} S_{\alpha_{0}}$ and

$$
T_{\alpha}:=T_{\alpha_{0}} \times_{S_{\alpha_{0}}} S_{\alpha}=T_{0} \times_{S_{\gamma_{0}}^{\prime}} S_{\alpha}
$$

for all $\alpha \geq \alpha_{0}$. Then $\left\{T_{\alpha}\right\}$ is an inverse system with affine transition maps of finitely presented objects over $\left\{S_{\alpha}\right\}$ and

$$
T_{\alpha_{0}} \times S_{\alpha_{0}} S=T_{0} \times_{S_{\gamma_{0}}^{\prime}} S=T
$$

over $S$. Also, if $T$ is a scheme then we can choose $T_{0}$ to be a scheme, so then every $T_{\alpha}$ is a scheme since it is affine over $T_{0}$. Thus, we are done.

Corollary A.3.5. In the setup of Corollary A.3.2, let $f_{\alpha}: X_{\alpha} \rightarrow Y_{\alpha}$ over $S_{\alpha}$ be a compatible system maps inducing $f: X \rightarrow Y$ over $S$ in the limit. Assume that some $S_{\alpha^{\prime}}$ is affine over an algebraic space of finite presentation over $\mathbf{Z}$.

The map $f$ satisfies $\mathbf{P}$ if and only if $f_{\alpha}$ does for sufficiently large $\alpha$, where $\mathbf{P}$ is any of the properties of morphisms as in [EGA, $\mathrm{IV}_{3}, 8.10 .5(\mathrm{i})$-(xii), 11.2.6(ii); $\left.\mathrm{IV}_{4}, 17.7 .8(\mathrm{ii})\right]$.

The hypothesis that some $S_{\alpha^{\prime}}$ is affine over an algebraic space of finite presentation over $\mathbf{Z}$ is needed solely because of its role in the previous two results (which are used in the following proof), so as with those results we can eliminate this hypothesis once absolute noetherian approximation is proved for qcqs algebraic spaces in 3.1. The proof of this approximation result uses Corollary A.3.5 only in cases with all $S_{\alpha}$ of finite presentation over $\mathbf{Z}$, so there is no circularity. 
Proof. The descent of properties of morphisms which are étale-local on the source and target does not require the hypothesis on some $S_{\alpha^{\prime}}$ and is easily reduced to the known case of schemes treated in the given references in [EGA, IV]. The remaining properties (all in [EGA, $\left.\mathrm{IV}_{3}, 8.10 .5\right]$ ) are étale-local on the base, so we may assume that all $S_{\alpha}, S, Y_{\alpha}$, and $Y$ are affine schemes. The key point is that if $f$ is representable in schemes (i.e., if $X$ is a scheme) then so is $f_{\alpha}$ for large $\alpha$ (i.e., $X_{\alpha}$ is a scheme for large $\alpha$ ), as follows from Lemma A.3.3. This reduces everything to the known case of schemes except for the properties of being surjective, radiciel, quasi-finite, or proper. The first three are fibral properties cutting out a constructible locus in the base, and so the proofs for these conditions in the scheme case carry over to the case of algebraic spaces. Indeed, constructible loci in qcqs algebraic spaces interact with limits exactly as in the scheme case [EGA, $\left.\mathrm{IV}_{3}, 8.3 .4\right]$ (as one shows by working étale-locally to reduce to the case of schemes), so the behavior of fibral properties with respect to limits as in [EGA, $\mathrm{IV}_{3}, 9.3 .3$ ] holds for algebraic spaces too.

As for properness, if $f$ is proper then the finitely presented map $f_{\alpha}: X_{\alpha} \rightarrow Y_{\alpha}$ is at least separated for large $\alpha$. Fixing such an $\alpha_{0}$, since $Y_{\alpha_{0}}$ is affine we can express $Y_{\alpha_{0}}$ as the limit of an inverse system of affine schemes of finite type over $\mathbf{Z}$ and then use Proposition A.3.4 and the settled descent of separatedness to descend $f_{\alpha_{0}}$ to a finitely presented and separated map $X_{0} \rightarrow Y_{0}$ from an algebraic space to a finite type Z-scheme. By Chow's Lemma for separated maps of finite type between noetherian algebraic spaces, there is a surjective, proper, and finitely presented map $P_{\alpha_{0}} \rightarrow X_{\alpha_{0}}$ with $P_{\alpha_{0}}$ a scheme. The induced $S_{\alpha}$-maps $P_{\alpha} \rightarrow Y_{\alpha}$ for $\alpha \geq \alpha_{0}$ only involve schemes, and the limit map $P \rightarrow Y$ over $S$ is proper since $f: X \rightarrow Y$ is proper. Hence, by the known scheme case $P_{\alpha}$ is $Y_{\alpha}$-proper for large $\alpha$, so surjectivity of the $Y_{\alpha}$-map $P_{\alpha} \rightarrow X_{\alpha}$ forces the separated and finite type map $X_{\alpha} \rightarrow Y_{\alpha}$ to be proper for large $\alpha$.

We end this section by recording a result on how inverse limit presentations of quasi-compact open subschemes can be extended to such presentations of an ambient qcqs scheme at the expense of passing to a cofinal subsystem of the given inverse system.

Lemma A.3.6. Let $X$ be a qcqs scheme, and $V \subseteq X$ a quasi-compact open subscheme. Assume that $X$ is endowed with a structure of $\Lambda$-scheme for a noetherian ring $\Lambda$ (e.g., $\Lambda=\mathbf{Z})$, and choose an inverse system $\left\{V_{i}\right\}_{i \in I}$ of finite type $\Lambda$-schemes with affine and schematically dominant transition maps such that $V \simeq \lim _{i} V_{i}$.

This can be extended to a limit presentation of $X$ in the sense that there is an inverse system $\left\{X_{j}\right\}_{j \in J}$ of finite type $\Lambda$-schemes with affine and schematically dominant transition maps, a cofinal map of directed sets $\psi: J \rightarrow I$, and a compatible system of open immersions $V_{\psi(j)} \hookrightarrow X_{j}$ over $\Lambda$ such that the diagrams

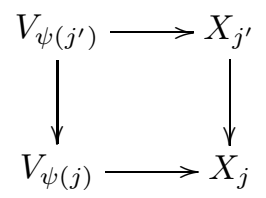

are cartesian for all $j^{\prime} \geq j$ and the open immersion $V=\lim _{\longleftarrow} V_{i} \simeq \lim _{\longleftarrow} V_{\psi(j)} \hookrightarrow \lim _{X_{j}}$ extends to an isomorphism $X \simeq \lim _{\longleftarrow} X_{j}$.

Such an inverse system $\left\{V_{i}\right\}$ always exists for any $V \subseteq X$, by [TT, Thm. C.9] and an argument with scheme-theoretic images as in the proof of Lemma 3.1.3.

Proof. Choose a non-empty finite collection of affine open subschemes $\left\{U_{1}, \ldots, U_{m}\right\}$ of $X$ such that $V$ together with the $U_{k}$ cover $X$. (For example, we could take $\left\{U_{k}\right\}$ to be an affine open covering of $X$.) We shall argue by induction on $m$. The case $m=1$ is established in the proof of [TT, Thm. C.9], and here it is used that $\Lambda$ is noetherian and the affine transition maps in $\left\{V_{i}\right\}$ are schematically dominant. In general, if $m>1$ then we may apply induction to $V^{\prime}=V \cup\left(U_{1} \cup \cdots \cup U_{m-1}\right)$ in the role of $X$ and then apply the case $m=1$ to the inclusion of $V^{\prime}$ into $X$ (with $X=V^{\prime} \cup U_{m}$ ).

A.4. Square-zero thickenings of algebraic spaces. In order to reduce the proof of Nagata's theorem to the reduced case, we need to relate square-zero thickenings of an algebraic space $X$ to (suitable) square-zero 
extensions of the sheaf of rings $\mathscr{O}_{X}$ on $X_{\text {ét }}$. It is convenient to express this in terms of an equivalence of categories, as follows.

Let $\mathscr{C}^{\prime}$ denote the category of pairs $\left(X^{\prime}, \mathscr{I}\right)$ consisting of an algebraic space $X^{\prime}$ and a quasi-coherent sheaf of ideals $\mathscr{I} \subseteq \mathscr{O}_{X^{\prime}}$ on $X_{\text {ét }}^{\prime}$ such that $\mathscr{I}^{2}=0$. Let $\mathscr{C}$ denote the category of pairs $\left(X, \mathscr{A} \rightarrow \mathscr{O}_{X}\right)$ consisting of an algebraic space $X$ and a surjective map $\theta: \mathscr{A} \rightarrow \mathscr{O}_{X}$ of sheaves of rings on $X_{\text {ét }}$ whose kernel $\mathscr{J} \subseteq \mathscr{A}$ is a square-zero ideal that is quasi-coherent as an $\mathscr{O}_{X}$-module.

Fix an object $\left(X^{\prime}, \mathscr{I}\right)$ in $\mathscr{C}^{\prime}$. Let $X$ denote the closed subspace of $X^{\prime}$ cut out by $\mathscr{I}$. Pullback along $X_{\text {ét }} \rightarrow X_{\text {ét }}^{\prime}$ induces an equivalence of topoi, by Proposition A.1.3, so for a sheaf of sets $\mathscr{F}^{\prime}$ on $X_{\text {ét }}^{\prime}$ we will therefore abuse notation (when the context makes it clear) by also writing $\mathscr{F}^{\prime}$ to denote the pullback sheaf on $X_{\text {ét }}$. For example, we view $\mathscr{O}_{X^{\prime}}$ as a sheaf of rings on $X_{\text {ét }}$ in this way, and $\mathscr{I}$ as a square-zero sheaf of ideals in $\mathscr{O}_{X^{\prime}}$ on $X_{\text {ét }}$. There is an evident quotient map $\mathscr{O}_{X^{\prime}} \rightarrow \mathscr{O}_{X}$ of sheaves on $X_{\text {ét }}$ with kernel $\mathscr{I}$ whose induced module structure over $\mathscr{O}_{X^{\prime}} / \mathscr{I} \simeq \mathscr{O}_{X}$ on $X_{\text {ét }}$ is clearly quasi-coherent. Thus, $\left(X^{\prime}, \mathscr{I}\right) \rightsquigarrow\left(X, \mathscr{O}_{X^{\prime}} \rightarrow \mathscr{O}_{X}\right)$ is a functor from $\mathscr{C}^{\prime}$ to $\mathscr{C}$.

Theorem A.4.1. The above functor $\mathscr{C}^{\prime} \rightarrow \mathscr{C}$ is an equivalence of categories.

This theorem says that for any algebraic space $X$, to give a square-zero thickening of $X$ as an algebraic space is functorially the same as to choose a quasi-coherent sheaf $\mathscr{J}$ on $X_{\text {ét }}$ and a sheaf of rings $\mathscr{A}$ on $X_{\text {ét }}$ that is a square-zero extension of $\mathscr{O}_{X}$ by the $\mathscr{O}_{X}$-module $\mathscr{J}$. This latter point of view is expressed entirely in terms of the étale topos of $X$ and so is well-suited to deformation-theoretic considerations (such as with the cotangent complex).

Remark A.4.2. Although we state and prove Theorem A.4.1 without noetherian hypotheses, it is only used in this paper the noetherian case (in 92.1 ). Aside from using a couple of self-contained results (Lemma 3.1.4 and Proposition A.1.3), our proof uses Corollary 3.1.12, which rests on Theorem 1.2.2, whose proof in turn relies on almost everything in $\$ 3.1$ and $\$$ A.3. However, the noetherian case of Corollary 3.1 .12 is an old result of Knutson [K, III, Thm. 3.3]. Consequently, the reader only interested in 92 may safely restrict attention to the simpler noetherian case with quasi-coherence replaced with coherence in several places (since a square-zero extension of a noetherian ring $A_{0}$ by a finite $A_{0}$-module is automatically a noetherian ring).

Proof of Theorem A.4.1. Step 1. (Faithfulness) For objects $\left(X_{1}^{\prime}, \mathscr{I}_{1}\right)$ and $\left(X_{2}^{\prime}, \mathscr{I}_{2}\right)$ in $\mathscr{C}^{\prime}$ consider two maps $f^{\prime}, h^{\prime}: X_{1}^{\prime} \rightrightarrows X_{2}^{\prime}$ such that (i) $f^{\prime}$ and $h^{\prime}$ carry $X_{1}$ into $X_{2}$, (ii) the induced maps $f, h: X_{1} \rightrightarrows X_{2}$ coincide, and (iii) the resulting pairs of maps

$$
\mathscr{O}_{X_{2}^{\prime}} \rightrightarrows f_{*}\left(\mathscr{O}_{X_{1}^{\prime}}\right)=h_{*}\left(\mathscr{O}_{X_{2}^{\prime}}\right)
$$

of sheaves on $\left(X_{2}\right)_{\text {ét }}$ coincide. We wish to show that $f^{\prime}=h^{\prime}$. In case $X_{1}^{\prime}$ and $X_{2}^{\prime}$ are schemes, so morphisms between them can be considered within the category of ringed spaces (rather than ringed topoi), the desired equality is obvious. In general we will use étale covers by schemes to reduce the faithfulness problem to the settled scheme case.

Pick an étale covering $\pi_{2}^{\prime}: U_{2}^{\prime} \rightarrow X_{2}^{\prime}$ by a scheme, and let $\pi_{2}: U_{2} \rightarrow X_{2}$ denote the pullback étale scheme covering. The resulting pullback étale covers

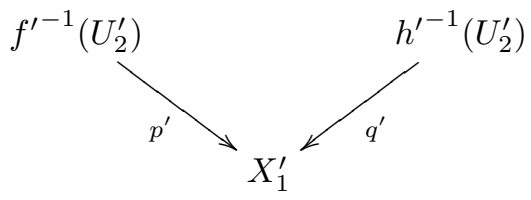

restrict over $X_{1} \subseteq X_{1}^{\prime}$ to algebraic space étale covers $f^{-1}\left(U_{2}\right)$ and $h^{-1}\left(U_{2}\right)$ of $X_{1}$ that are naturally identified since $f=h$. This $X_{1}$-isomorphism $\phi:\left.\left.f^{\prime-1}\left(U_{2}^{\prime}\right)\right|_{X_{1}} \simeq h^{\prime-1}\left(U_{2}^{\prime}\right)\right|_{X_{1}}$ between algebraic spaces étale over $X_{1}$ uniquely lifts to an $X_{1}^{\prime}$-isomorphism $\phi^{\prime}: f^{\prime-1}\left(U_{2}^{\prime}\right) \simeq h^{\prime-1}\left(U_{2}^{\prime}\right)$ between algebraic spaces étale over $X_{1}^{\prime}$, due to the topological invariance of the étale site of algebraic spaces (Proposition A.1.3). Since $p^{\prime}$ and $q^{\prime}$ are étale 
surjections and $q^{\prime} \circ \phi^{\prime}=p^{\prime}$, to prove the equality $f^{\prime}=h^{\prime}$ it suffices to prove that the diagram

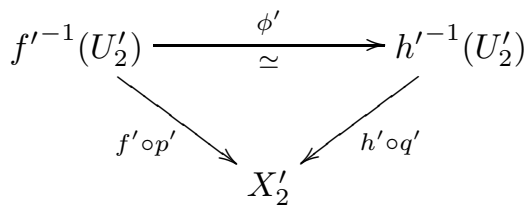

commutes.

Choose an étale scheme cover $\psi^{\prime}: U_{1}^{\prime} \rightarrow f^{\prime-1}\left(U_{2}^{\prime}\right)$ and define the étale scheme covering map $U_{1}^{\prime} \rightarrow h^{\prime-1}\left(U_{2}^{\prime}\right)$ to be $\phi^{\prime} \circ \psi^{\prime}$, so these define a common étale covering map $\pi_{1}^{\prime}: U_{1}^{\prime} \rightarrow X_{1}^{\prime}$ since $\phi^{\prime}$ is an $X_{1}^{\prime}$-isomorphism. Composing $f^{\prime} \circ p^{\prime}$ and $h^{\prime} \circ q^{\prime}$ back to $U_{1}^{\prime}$ via $\psi^{\prime}$ and $\phi^{\prime} \circ \psi^{\prime}$ respectively recovers the pair of maps $f^{\prime} \circ \pi_{1}^{\prime}, h^{\prime} \circ \pi_{1}^{\prime}$ : $U_{1}^{\prime} \rightrightarrows X_{2}^{\prime}$, so we get a co-commutative diagram

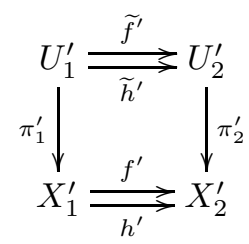

in which $\widetilde{f}^{\prime}$ is $U_{1}^{\prime} \stackrel{\psi^{\prime}}{\rightarrow} f^{\prime-1}\left(U_{2}^{\prime}\right) \rightarrow U_{2}^{\prime}$ and $\widetilde{h}^{\prime}$ is $U_{1}^{\prime} \stackrel{\phi^{\prime} \circ \psi^{\prime}}{\longrightarrow} h^{\prime-1}\left(U_{2}^{\prime}\right) \rightarrow U_{2}^{\prime}$. But the pair $\left(\widetilde{f}^{\prime}, \widetilde{h}^{\prime}\right)$ satisfies the same initial hypotheses as the pair $\left(f^{\prime}, h^{\prime}\right)$, using the quasi-coherent square-zero ideals given by the pullbacks of $\mathscr{I}_{1}^{\prime}$ to $U_{1}^{\prime}$ and $\mathscr{I}_{2}^{\prime}$ to $U_{2}^{\prime}$. Hence, by the settled scheme case we conclude that $\widetilde{f}^{\prime}=\widetilde{h}^{\prime}$, so $f^{\prime}=h^{\prime}$.

Step 2. (Fullness: reduction to schemes) For a pair of objects $\left(X_{1}^{\prime}, \mathscr{I}_{1}\right)$ and $\left(X_{2}^{\prime}, \mathscr{I}_{2}\right)$ in $\mathscr{C}^{\prime}$, suppose we are given a map $f: X_{1} \rightarrow X_{2}$ of algebraic spaces and a map $\theta^{\prime}: \mathscr{O}_{X_{2}^{\prime}} \rightarrow f_{*}\left(\mathscr{O}_{X_{1}^{\prime}}\right)$ of sheaves of rings on $\left(X_{2}\right)_{\text {ét }}$ (using the equivalences $\left(X_{1}\right)_{\text {ét }}=\left(X_{1}^{\prime}\right)_{\text {ét }}$ and $\left(X_{2}\right)_{\text {ét }}=\left(X_{2}^{\prime}\right)_{\text {ét }}$ to make sense of $\left.f_{*}\left(\mathscr{O}_{X_{1}^{\prime}}\right)\right)$ such that the diagram

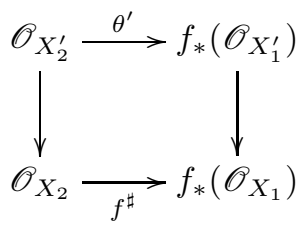

of sheaves on $\left(X_{2}\right)_{\text {ét }}$ commutes. We seek to construct a map $f^{\prime}: X_{1}^{\prime} \rightarrow X_{2}^{\prime}$ inducing the pair $\left(f, \theta^{\prime}\right)$.

By Step 1 such an $f^{\prime}$ is unique if it exists, so by descent we can compose along an étale scheme covering of $X_{1}^{\prime}$ to reduce to the case when $X_{1}^{\prime}$ (and hence $X_{1}$ ) is a scheme. We will reduce the proof of existence of $f^{\prime}$ to the case when $X_{2}^{\prime}$ is also a scheme. In the case that $X_{2}^{\prime}$ is a scheme there is trivially such an $f^{\prime}$ which uniquely solves our problem for the Zariski topology since we can then work with locally ringed spaces, but even in this scheme case there is more to do: we have to show that the scheme morphism which is a solution for the Zariski topology is a solution for the étale topology. Granting this additional property in the scheme case for now, let us see how to solve the general case.

Choose an étale scheme cover $\pi_{2}^{\prime}: U_{2}^{\prime} \rightarrow X_{2}^{\prime}$ and form the cartesian square

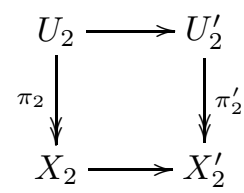

in which the horizontal arrows are closed immersions defined by square-zero ideals. In particular, the left side is an étale scheme covering. Pulling back $\pi_{2}$ along $f: X_{1} \rightarrow X_{2}$ defines an étale cover

$$
\pi_{1}: U_{1}:=X_{1} \times_{X_{2}} U_{2} \rightarrow X_{1}
$$


with $U_{1}$ a scheme since $X_{1}$ and $U_{2}$ are schemes, and by topological invariance of the étale site of algebraic spaces (Proposition A.1.3) this fits into a unique cartesian square of algebraic spaces

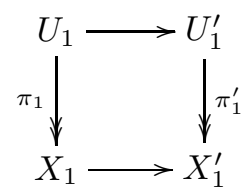

such that $\pi_{1}^{\prime}$ is étale (and necessarily surjective, since $\pi_{1}$ is). The underlying reduced space $\left(U_{1}^{\prime}\right)_{\text {red }}=\left(U_{1}\right)_{\text {red }}$ is a scheme, so by [K, III, Thm. 3.3] in the noetherian case and by Corollary 3.1 .12 in the general case we conclude that $U_{1}^{\prime}$ is actually a scheme. (See Remark A.4.2.)

The natural map $\tilde{f}=\pi_{2}^{*}(f): U_{1}=X_{1} \times_{X_{2}} U_{2} \rightarrow U_{2}$ fits into the top row of the diagram of schemes

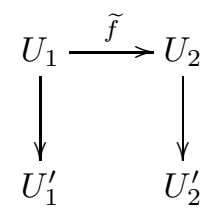

in which the vertical maps are closed immersions defined by the square-zero pullbacks of $\mathscr{I}_{1}$ and $\mathscr{I}_{2}$ along $\pi_{1}^{\prime}: U_{1}^{\prime} \rightarrow X_{1}^{\prime}$ and $\pi_{2}^{\prime}: U_{2}^{\prime} \rightarrow X_{2}^{\prime}$ respectively. The diagram (A.4.2.1) of sheaves of rings on $\left(X_{2}\right)$ ét restricts over the subcategory $\left(U_{2}\right)_{\text {ét }}$ to give a commutative diagram

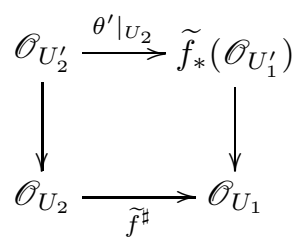

in which the vertical maps are the natural surjections. Hence, the pair $\left(\tilde{f},\left.\theta^{\prime}\right|_{U_{2}}\right)$ is a morphism

$$
\left(U_{1}, \mathscr{O}_{U_{2}^{\prime}} \rightarrow \mathscr{O}_{U_{1}}\right) \rightarrow\left(U_{2}, \mathscr{O}_{U_{2}^{\prime}} \rightarrow \mathscr{O}_{U_{2}}\right)
$$

in the category $\mathscr{C}$. Since we are assuming that the fullness problem is solved for schemes (with the étale topology), the morphism (A.4.2.4) arises from a unique morphism $\tilde{f}^{\prime}: U_{1}^{\prime} \rightarrow U_{2}^{\prime}$ of algebraic spaces fitting into the bottom side of A.4.2.2 as a commutative diagram and compatible with $\left.\theta^{\prime}\right|_{U_{2}}$.

Consider the resulting diagram

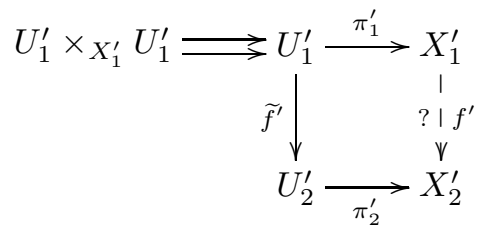

Composing the map $\pi_{2}^{\prime} \circ \tilde{f}^{\prime}: U_{1}^{\prime} \rightarrow X_{2}^{\prime}$ with the projections $U_{1}^{\prime} \times_{X_{1}^{\prime}} U_{1}^{\prime} \rightrightarrows U_{1}^{\prime}$ gives a pair of maps $U_{1}^{\prime} \times_{X_{1}^{\prime}} U_{1}^{\prime} \rightrightarrows$ $X_{2}^{\prime}$ that coincide, due to Step 1 (using the square-zero quasi-coherent pullback of $\mathscr{I}_{1}$ on $U_{1}^{\prime} \times_{X_{1}^{\prime}} U_{1}^{\prime}$ and the commutativity of (A.4.2.1 and (A.4.2.3), so by descent we can uniquely fill in the arrow $f^{\prime}: X_{1}^{\prime} \rightarrow X_{2}^{\prime}$ in A.4.2.5 to make a commutative square. It is easy to check (with the help of $\tilde{f}^{\prime}$ ) that $f^{\prime}$ is the morphism from $\left(X_{1}^{\prime}, \mathscr{I}_{1}\right)$ to $\left(X_{2}^{\prime}, \mathscr{I}_{2}\right)$ in $\mathscr{C}^{\prime}$ that we sought to construct. This completes the reduction of the fullness problem to the special case of schemes (with the étale topology!). We will address this in Step 3.

Step 3. (The case of schemes) We now prove that the faithful functor $\mathscr{C}^{\prime} \rightarrow \mathscr{C}$ is full on the full subcategory of pairs $\left(X^{\prime}, \mathscr{I}\right)$ for which $X^{\prime}$ is a scheme (with the étale topology) and that its essential image contains all pairs $\left(X, \mathscr{A} \rightarrow \mathscr{O}_{X}\right)$ for which $X$ is a scheme (with the étale topology). The fullness for schemes is trivial to check for the Zariski topology using the viewpoint of locally ringed spaces. Fullness for schemes 
with the étale topology then follows formally from two facts: (i) for a scheme $X^{\prime}$, the stalks of $\mathscr{O}_{X_{\text {ét }}^{\prime}}$ at geometric points of $X_{\text {ét }}^{\prime}$ are the strict henselizations of the Zariski local rings of $\mathscr{O}_{X^{\prime}}$, and (ii) the uniqueness aspect of the universal mapping property for the strict henselization of a local ring (relative to a specified separable closure of its residue field). By Step 2, fullness has now been proved in general.

Now consider a pair $\left(X, \mathscr{A} \rightarrow \mathscr{O}_{X}\right)$ for a scheme $X$ with the étale topology. We seek to construct a pair $\left(X^{\prime}, \mathscr{I}\right)$ in $\mathscr{C}^{\prime}$ giving rise to this, and will do so with $X^{\prime}$ a scheme. By the settled full faithfulness, it suffices to solve this problem Zariski-locally on $X$, so we may assume $X=\operatorname{Spec} A$ is an affine scheme. We then let $A^{\prime}=\mathscr{A}(X)$, so $A^{\prime} \rightarrow A$ is surjective since $X$ has vanishing higher Zariski cohomology for quasi-coherent $\mathscr{O}_{X}$-modules. The kernel $I=\operatorname{ker}\left(A^{\prime} \rightarrow A\right)$ is a square-zero ideal in $A^{\prime}$, and so ( $\left.\operatorname{Spec} A^{\prime}, \widetilde{I}\right)$ with the étale topology is the natural candidate to consider for $\left(X^{\prime}, \mathscr{I}\right)$. Consideration of Zariski stalks shows that that the natural map $\left.\mathscr{O}_{\mathrm{Spec}} A^{\prime} \rightarrow \mathscr{A}\right|_{X_{\mathrm{Zar}}}$ is an isomorphism. Our problem is to show that this (necessarily uniquely) lifts to an isomorphism $\mathscr{O}_{\mathrm{Spec} A^{\prime} \text {,ét }} \simeq \mathscr{A}$ of sheaves of rings on $X_{\text {ét }}$ respecting the identification $\mathscr{O}_{\mathrm{Spec} A}=\mathscr{O}_{X}$ of quotient rings on $X_{\text {ét }}$ and the identification of ideal sheaves $\widetilde{I}_{\text {ét }} \simeq \mathscr{I}:=\operatorname{ker}\left(\mathscr{A} \rightarrow \mathscr{O}_{X}\right)$ on $X_{\text {ét }}$.

Let $X^{\prime}=\operatorname{Spec} A^{\prime}$. Consider geometric points $\bar{x}$ of $X_{\text {ét }}^{\prime}=X_{\text {ét }}$ that are algebraic over their physical image points $x \in X$. Observe that $\mathscr{A}_{\bar{x}}$ is a strictly henselian local ring with residue field $k(\bar{x})$ since its quotient $\mathscr{O}_{X_{\text {ét }}}$ by a square-zero ideal sheaf has strictly henselian local $\bar{x}$-stalk with residue field $k(\bar{x})$. Letting $\pi: X_{\text {ét }} \rightarrow X_{\text {Zar }}$ be the natural map of sites, the given isomorphism $\mathscr{O}_{X_{\mathrm{Zar}}^{\prime}} \simeq \pi_{*} \mathscr{A}$ corresponds to a map $\pi^{-1} \mathscr{O}_{X_{\mathrm{Zar}}^{\prime}} \rightarrow \mathscr{A}_{\text {on }}$ $X_{\text {ét }}$ that on $\bar{x}$-stalks is the natural local map $\theta_{\bar{x}}: \mathscr{O}_{X^{\prime}, x} \rightarrow \mathscr{A}_{\bar{x}}$ inducing $k(x) \rightarrow k(\bar{x})$ on residue fields. But the map on $\bar{x}$-stalks induced by the natural map $\pi^{-1} \mathscr{O}_{X_{\text {zar }}^{\prime}} \rightarrow \mathscr{O}_{X_{\text {ét }}^{\prime}}$ is identified with the unique local map $\mathscr{O}_{X^{\prime}, x} \rightarrow \mathscr{O}_{X^{\prime}, \bar{x}}^{\text {sh }}$ lifting $k(x) \rightarrow k(\bar{x})$ on residue fields, so by the universal property of strict henselization there is at most one way to fill in the dotted arrow in the commutative square

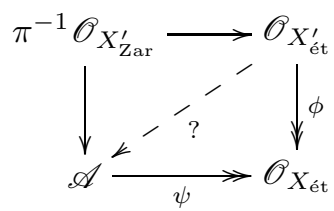

to make commutative triangles of sheaves of rings. (Such a diagonal map is necessarily local on stalks and an isomorphism on residue fields since $\phi$ and $\psi$ are quotients by square-zero ideal sheaves.) The lower triangle commutes if the upper one does, by the universal property of strict henselizations. Moreover, both ideal sheaves $\operatorname{ker} \phi$ and ker $\psi$ are quasi-coherent on $X_{\text {ét }}$, and a map between quasi-coherent sheaves for the étale topology of a scheme is an isomorphism if and only if it is an isomorphism for the Zariski topology. Thus, since a diagonal map as in (A.4.2.6) must be an isomorphism on $X_{\text {Zar }}^{\prime}=X_{\text {Zar }}$ if it exists (as the left and top sides are isomorphisms over $X_{\text {Zar }}^{\prime}$ ), it must induce an isomorphism between the quasi-coherent ker $\phi$ and ker $\psi$ on $X_{\text {ét }}$ and hence be an isomorphism if it exists.

We are now reduced to constructing a map of sheaves of $\pi^{-1} \mathscr{O}_{X_{\mathrm{Zar}}}$-algebras $\mathscr{O}_{X_{\text {ét }}^{\prime}} \rightarrow \mathscr{A}$ over $X_{\text {ét }}=X_{\text {ét. }}^{\prime}$. We will do this functorially on sections over étale maps $h: U \rightarrow X$ with $U$ an affine scheme. Via the equivalence of $X_{\text {ét }}$ and $X_{\text {ét }}^{\prime}$, there is a unique cartesian diagram of schemes

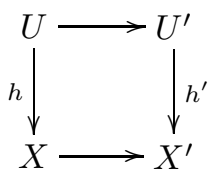

in which $h^{\prime}$ is étale, and $U^{\prime}$ is affine since the square-zero closed subscheme $U$ is affine. The natural map $\mathscr{A}(U) \rightarrow \mathscr{O}_{X_{\text {ét }}}(U)=\mathscr{O}_{U}(U)$ is surjective since $U$ is affine and $\mathscr{J}:=\operatorname{ker}\left(\mathscr{A} \rightarrow \mathscr{O}_{X}\right)$ is quasi-coherent on $X_{\text {ét }}$, so the natural map $i: U \rightarrow \operatorname{Spec} \mathscr{A}(U)$ is a closed immersion defined by the square-zero ideal $\mathscr{J}(U)$. 
By functoriality the diagram

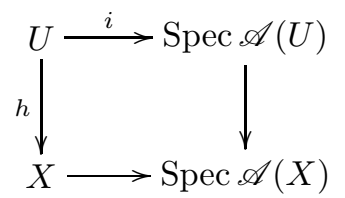

commutes, so the natural map $\operatorname{Spec} \mathscr{A}(U) \rightarrow \operatorname{Spec} \mathscr{A}(X)=: X^{\prime}$ has restriction to the square-zero closed subscheme $U$ (via $i$ ) that factors through the étale map $U^{\prime} \rightarrow X^{\prime}$ due to (A.4.2.7). By the functorial property of étale maps of schemes, there is a unique $X^{\prime}$-map $\operatorname{Spec} \mathscr{A}(U) \rightarrow U^{\prime}$ lifting the identity on the common square-zero closed subscheme $U$ over $X$. This provides an $A^{\prime}$-algebra map $\mathscr{O}_{X_{\text {ét }}^{\prime}}\left(U^{\prime}\right)=\mathscr{O}_{U^{\prime}}\left(U^{\prime}\right) \rightarrow \mathscr{A}(U)$, and by the uniqueness of this construction it is easily checked to be functorial in $U \rightarrow X$. Hence, we have constructed a $\pi^{-1}\left(\mathscr{O}_{X_{\text {Zar }}^{\prime}}\right)$-algebra map $\mathscr{O}_{X^{\prime}} \rightarrow \mathscr{A}$ over $X_{\text {ét }}=X_{\text {ét }}^{\prime}$, so we are done.

Step 4. (Essential surjectivity: general case) By Step 3, essential surjectivity is solved for objects $\left(X, \mathscr{A} \rightarrow \mathscr{O}_{X}\right)$ in $\mathscr{C}$ such that the algebraic space $\left(X, \mathscr{O}_{X}\right)$ is a scheme (with its étale topology), as well as full faithfulness in general. In particular, if an object $\left(X^{\prime}, \mathscr{I}\right)$ in $\mathscr{C}^{\prime}$ is carried to $\left(X, \mathscr{A}_{\rightarrow} \rightarrow \mathscr{O}_{X}\right)$ for a scheme $X$ then $X^{\prime}$ is a scheme by construction. We will now prove essential surjectivity in general.

Choose an étale scheme covering $U \rightarrow X$, and consider the object $\left(U,\left.\mathscr{A}\right|_{U_{\text {ét }}} \rightarrow \mathscr{O}_{U}\right)$ in $\mathscr{C}$; for ease of notation we will denote this as $\left(U,\left.\mathscr{A}\right|_{U_{\text {ét }}}\right)$. Since $U$ is a scheme, by essential surjectivity that we are assuming in the scheme case (with the étale topology) there is an object $\left(U^{\prime}, \mathscr{J}\right)$ in $\mathscr{C}^{\prime}$ that is carried to $\left(U,\left.\mathscr{A}\right|_{U_{\text {ét }}}\right)$, and $U^{\prime}$ is necessarily a scheme. Likewise, the fiber product $R:=U \times_{X} U$ is a scheme that is étale over $U$ in two ways and is étale over $X$ in a canonical way, so $\left(R,\left.\mathscr{A}\right|_{R_{\text {ét }}}\right)$ is an object in $\mathscr{C}$ with two natural maps to $\left(U,\left.\mathscr{A}\right|_{U_{\text {ét }}}\right)$. Thus, $\left(R,\left.\mathscr{A}\right|_{R_{\text {ét }}}\right)$ arises from a unique (up to unique isomorphism) object $\left(R^{\prime}, \mathscr{K}\right)$ in $\mathscr{C}^{\prime}$ with $R^{\prime}$ a scheme such that $R$ is identified with the zero scheme of $\mathscr{K}$ in $R^{\prime}$ and the equivalence $R_{\text {ét }}^{\prime} \simeq R_{\text {ét }}$

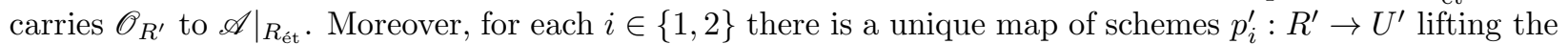
étale map $p_{i}: R \rightarrow U$ and identifying $\mathscr{O}_{U^{\prime}} \rightarrow p_{i *}^{\prime}\left(\mathscr{O}_{R^{\prime}}\right)$ on $U_{\text {ét }}$ with $\left.\mathscr{A}\right|_{U_{\text {ét }}} \rightarrow p_{i *}\left(\left.\mathscr{A}\right|_{R_{\text {ét }}}\right)$.

To prove that each $p_{i}^{\prime}$ is étale (and in particular locally of finite presentation) we give an alternative construction of $p_{i}^{\prime}$ as follows. The étale map $p_{i}: R \rightarrow U$ uniquely lifts to an étale map of schemes $q_{i}^{\prime}: R_{i}^{\prime} \rightarrow U^{\prime}$, so $\mathscr{O}_{R_{i}^{\prime}}$ viewed on $R_{\text {ét }}$ is identified with $\left.\mathscr{O}_{U^{\prime}}\right|_{R_{\text {ét }}}=\left.\mathscr{A}\right|_{R_{\text {ét }}}$. Hence, by the unique characterization of $\left(R^{\prime}, \mathscr{K}\right)$ we get unique $U^{\prime}$-isomorphisms $\left(R^{\prime}, p_{i}^{\prime}\right) \simeq\left(R_{i}^{\prime}, q_{i}^{\prime}\right)$. This shows that each $p_{i}^{\prime}$ is étale.

Since the diagram of schemes

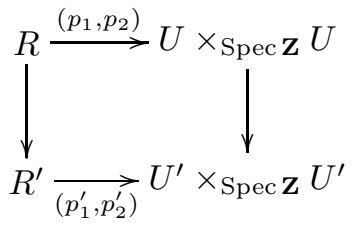

is cartesian (due to the constructions of $R^{\prime}$ and the $p_{i}^{\prime}$ ) and the top side is an equivalence relation, the easy full faithfulness for schemes with the Zariski topology can be used to construct a canonical groupoid structure on $R^{\prime} \rightarrow U^{\prime} \times U^{\prime}$ lifting the one on $R \rightarrow U \times U$. In other words, $R^{\prime} \rightrightarrows U^{\prime}$ is an étale equivalence relation in schemes, so the quotient $X^{\prime}=U^{\prime} / R^{\prime}$ makes sense as an algebraic space and Lemma 3.1.4 ensures that the commutative diagram

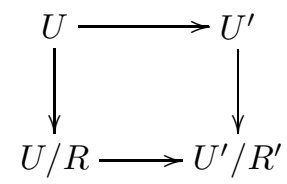

is cartesian. But the vertical maps are étale coverings and the top side is a square-zero thickening, so $X^{\prime}=U^{\prime} / R^{\prime}$ is a square-zero thickening of $X=U / R$. It is easy to check that this square-zero thickening $X^{\prime}$ of $X$ solves the original essential surjectivity problem for the object $\left(X, \mathscr{A} \rightarrow \mathscr{O}_{X}\right)$. 
A.5. Flattening for finite type maps. Our approximation arguments made essential use of results of Raynaud and Gruson from [RG, I, §5.7]. In this section, we wish to address a minor issue in the formulation of [RG, I, 5.7.10-5.7.14]: these results are stated for finite type maps $f: X \rightarrow S$ between qcqs algebraic spaces, but the proofs rest on a result [RG, I, 5.7.9] which is proved under finite presentation hypotheses.

This does not affect anything in the present paper, as we only use [RG, I, 5.7.10-5.7.14] in the proof of Lemma 2.2.6 where such results are applied to noetherian algebraic spaces, in which case the finite presentation requirement is satisfied. Hence, there is circularity in now using our approximation results to show that the results in [RG, I, 5.7.10-5.7.14] are true as stated with "finite type" rather than "finite presentation".

Once the two results [RG, I, 5.7.10, 5.7.11] are proved as stated in the finite type case, the arguments of Raynaud and Gruson for their subsequent results work as written. We explain below how to make the proofs of [RG, I, 5.7.10, 5.7.11] work in the finite type case by applying the preliminary result [RG, I, 5.7.9] (which has finite presentation hypotheses) to an auxiliary finitely presented map that is provided by Theorem 3.2.1

Lemma A.5.1. Let $h: V \rightarrow S$ be a flat and locally finitely presented map of algebraic spaces. If there exists a schematically dense open subspace $U \subseteq V$ with $\operatorname{dim}(U / S):=\sup _{s \in|S|} \operatorname{dim} U_{s} \leq n$ then $\operatorname{dim}(V / S) \leq n$.

Proof. By working étale-locally on $S$ and then on $V$, we may assume that $S$ and $V$ are affine schemes (so $h$ is finitely presented). It therefore suffices to show that if $S=\operatorname{Spec}(A)$ with $A$ a local ring then $\operatorname{dim}\left(V_{0}\right) \leq n$, where $V_{0}$ is the special fiber. Letting $\eta$ denote a generic point of $S$, by flatness and finite presentation for $h$ we have $\operatorname{dim}\left(V_{0}\right)=\operatorname{dim}\left(V_{\eta}\right)$. We can therefore replace $A$ with $A_{\eta}$ so that $A$ is a 0 -dimensional local ring. In this case, since $U$ is schematically dense in $V$ it is topologically dense, so $U_{\text {red }}$ is a topologically dense open subscheme of the scheme $V_{\text {red }}$ of finite type over the field $A_{\text {red }}$. Hence $\operatorname{dim}(V)=\operatorname{dim}\left(V_{\text {red }}\right)=\operatorname{dim}\left(U_{\text {red }}\right) \leq$ $n$.

Proposition A.5.2 ([ $\mathrm{RG}, \mathrm{I}, 5.7 .10])$. Let $f: X \rightarrow S$ be a finite type map of qcqs algebraic spaces, and let $U \subseteq S$ be a quasi-compact open subspace such that $f^{-1}(U) \rightarrow U$ has all fibers with dimension $\leq n$, with $n$ a fixed integer. Then there exists a $U$-admissible blow-up $g: S^{\prime} \rightarrow S$ such that the strict transform $X^{\prime} \rightarrow S^{\prime}$ of $f$ with respect to $g$ has all fibers with dimension $\leq n$.

Proof. The case of finitely presented $f$ is what is proved by Raynaud and Gruson, and we will use Theorem 3.2 .1 to make their method of proof work in the general finite type case.

By Theorem 3.2.1, we may choose a closed immersion $i: X \hookrightarrow \bar{X}$ over $S$ into an algebraic space $\bar{X}$ of finite presentation over $S$. Let $\bar{f}: \bar{X} \rightarrow S$ denote the structure map. By Chevalley's semi-continuity theorem for fiber dimension of locally finite type maps [EGA, $\mathrm{IV}_{3}, 13.1 .3$ ] (which works for algebraic spaces by using étale localization to reduce to the case of schemes), the locus

$$
W=\left\{\bar{x} \in \bar{X} \mid \operatorname{dim}_{\bar{x}}\left(\bar{f}^{-1}(\bar{f}(\bar{x}))\right) \leq n\right\}
$$

is open in $\bar{X}$.

By [RG, I, 5.7.8], the quasi-coherent ideal sheaf $\mathscr{I}$ of $X$ in $\bar{X}$ is the direct limit of its finite type quasicoherent $\mathscr{O}_{\bar{X}}$-submodules $\mathscr{I}_{\lambda}$. Hence, $X=\lim _{\longleftarrow} X_{\lambda}$ where $\left\{X_{\lambda}\right\}$ is the inverse system (with affine transition maps) of finitely presented closed subspaces of $\bar{X}$ that contain $X$. If $f_{\lambda}: X_{\lambda} \rightarrow S$ denotes the finitely presented structure map then $\left\{f_{\lambda}^{-1}(U)\right\}$ is an inverse system of finitely presented closed subspaces of $\bar{f}^{-1}(U)$ with $\lim _{\lambda} f_{\lambda}^{-1}(U)=f^{-1}(U)$. By hypothesis, the open subspace $W$ in the qcqs $\bar{X}$ contains $f^{-1}(U)$. Hence, if $Z$ denotes $\bar{X}-W$ with its reduced structure then $\left\{Z \cap f_{\lambda}^{-1}(U)\right\}$ is an inverse system of qcqs algebraic spaces with limit $Z \cap f^{-1}(U)=\emptyset$. It follows that $Z \cap f_{\lambda}^{-1}(U)$ is empty for sufficiently large $\lambda$, so $f_{\lambda}^{-1}(U) \subseteq W$ for sufficiently large $\lambda$. For any $\bar{x} \in f_{\lambda}^{-1}(U)$, the fiber $f_{\lambda}^{-1}\left(f_{\lambda}(\bar{x})\right)=X_{\lambda} \cap \bar{f}^{-1}(\bar{f}(\bar{x}))$ has dimension $\leq n$ at $\bar{x}$ since $\bar{x} \in W$. In other words, by replacing $\bar{X}$ with such an $X_{\lambda}$ we get to the situation in which all fibers of $\bar{f}^{-1}(U) \rightarrow U$ have dimension $\leq n$.

Let $\mathscr{M}=\mathscr{O}_{\bar{X}}$. Since $\bar{f}$ is finitely presented and the open set $V=\emptyset$ in $\bar{f}^{-1}(U)$ has complement whose fibers over $U$ have dimension $<n+1$, we can say that $\left.\mathscr{M}\right|_{\bar{f}^{-1}(U)}$ is $U$-flat in dimensions $\geq n+1$ (in the sense of [RG, I, 5.2.1]). Thus, by [RG, I, 5.7.9] there is a $U$-admissible blow-up $g: S^{\prime} \rightarrow S$ such that the strict 
transform $\bar{f}^{\prime}: \bar{X}^{\prime} \rightarrow S^{\prime}$ of $\bar{f}$ relative to $g$ is finitely presented over $S^{\prime}$ and there is an $S^{\prime}$-flat quasi-compact open $\bar{V}^{\prime} \subseteq \bar{X}^{\prime}$ such that $\operatorname{dim}\left(\left(\bar{X}^{\prime}-\bar{V}^{\prime}\right) / S^{\prime}\right) \leq n$ (that is, all fibers have dimension at most $n$ ). By [RG, I, 5.1.2(v), 5.1.4] (whose proofs also work for algebraic spaces instead of schemes), we can make a further $U$-admissible blow-up on $S^{\prime}$ to get to the situation in which $\bar{U}^{\prime}:=\bar{f}^{\prime-1}\left(U^{\prime}\right)$ is a schematically dense open subspace of $\bar{X}^{\prime}$, where $U^{\prime}$ denotes the preimage of $U$ in $S^{\prime}$. Note that $\operatorname{dim}\left(\bar{U}^{\prime} / S^{\prime}\right) \leq n$ since $S^{\prime} \rightarrow S$ is a $U$-admissible blow-up and $\operatorname{dim}\left(\bar{f}^{-1}(U) / U\right) \leq n$.

We claim that $\operatorname{dim}\left(\bar{X}^{\prime} / S^{\prime}\right) \leq n$, so since the strict transform $X^{\prime} \rightarrow S^{\prime}$ of $f$ relative to $g$ is a closed subspace of $\bar{X}^{\prime}$ over $S^{\prime}$ it would follow that $\operatorname{dim}\left(X^{\prime} / S^{\prime}\right) \leq n$, as desired. Since $\operatorname{dim}\left(\left(\bar{X}^{\prime}-\bar{V}^{\prime}\right) / S^{\prime}\right) \leq n$, we just have to check that $\operatorname{dim}\left(\bar{V}^{\prime} / S^{\prime}\right) \leq n$. The overlap $\bar{V}^{\prime} \cap \bar{U}^{\prime}$ is a schematically dense open subspace of $\bar{V}^{\prime}$ with fiber dimensions at most $n\left(\right.$ since $\left.\operatorname{dim}\left(\bar{U}^{\prime} / S^{\prime}\right) \leq n\right)$, so we may apply Lemma A.5.1 to conclude.

The proof of [RG, I, 5.7.11] begins with a finite type map $f: X \rightarrow S$ and asserts at the start (using $\mathrm{RG}$, I, 5.7.9]) that there exists a blow-up $S^{\prime} \rightarrow S$ (along a finite-type quasi-coherent ideal) with respect to which the strict transform of $X$ is flat and finitely presented over $S^{\prime}$. Since [RG, I, 5.7.9] has finite presentation hypotheses, an additional argument is needed to ensure the existence of such a blow-up when $f$ is of finite type rather than of finite presentation. We now prove this via a further application of Theorem 3.2.1.

Proposition A.5.3. Let $f: X \rightarrow S$ be a map of finite type between qcqs algebraic spaces, and let $U \subseteq S$ be a quasi-compact open subspace such that $f^{-1}(U) \rightarrow U$ is flat and finitely presented (e.g., an open immersion). There exists a $U$-admissible blow-up $g: S^{\prime} \rightarrow S$ such that the strict transform $f^{\prime}: X^{\prime} \rightarrow S^{\prime}$ of $f$ relative to $g$ is flat and finitely presented.

Proof. By Theorem 3.2.1, we may choose a closed immersion $i: X \hookrightarrow \bar{X}$ over $S$ into an algebraic space of finite presentation over $S$. Let $\bar{f}: \bar{X} \rightarrow S$ denote the structure map, and $\mathscr{M}=i_{*}\left(\mathscr{O}_{X}\right)$. Since $f^{-1}(U) \rightarrow U$ is finitely presented and flat, $\left.\mathscr{M}\right|_{\bar{f}^{-1}(U)}$ is finitely presented over $\mathscr{O}_{\bar{f}^{-1}(U)}$ and $U$-flat. In other words, $\left.\mathscr{M}\right|_{\bar{f}^{-1}(U)}$ is $U$-flat in dimension $\geq 0$ (in the sense of RG, I, 5.2.1]). Thus, by the finite presentedness of $\bar{f}$ we can apply [RG, I, 5.7.9] to construct a $U$-admissible blow-up $g: S^{\prime} \rightarrow S$ such that the strict transform $\bar{X}^{\prime} \rightarrow S^{\prime}$ of $\bar{f}$ relative to $g$ is finitely presented and the strict transform $\mathscr{M}^{\prime}$ of $\mathscr{M}$ is finitely presented over $\mathscr{O}_{\bar{X}^{\prime}}$ and $S^{\prime}$-flat. But $\mathscr{M}^{\prime}$ is the quotient of $\mathscr{O}_{\bar{X}^{\prime}}$ corresponding to the closed immersion $X^{\prime} \hookrightarrow \bar{X}^{\prime}$ of strict transforms relative to $g$. Hence, $X^{\prime}$ is $S^{\prime}$-flat and its closed immersion into $\bar{X}^{\prime}$ is finitely presented, so $X^{\prime} \rightarrow S^{\prime}$ is also finitely presented (as $\bar{X}^{\prime} \rightarrow S^{\prime}$ is finitely presented).

\section{REFERENCES}

[A] M. Artin, Algebraization of formal moduli : II. Existence of modifications, Annals of Math., 91 no. 1, 1970, pp. 88-135.

[C1] B. Conrad, Modular curves and rigid-analytic spaces, Pure and Applied Math Quarterly 2 no. 1, 2006, pp. 29-110.

[C2] B. Conrad, Deligne's notes on Nagata compactifications, Journal of the Ramanujan Math. Soc. 22 no. 3, 2007 , pp. 205-257.

[C3] B. Conrad, Keel-Mori theorem via stacks, unpublished notes at http://math.stanford.edu/ conrad.

[CdJ] B. Conrad, A. J. de Jong, Approximation of versal deformations, Journal of Algebra, 255 no. 2, 2002, pp. 489-515.

[D] P. Deligne, Le théorème de plongement de Nagata, personal notes (unpublished).

[SGA3] M. Demazure, A. Grothendieck, Schémas en groupes I, II, III, Lecture Notes in Math 151, 152, 153, Springer-Verlag, New York, 1970.

[EGA] A. Grothendieck, Élements de Géométrie Algébrique, Math. Publ. IHES 4, 8, 11, 17, 20, 24, 28, 32, $1960-7$.

[SGA1] A. Grothendieck, Revêtements étale et Groupe Fondamental, Lecture Notes in Mathematics 224, Springer-Verlag, New York, 1971.

[RG] L. Gruson, M. Raynaud, Critères de platitude et de projectivité, Inventiones Math. 13, 1971, pp. 1-89.

[I] L. Illusie, Complexe cotangent et déformations. I., Springer Lecture Notes 239, Springer-Verlag, New York, 1971.

[KM] S. Keel, S. Mori, Quotients by groupoids, Annals of Math. 145, 1997, pp. 193-213.

[K] D. Knutson, Algebraic spaces, Springer Lecture Notes 203, Springer-Verlag, New York, 1971.

[LMB] G. Laumon, L. Moret-Bailly, Champs algébriques, Ergebnisse der Mathematik 39, Springer-Verlag, Berlin, 2000.

[LO1] Y. Lazlo, M. Olsson, The six operations for sheaves on Artin stacks I, Publ. Math. IHES 107, 2008, pp. $109-168$.

[LO2] Y. Lazlo, M. Olsson, The six operations for sheaves on Artin stacks II, Publ. Math. IHES 107, 2008, pp. 169-210.

[L] W. Lütkebohmert, On compactification of schemes, Manuscripta Mathematica 80, 1993, pp. 95-111. 
[N1] M. Nagata, Imbedding of an abstract variety in a complete variety, J. Math. Kyoto 2 (1), 1962, pp. 1-10.

[N2] M. Nagata, A generalization of the imbedding problem, J. Math. Kyoto 3 (1), 1963, pp. 89-102.

[R1] J.-C. Raoult, Compactification des espaces algébriques normaux, C.R. Acad. Sci. Paris Sér. A-B 273, 1971, pp. A766A767.

[R2] J.-C. Raoult, Compactification des espaces algébriques, C.R. Acad. Sci. Paris Sér A-B 278, 1974, pp. 867-869.

[R3] J.-C. Raoult, Le théorème de compactification de Nagata pour les espaces algébriques, unpublished thesis, 1974.

[Ry1] D. Rydh, Existence of quotients by finite groups and coarse moduli spaces, arXiv:0708.3333 2007.

[Ry2] D. Rydh, Noetherian approximation of algebraic spaces and stacks, arXiv:0904.0227, 2009.

[TT] R. Thomason, T. Trobaugh, "Higher algebraic K-theory of schemes and of derived categories," in The Grothendieck Festschrift, Vol III, pp. 247-435, Progress in Mathematics, Birkhauser, Boston, 1990.

[Va] Y. Varshavsky, Lefschetz-Verdier trace formula and a generalization of a theorem of Fujiwara, Geom. Funct. Anal. 17, 2007, 271-319.

[Vo] P. Vojta, Nagata's embedding theorem, arXiv:0706.1907, 2007.

Department of Mathematics, Stanford University, Stanford, CA 48109, USA

E-mail address: conrad@math.stanford.edu

Department of Mathematics, University of Washington, Seattle, WA 98195 USA

E-mail address: lieblich@math.washington.edu

Department of Mathematics, Univ. of California, Berkeley, CA 94720, USA

E-mail address: molsson@math.berkeley.edu 